\title{
An excursion into geometric analysis
}

\author{
Tobias H. Colding and William P. Minicozzi II
}

\section{Contents}

1. Introduction 84

Part 1. Classical and almost classical results 86

2. Minimal surfaces $\quad 86$

3. The Bochner formula $\quad 88$

4. Monotonicity and the mean value inequality 90

5. The theorems of Bernstein and Bers 93

6. Mean curvature flow 94

7. Ricci flow $\quad 95$

8. Gradient estimates 96

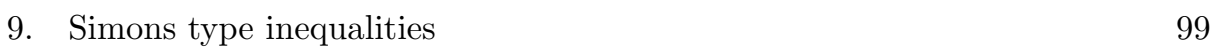

10. Minimal annuli with small total curvature are graphs 101

Part 2. The role of multi-valued graphs in minimal surfaces 105

$\begin{array}{ll}\text { 11. Basic properties of multi-valued graphs } & 105\end{array}$

12. Sharp estimates on the separation for multi-valued graphs 107

$\begin{array}{ll}\text { 13. Double-valued minimal graphs } & 109\end{array}$

14. Approximation by standard pieces 110

$\begin{array}{ll}\text { Part 3. Regularity theory } & 113\end{array}$

15. Hausdorff and Gromov-Hausdorff distances 114

$\begin{array}{ll}\text { 16. Reifenberg type conditions } & 114\end{array}$

$\begin{array}{ll}\text { 17. Monotonicity and regularity theory } & 115\end{array}$

18. Embedded minimal disks 118

19. Global theory of minimal surfaces in $\mathbf{R}^{3} \quad 123$

Part 4. Constructing minimal surfaces and applications 124

20. The Weierstrass representation $\quad 125$

21. Area-minimizing surfaces $\quad 126$

22. Index bounds for geodesics and minimal surfaces 127

23. The min-max construction of minimal surfaces 129

The authors were partially supported by NSF Grants DMS 0104453 and DMS 0104187. 
24. An application of min-max surfaces to Ricci flow

Part 5. Growth of harmonic functions

25. Harmonic functions and spherical harmonics 135

26. Manifolds with non-negative Ricci curvature 136

27. Minimal surfaces and a generalized Bernstein theorem 138

28. Volumes for eigensections 140

$\begin{array}{ll}\text { References } & 141\end{array}$

\section{Introduction}

This is a guided tour through some selected topics in geometric analysis. Most of the results here can be found in the literature but some are new and do not appear elsewhere. We have chosen to illustrate many of the basic ideas as they apply to the theory of minimal surfaces. This is, in part, because minimal surfaces is, if not the oldest, then certainly one of the oldest areas of geometric analysis dating back to Euler's work in the 1740's and in fact many of the basic ideas in geometric analysis originated in the study of minimal surfaces. In any case, the ideas apply to a variety of different fields and we will mention some of these as we go along.

Part 1 reviews some of the classical ideas and results in geometric analysis. We begin with the definition and basic results for minimal surfaces, including the first variation formula and maximum principle in Section 2. Section 3 gives some applications of the Bochner formula to comparison theorems, vanishing theorems (such as the famous Bochner theorem), and harmonic functions. We turn next to the monotonicity formula and mean value inequality in Section 4 . These play a fundamental role in many areas of geometric analysis, however, we have chosen to focus on the special cases of minimal surfaces and manifolds with non-negative Ricci curvature. Section 5 recalls the Bernstein theorem (for entire solutions) and Bers' theorem (for exterior solutions) of the minimal surface equation. These results illustrate an interesting rigidity for solutions of the minimal surface equation which comes from the nonlinearity (and does not occur for solutions of linear equations). In Sections 6 and 7, we briefly review the basic facts for mean curvature flow and Ricci flow. The next two sections discuss some fundamental a priori estimates in pde. First, Section 8 gives various gradient estimates for linear/nonlinear, elliptic/parabolic equations, all based on the maximum principle. The importance of this fundamental estimate has been well-understood since the work of Bernstein in the early 1900's. Section 9 recalls a much more recent tool for a priori estimates, namely, Simons' inequality, and illustrates its usefulness for proving a priori estimates. The original inequality of Simons was for the Laplacian of the norm squared of the second fundamental form of a minimal hypersurface, but variations of this inequality appear in a surprising number of fields (Einstein manifolds, harmonic maps, Yang-Mills connections, various parabolic equations, etc.). Finally, in Section 10 we derive the basic estimates for minimal annuli with small total curvature, including a quantitative form of Bers' theorem. This last section also sets the stage for some estimates in Part 2 for multi-valued graphs. 
In Part 2, we turn our attention to embedded multi-valued minimal graphs (the basic example is half of the helicoid). These are graphs of multi-valued functions and should be thought of as "spiral staircases." The analysis of these has played a major role in recent developments in minimal surface theory. The first two sections, 11 and 12, prove the fundamental estimates on the separation and curvature. Section 13 extends the Bers' theorem from Part 1 to this setting. Finally, Section 14 proves some original results, including a representation formula showing that an embedded multi-valued minimal graph can be written as a sum of a helicoid, a catenoid, and a small perturbation.

In Part 3, we survey some of the key ideas in classical regularity theory, recent developments on embedded minimal disks, and some global results for minimal surfaces in $\mathbf{R}^{3}$. Sections 15 and 16 focus on Reifenberg type conditions, where a set is assumed to be close to a plane at all points and at all scales ("close" is in the Hausdorff or Gromov-Hausdorff sense and is defined in Section 15). This condition automatically gives Hölder regularity (and hence higher regularity if the set is also a weak solution to a natural equation). Section 17 surveys the role of monotonicity and scaling in regularity theory, including $\epsilon$-regularity theorems (such as Allard's theorem) and tangent cone analysis (such as Almgren's refinement of Federer's dimension reducing). Section 18 briefly reviews recent results of the authors for embedded minimal disks, developing a regularity theory in a setting where the classical methods cannot be applied and in particular where there is no monotonicity. The estimates and ideas discussed in Section 18 have applications to the global theory of minimal surfaces in $\mathbf{R}^{3}$. In Section 19, we give a quick tour of some recent results in this classical, but rapidly developing, area.

Thus far, we have mainly dealt with regularity and a priori estimates but have ignored questions of existence. Part 4 surveys some of the most useful existence results for minimal surfaces and gives an application to Ricci flow. Section 20 recalls the classical Weierstrass representation, including a few modern applications, and the Kapouleas desingularization method. Section 21 deals with area minimizing surfaces (whether for fixed boundary, fixed homotopy class, etc.) and questions of embeddedness. The next section discusses unstable (hence not minimizing) surfaces and the corresponding questions for geodesics, concentrating on whether the Morse index can be bounded uniformly. Section 23 recalls the min-max construction for producing unstable minimal surfaces and, in particular, doing so while controlling the topology and guaranteeing embeddedness. Finally, Section 24 discusses a recent application of min-max surfaces to bound the extinction time for Ricci flow, answering a question of Perelman.

Finally, in Part 5, we discuss some global results for harmonic functions and a few applications of function theory. We begin by reviewing the basic theory of harmonic functions on Euclidean space. This starts with the Liouville theorem and the relationship between polynomial growth harmonic functions and eigenfunctions on the sphere; see Section 25. In Section 26 we sketch the proof that the spaces of harmonic functions of polynomial growth are finite dimensional on manifolds with non-negative Ricci curvature. Section 27 gives a version of this for minimal submanifolds and a geometric application of this. Finally, Section 28 discusses two estimates related to nodal sets of eigenfunctions. 


\section{Part 1. Classical and almost classical results}

\section{Minimal surfaces}

Let $\Sigma \subset \mathbf{R}^{3}$ be a smooth orientable surface (possibly with boundary) with unit normal $\mathrm{n}_{\Sigma}$. Given a function $\phi$ in the space $C_{0}^{\infty}(\Sigma)$ of infinitely differentiable (i.e., smooth), compactly supported functions on $\Sigma$, consider the one-parameter variation

$$
\Sigma_{t, \phi}=\left\{x+t \phi(x) \mathrm{n}_{\Sigma}(x) \mid x \in \Sigma\right\} .
$$

The so called first variation formula of area is the equation (integration is with respect to darea)

$$
\left.\frac{d}{d t}\right|_{t=0} \operatorname{Area}\left(\Sigma_{t, \phi}\right)=\int_{\Sigma} \phi H,
$$

where $H$ is the mean curvature of $\Sigma$. (When $\Sigma$ is noncompact, then $\Sigma_{t, \phi}$ in (2.2) is replaced by $\Gamma_{t, \phi}$, where $\Gamma$ is any compact set containing the support of $\phi$.) The surface $\Sigma$ is said to be a minimal surface (or just minimal) if

$$
\left.\frac{d}{d t}\right|_{t=0} \operatorname{Area}\left(\Sigma_{t, \phi}\right)=0 \quad \text { for all } \phi \in C_{0}^{\infty}(\Sigma)
$$

or, equivalently by $(2.2)$, if the mean curvature $H$ is identically zero. Thus $\Sigma$ is minimal if and only if it is a critical point for the area functional. (Since a critical point is not necessarily a minimum the term "minimal" is misleading, but it is time honored. The equation for a critical point is also sometimes called the Euler-Lagrange equation.) Moreover, a computation shows that if $\Sigma$ is minimal, then

$$
\left.\frac{d^{2}}{d t^{2}}\right|_{t=0} \operatorname{Area}\left(\Sigma_{t, \phi}\right)=-\int_{\Sigma} \phi L_{\Sigma} \phi
$$

where

$$
L_{\Sigma} \phi=\Delta_{\Sigma} \phi+|A|^{2} \phi
$$

is the second variational (or Jacobi) operator. Here $\Delta_{\Sigma}$ is the Laplacian on $\Sigma$ and $A$ is the second fundamental form. So $|A|^{2}=\kappa_{1}^{2}+\kappa_{2}^{2}$, where $\kappa_{1}, \kappa_{2}$ are the principal curvatures of $\Sigma$ and $H=\kappa_{1}+\kappa_{2}$. A minimal surface $\Sigma$ is said to be stable if

$$
\left.\frac{d^{2}}{d t^{2}}\right|_{t=0} \operatorname{Area}\left(\Sigma_{t, \phi}\right) \geq 0 \quad \text { for all } \phi \in C_{0}^{\infty}(\Sigma) \text {. }
$$

A graph (i.e., the set $\left.\left\{\left(x_{1}, x_{2}, u\left(x_{1}, x_{2}\right)\right) \mid\left(x_{1}, x_{2}\right) \in \Omega\right\}\right)$ of a real valued function $u$ on a domain $\Omega$ in $\mathbf{R}^{2}$ is minimal iff the function satisfies the minimal surface equation

$$
\operatorname{div}\left(\frac{d u}{\sqrt{1+|d u|^{2}}}\right)=0,
$$

where $d u$ is the $\mathbf{R}^{2}$ gradient of the function $u$ and div is the divergence in $\mathbf{R}^{2}$. One can show that a minimal graph is stable and, more generally, so is a multi-valued minimal graph (see below for the precise definition).

We will next derive the weak form of the minimal surface equation, i.e., the so-called first variation formula, which is the basic tool for working with "weak 
solutions" (typically, stationary varifolds). Let $X$ be a vector field on $\mathbf{R}^{3}$. We can write the divergence $\operatorname{div} \Sigma_{\Sigma} X$ of $X$ on $\Sigma$ as

$$
\operatorname{div}{ }_{\Sigma} X=\operatorname{div}_{\Sigma} X^{T}+X \cdot H
$$

where $X^{T}$ and $X^{N}$ are the tangential and normal projections of $X$. From this and Stokes' theorem, we see that $\Sigma$ is minimal if and only if for all vector fields $X$ with compact support and vanishing on the boundary of $\Sigma$,

$$
\int_{\Sigma} \operatorname{div} \Sigma_{\Sigma} X=0
$$

This equation is known as the first variation formula. It has the benefit that (2.9) makes sense as long as we can define the divergence on $\Sigma$. As a consequence of (2.9), we will show the following proposition:

Proposition 2.1. $\Sigma^{k} \subset \mathbf{R}^{n}$ is minimal if and only if the restrictions of the coordinate functions of $\mathbf{R}^{n}$ to $\Sigma$ are harmonic functions.

Proof. Let $\eta$ be a smooth function on $\Sigma$ with compact support and $\eta \mid \partial \Sigma=0$, then

$$
\int_{\Sigma}\left\langle\nabla_{\Sigma} \eta, \nabla_{\Sigma} x_{i}\right\rangle=\int_{\Sigma}\left\langle\nabla_{\Sigma} \eta, e_{i}\right\rangle=\int_{\Sigma} \operatorname{div} \Sigma\left(\eta e_{i}\right) .
$$

From this, the claim follows easily.

Recall that if $\Xi \subset \mathbf{R}^{n}$ is a compact subset, then the smallest convex set containing $\Xi$ (the convex hull, $\operatorname{Conv}(\Xi)$ ) is the intersection of all half-spaces containing $\Xi$. The maximum principle forces a minimal submanifold to lie in the convex hull of its boundary (this is the "convex hull property"):

Proposition 2.2. If $\Sigma^{k} \subset \mathbf{R}^{n}$ is a compact minimal submanifold, then $\Sigma \subset$ $\operatorname{Conv}(\partial \Sigma)$.

Proof. A half-space $H \subset \mathbf{R}^{n}$ can be written as

$$
H=\left\{x \in \mathbf{R}^{n} \mid\langle x, e\rangle \leq a\right\},
$$

for a vector $e \in \mathbf{S}^{n-1}$ and constant $a \in \mathbf{R}$. By Proposition 2.1, the function $u(x)=\langle e, x\rangle$ is harmonic on $\Sigma$ and hence attains its maximum on $\partial \Sigma$ by the maximum principle.

The argument in the proof of the convex hull property can be rephrased as saying that as we translate a hyperplane towards a minimal surface, the first point of contact must be on the boundary. When $\Sigma$ is a hypersurface, this is a special case of the strong maximum principle for minimal surfaces:

LEMMA 2.3. Let $\Omega \subset \mathbf{R}^{n-1}$ be an open connected neighborhood of the origin. If $u_{1}, u_{2}: \Omega \rightarrow \mathbf{R}$ are solutions of the minimal surface equation with $u_{1} \leq u_{2}$ and $u_{1}(0)=u_{2}(0)$, then $u_{1} \equiv u_{2}$.

See [CM1] for a proof of Lemma 2.3 and further discussion. 


\section{The Bochner formula}

On a Riemannian manifold $M$ a very useful formula of $\mathrm{S}$. Bochner asserts that for any function $u$ on $M$

$$
\frac{1}{2} \Delta|\nabla u|^{2}=\left|\operatorname{Hess}_{u}\right|^{2}+\langle\nabla \Delta u, \nabla u\rangle+\operatorname{Ric}_{M}(\nabla u, \nabla u) .
$$

Two special cases of this formula are particularly useful. When $u$ is a distance function, that is, when $|\nabla u|=1$, then the above formula reduces to

$$
0=|U|^{2}+\operatorname{Tr}\left(U^{\prime}\right)+\operatorname{Ric}_{M},
$$

where $U$ is the Hessian of $u$ and the Ricci curvature and the derivative $U^{\prime}$ is taken in the direction of the unit vector $\nabla u$. This is the so-called Ricatti equation. The other useful special case of the Bochner formula is when $u$ is a harmonic function. In this case, the Bochner formula reduces to

$$
\frac{1}{2} \Delta|\nabla u|^{2}=\left|\operatorname{Hess}_{u}\right|^{2}+\operatorname{Ric}_{M}(\nabla u, \nabla u) .
$$

So when $M$ has non-negative Ricci curvature, this formula implies that the energy density of $u$ is subharmonic.

The Laplacian and Hessian comparison theorems relate the distance function on $M$ to a space of constant curvature. These comparisons are essentially integrated forms of the Ricatti equation (3.2). For simplicity, we will not state the most general forms of these theorems, but rather only state the comparisons with $\mathbf{R}^{n}$.

The Laplacian comparison theorem compares $\Delta r$, where $r$ is the distance to a point, on $M$ with $\Delta|x|=(n-1) /|x|$ on Euclidean space:

THEOREM 3.1. If $M$ has non-negative Ricci curvature and $r$ is the distance function to a fixed point $p$, then

$$
\Delta r \leq \frac{n-1}{r}
$$

Moreover, (3.4) holds weakly even where $r$ is not smooth.

Proof. We will prove (3.4) assuming that $r$ is smooth so that $|\nabla r|=1$ (see $[\mathbf{C a}]$ for the extension to the general case). Let $\gamma$ be a geodesic from $p$ parametrized by arclength and set

$$
L(t)=\Delta r \circ \gamma(t) .
$$

Note that $L^{\prime}(t)=\langle\nabla \Delta r, \nabla r\rangle$ by the chain rule so that (3.2) gives

$$
L^{\prime}=\operatorname{Tr}\left(U^{\prime}\right) \leq-|U|^{2} \leq-L^{2} /(n-1) .
$$

Here the second inequality used the Cauchy-Schwarz inequality

$$
\left(\sum_{i=1}^{n-1} \lambda_{i}\right)^{2} \leq(n-1) \sum_{i=1}^{n-1} \lambda_{i}^{2}
$$

for the eigenvalues $\lambda_{i}$ of the matrix $U$ (there are at most $(n-1)$ non-zero eigenvalues since $U(\nabla r, \nabla r)=0)$. We can rewrite (3.6) as

$$
(1 / L)^{\prime} \geq 1 /(n-1) \text {. }
$$

(Notice that we get equality in $(3.8)$ for $L(t)=(n-1) / t$.) Since any manifold is "almost Euclidean" for $r$ small, it is easy to see that

$$
\lim _{r \rightarrow 0} r \Delta r=(n-1) \text {. }
$$


Integrating the differential equality (3.8) and substituting the "boundary condition" (3.9) gives

$$
\Delta r \circ \gamma(t)=L(t) \leq \frac{n-1}{t} .
$$

Since $\gamma$ was arbitrary, the theorem follows.

We note two immediate consequences of Theorem 3.1:

- Since $\nabla r$ is the unit normal to the geodesic spheres, the mean curvature of these spheres is at most $(n-1) / r$.

- The square of the distance function satisfies

$$
\Delta r^{2}=2|\nabla r|^{2}+2 r \Delta r \leq 2 n .
$$

The Hessian comparison theorem is somewhat more restrictive since it requires bounds on the sectional curvatures of $M$; of course, the conclusion is correspondingly stronger. The following theorem is a useful special case of the Hessian comparison theorem:

THEOREM 3.2. If $M$ is simply connected with non-positive sectional curvature and $r$ is the distance function to a fixed point $p$, then

$$
\operatorname{Hess}_{r}(X, X) \geq \frac{|X-\langle X, \nabla r\rangle \nabla r|^{2}}{r}
$$

for any vector $X$.

An important application of (3.1) (and similar formulas) is to prove vanishing theorems relating a pointwise curvature condition to global properties of $M$. The prototype is the Bochner theorem (see also $[\mathbf{C 1}],[\mathbf{C 2}]$ for an extension of this famous theorem of Bochner that had been conjectured by M. Gromov):

TheOREM 3.3. [Bc] If $M^{n}$ is closed with Ric $M \geq 0$, then each harmonic 1-form is parallel. In particular, the space of harmonic 1-forms is at most n-dimensional.

Proof. (Sketch) A harmonic 1-form $\alpha$ can be written locally as $d u$ where $u$ is a (locally defined) harmonic function. In particular, (3.1) implies that

$$
\Delta|\alpha|^{2}=\Delta|\nabla u|^{2} \geq 2\left|\operatorname{Hess}_{u}\right|^{2}=2|\nabla \alpha|^{2} .
$$

Since $M$ is closed (in particular, $\partial M=\emptyset$ ), Stokes' theorem gives

$$
0=\int \Delta|\alpha|^{2} \geq 2 \int|\nabla \alpha|^{2}
$$

Therefore, $|\nabla \alpha|^{2}$ vanishes identically.

Therefore, by the Hodge theorem, the first betti number of a closed manifold $M$ with non-negative Ricci curvature is at most $n$ with equality only if the universal cover of $M$ is $\mathbf{R}^{n}$. There have been many geometric applications of this method, where analytic methods (like the Hodge theorem) use topology to produce solutions of a pde and then a curvature condition (like the Bochner formula) places restrictions on these solutions.

Finally, we note that (3.1) can be used to prove an eigenvalue comparison theorem when $M$ has positive Ricci curvature. Namely, A. Lichnerowicz showed that if $\operatorname{Ric}_{M} \geq \operatorname{Ric}_{\mathbf{S}^{n}}$, then the first (non-zero) eigenvalue $\lambda_{1}(M) \geq \lambda_{1}\left(\mathbf{S}^{n}\right)$ : 
TheOrem 3.4. [Lc] If $M^{n}$ is closed with Ric $M \geq(n-1)$, then $\lambda_{1}(M) \geq n$.

Proof. Let $u$ be a (non-constant) eigenfunction on $M$ with $\Delta u=-\lambda u$. We will show that $\lambda \geq n$. After normalizing so $\int u^{2}=1$, Stokes' theorem gives

$$
\int|\nabla u|^{2}=-\int u \Delta u=\lambda \int u^{2}=\lambda \text {. }
$$

Substituting the equation for $u$ into the Bochner formula (3.1) gives

$$
\frac{1}{2} \Delta|\nabla u|^{2} \geq\left|\operatorname{Hess}_{u}\right|^{2}+(n-1-\lambda)|\nabla u|^{2} \geq \frac{\lambda^{2}}{n} u^{2}+(n-1-\lambda)|\nabla u|^{2},
$$

where the last inequality used the Cauchy-Schwarz inequality as in (3.7). Integrating (3.16) over $M$ gives

$$
0=\frac{1}{2} \int \Delta|\nabla u|^{2} \geq \frac{\lambda^{2}}{n} \int u^{2}+(n-1-\lambda) \int|\nabla u|^{2}=\lambda \frac{n-1}{n}(n-\lambda) .
$$

Remark 3.5. These comparison theorems are sharp in the sense that equality is achieved on the model spaces. The converse of this is also true, i.e., equality is achieved only for the model spaces, and is known as "rigidity." For example, if $M^{n}$ is closed with $\operatorname{Ric}_{M} \geq(n-1)$ and $\lambda_{1}(M)=n$, then M. Obata, [Ob], proved that $M=\mathbf{S}^{n}$. It is then natural to ask how stable is this rigidity - i.e., what happens if equality is almost achieved? These questions, known as "almost rigidity," were answered by Colding and J. Cheeger-Colding; see [C2], [ChC4] and references therein. Moreover, almost rigidity theorems have played a key role in regularity theory; see [ChC1], [ChC2], [ChC3].

\section{Monotonicity and the mean value inequality}

Monotonicity formulas and mean value inequalities play a fundamental role in many areas of geometric analysis. In this section, we focus on the specific cases of minimal surfaces and manifolds with non-negative Ricci curvature.

Before we state and prove the monotonicity formula of volume for minimal submanifolds, we will need to recall the coarea formula. This formula asserts (see, for instance, $[\mathbf{F e}]$ for a proof) that if $\Sigma$ is a manifold and $h: \Sigma \rightarrow \mathbf{R}$ is a proper (i.e., $h^{-1}((-\infty, t])$ is compact for all $\left.t \in \mathbf{R}\right)$ Lipschitz function on $\Sigma$, then for all locally integrable functions $f$ on $\Sigma$ and $t \in \mathbf{R}$

$$
\int_{\{h \leq t\}} f|\nabla h|=\int_{-\infty}^{t} \int_{h=\tau} f d \tau .
$$

Proposition 4.1. Suppose that $\Sigma^{k} \subset \mathbf{R}^{n}$ is a minimal submanifold and $x_{0} \in$ $\mathbf{R}^{n}$; then for all $0<s<t$

$$
t^{-k} \operatorname{Vol}\left(B_{t}\left(x_{0}\right) \cap \Sigma\right)-s^{-k} \operatorname{Vol}\left(B_{s} \cap \Sigma\right)=\int_{\left(B_{t}\left(x_{0}\right) \backslash B_{s}\left(x_{0}\right)\right) \cap \Sigma} \frac{\left|\left(x-x_{0}\right)^{N}\right|^{2}}{\left|x-x_{0}\right|^{k+2}} .
$$

Proof. Within this proof, we set $B_{t}=B_{t}\left(x_{0}\right)$. Since $\Sigma$ is minimal,

$$
\Delta_{\Sigma}\left|x-x_{0}\right|^{2}=2 \operatorname{div} \Sigma_{\Sigma}\left(x-x_{0}\right)=2 k .
$$

By Stokes' theorem integrating this gives

$$
2 k \operatorname{Vol}\left(B_{s} \cap \Sigma\right)=\int_{B_{s} \cap \Sigma} \Delta_{\Sigma}\left|x-x_{0}\right|^{2}=2 \int_{\partial B_{s} \cap \Sigma}\left|\left(x-x_{0}\right)^{T}\right| .
$$


Using this and the coarea formula (i.e., (4.1)), an easy calculation gives

$$
\begin{aligned}
\frac{d}{d s}\left(s^{-k} \operatorname{Vol}\left(B_{s} \cap \Sigma\right)\right) & =-k s^{-k-1} \operatorname{Vol}\left(B_{s} \cap \Sigma\right)+s^{-k} \int_{\partial B_{s} \cap \Sigma} \frac{\left|x-x_{0}\right|}{\left|\left(x-x_{0}\right)^{T}\right|} \\
& =s^{-k-1} \int_{\partial B_{s} \cap \Sigma}\left(\frac{\left|x-x_{0}\right|^{2}}{\left|\left(x-x_{0}\right)^{T}\right|}-\left|\left(x-x_{0}\right)^{T}\right|\right) \\
& =s^{-k-1} \int_{\partial B_{s} \cap \Sigma} \frac{\left|\left(x-x_{0}\right)^{N}\right|^{2}}{\left|\left(x-x_{0}\right)^{T}\right|} .
\end{aligned}
$$

Integrating and applying the coarea formula once more gives the claim.

Notice that $\left(x-x_{0}\right)^{N}$ vanishes precisely when $\Sigma$ is conical about $x_{0}$, i.e., when $\Sigma$ is invariant under dilations about $x_{0}$. As a corollary, we get the following:

Corollary 4.2. Suppose that $\Sigma^{k} \subset \mathbf{R}^{n}$ is a minimal submanifold and $x_{0} \in$ $\mathbf{R}^{n}$; then the function

$$
\Theta_{x_{0}}(s)=\frac{\operatorname{Vol}\left(B_{s}\left(x_{0}\right) \cap \Sigma\right)}{\operatorname{Vol}\left(B_{s} \subset \mathbf{R}^{k}\right)}
$$

is a nondecreasing function of $s$. Moreover, $\Theta_{x_{0}}(s)$ is constant in $s$ if and only if $\Sigma$ is conical about $x_{0}$.

Of course, if $x_{0}$ is a smooth point of $\Sigma$, then $\lim _{s \rightarrow 0} \Theta_{x_{0}}(s)=1$; the Allard regularity theorem gives the converse of this.

The monotonicity of area is an very useful tool in the regularity theory for minimal surfaces - at least when there is some a priori area bound. For instance, this monotonicity and a compactness argument allow one to reduce many regularity questions to questions about minimal cones (this was a key observation of W. Fleming in his work on the Bernstein problem; see Section 5). Similar monotonicity formulas have played key roles in other geometric problems, including harmonic maps, Yang-Mills connections, J-holomorphic curves, and regularity of limit spaces with a lower Ricci curvature bound.

Arguing as in Proposition 4.1, we get a weighted monotonicity:

Proposition 4.3. If $\Sigma^{k} \subset \mathbf{R}^{n}$ is a minimal submanifold, $x_{0} \in \mathbf{R}^{n}$, and $f$ is a function on $\Sigma$, then

$=\int_{\left(B_{t}\left(x_{0}\right) \backslash B_{s}\left(x_{0}\right)\right) \cap \Sigma} f \frac{\left|\left(x-x_{0}\right)^{N}\right|^{2}}{\left|x-x_{0}\right|^{k+2}}+\frac{1}{2} \int_{s}^{t} \tau^{-k-1} \int_{B_{\tau}\left(x_{0}\right) \cap \Sigma}\left(\tau^{2}-\left|x-x_{0}\right|^{2}\right) \Delta_{\Sigma} f d \tau$.

We get immediately the following mean value inequality for the special case of non-negative subharmonic functions:

Corollary 4.4. Suppose that $\Sigma^{k} \subset \mathbf{R}^{n}$ is a minimal submanifold, $x_{0} \in \mathbf{R}^{n}$, and $f$ is a non-negative subharmonic function on $\Sigma$; then

$$
s^{-k} \int_{B_{s}\left(x_{0}\right) \cap \Sigma} f
$$

is a nondecreasing function of $s$. In particular, if $x_{0} \in \Sigma$, then for all $s>0$

$$
f\left(x_{0}\right) \leq \frac{\int_{B_{s}\left(x_{0}\right) \cap \Sigma} f}{\operatorname{Vol}\left(B_{s} \subset \mathbf{R}^{k}\right)} .
$$


Another interesting (and crucial) appearance of monotonicity is the volume comparison theorem of Bishop-Gromov for manifolds with non-negative Ricci curvature, $[\mathbf{G L P a}]$; see also $[\mathbf{P e 1}]$ for a generalization to Ricci flow. In the case of non-negative Ricci curvature, the monotonicity goes the opposite direction:

THEOREM 4.5. If a $k$-dimensional manifold $M$ has non-negative Ricci curvature, then

$$
\frac{\operatorname{Vol}\left(B_{s}\left(x_{0}\right) \subset M\right)}{\operatorname{Vol}\left(B_{s} \subset \mathbf{R}^{k}\right)}
$$

is a non-increasing function of $s$.

Proof. (Sketch) By the Laplacian comparison theorem, we have

$$
\Delta_{M} r^{2} \leq 2 k
$$

where $r$ is the distance function to $x_{0}$. Integrating this by parts gives

$$
2 k \operatorname{Vol}\left(B_{s}\left(x_{0}\right)\right) \geq \int_{B_{s}\left(x_{0}\right)} \Delta r^{2}=2 s \int_{\partial B_{s}\left(x_{0}\right)}|\nabla r|=2 s \frac{d}{d s} \operatorname{Vol}\left(B_{s}\left(x_{0}\right)\right),
$$

where the last equality used the co-area formula (since $|\nabla r|=1$ almost everywhere). This differential inequality gives (4.10).

Remark 4.6. Equation (4.10) immediately implies a volume doubling property for manifolds with non-negative Ricci curvature:

$$
\operatorname{Vol}\left(B_{2 s}\left(x_{0}\right) \subset M\right) \leq 2^{n} \operatorname{Vol}\left(B_{s}\left(x_{0}\right) \subset M\right) .
$$

This property is very useful for covering arguments, cf. [G].

We conclude this section with a well-known intrinsic mean value inequality which is often useful but difficult to find in the literature (it is often stated only for subharmonic functions).

Proposition 4.7. There exists $C=C(k)$ so that if $M$ is $k$-dimensional, Ric $_{M} \geq-(k-1) s^{-2}$, and $u \geq 0$ satisfies $\Delta_{M} u \geq-s^{-2} u$, then

$$
u^{2}(x) \leq \frac{C}{\operatorname{Vol}\left(B_{s}(x)\right)} \int_{B_{s}(x)} u^{2} .
$$

Proof. After rescaling the metric by $s$, it suffices to prove the case $s=1$. Let $N=M \times[-1,1]$ have the product metric, so that $\operatorname{Ric}_{N} \geq-(k-1)$. Define an auxiliary function $w$ on $N$ by

$$
w(x, t)=u(x) \mathrm{e}^{t}
$$

An easy calculation shows that

$$
\Delta_{N} w=\mathrm{e}^{t} \Delta_{M} u+\mathrm{e}^{t} u \geq 0
$$

so that $w$ is subharmonic. The mean value inequality for subharmonic functions (see theorem 6.2 on page 77 of [ScYa1]) then gives

$$
\begin{aligned}
w^{2}(x, 0) & \leq \frac{C}{\operatorname{Vol}\left(B_{1}(x, 0) \subset N\right)} \int_{B_{1}(x, 0) \subset N} w^{2} \\
& \leq 2 \mathrm{e}^{2} \frac{C}{\operatorname{Vol}\left(B_{1 / 2}(x) \subset M\right)} \int_{B_{1}(x) \subset M} u^{2} .
\end{aligned}
$$


The proposition follows from this after we use the Bishop-Gromov volume comparison (cf. Theorem 4.5) to bound

$$
\frac{\operatorname{Vol}\left(B_{1}(x)\right)}{\operatorname{Vol}\left(B_{1 / 2}(x)\right)} .
$$

\section{The theorems of Bernstein and Bers}

A classical theorem of S. Bernstein from 1916 says that entire (i.e., defined over all of $\mathbf{R}^{2}$ ) minimal graphs are planes. This remarkable theorem of Bernstein was one of the first illustrations of the fact that the solutions to a nonlinear PDE, like the minimal surface equation, can behave quite differently from the solutions to a linear equation. Rather surprisingly, this result very much depended on the dimension. The combined efforts of S. Bernstein $[\mathbf{B e}]$, E. De Giorgi $[\mathbf{D G}]$, F. J. Almgren, Jr. [Am1], and J. Simons [Sim] finally gave:

TheOREM 5.1. If $u: \mathbf{R}^{n-1} \rightarrow \mathbf{R}$ is an entire solution to the minimal surface equation and $n \leq 8$, then $u$ is an affine function.

However, in 1969 E. Bombieri, De Giorgi, and E. Giusti [BDGG] constructed entire non-affine solutions to the minimal surface equation on $\mathbf{R}^{8}$ and an areaminimizing singular cone in $\mathbf{R}^{8}$. In fact, they showed that for $m \geq 4$ the cones

$$
C_{m}=\left\{\left(x_{1}, \ldots, x_{2 m}\right) \mid x_{1}^{2}+\cdots+x_{m}^{2}=x_{m+1}^{2}+\cdots+x_{2 m}^{2}\right\} \subset \mathbf{R}^{2 m}
$$

are area-minimizing (and obviously singular at the origin).

One way to prove the Bernstein theorem is to prove a curvature estimate for minimal graphs. The basic example is the estimate of E. Heinz for surfaces:

TheOREM 5.2. [He] If $D_{r_{0}} \subset \mathbf{R}^{2}$ and $u: D_{r_{0}} \rightarrow \mathbf{R}$ satisfies the minimal surface equation, then for $\Sigma=G_{\text {raph }}$ and $0<\sigma \leq r_{0}$

$$
\sigma^{2} \sup _{D_{r_{0}-\sigma}}|A|^{2} \leq C \text {. }
$$

The original Bernstein Theorem follows from Theorem 5.2 by taking $r_{0} \rightarrow \infty$. By the same reasoning, the examples of [BDGG] show that (5.2) cannot hold for all dimensions. However, curvature estimates for graphs over $B_{r_{0}} \subset \mathbf{R}^{n-1}$ were proven in [ScSiYa] for $n \leq 6$ (the remaining cases, i.e., $n=7$ and 8 , were proven in $[\mathbf{S i 2}])$.

In contrast to the entire case, exterior solutions of the minimal graph equation, i.e., solutions on $\mathbf{R}^{2} \backslash B_{1}$, are much more plentiful. In this case, Theorem 5.2 only gives quadratic curvature decay $|A|^{2} \leq C|x|^{-2}$. In particular, it is not even clear that $|\nabla u|$ is bounded since

$$
|\nabla| \nabla u|| \leq C|x|^{-1}
$$

is not integrable along rays. However, L. Bers proved that $\nabla u$ actually has an asymptotic limit:

THEOREM 5.3. [Ber] If $u$ is a $C^{2}$ solution to the minimal surface equation on $\mathbf{R}^{2} \backslash B_{1}$, then $\nabla u$ has a limit at infinity (i.e., there is an asymptotic tangent plane).

Bers' theorem was extended to higher dimensions by L. Simon: 
THEOREM 5.4. [Si1] If $u$ is a $C^{2}$ solution to the minimal surface equation on $\mathbf{R}^{n} \backslash B_{1}$, then either

- $|\nabla u|$ is bounded and $\nabla u$ has a limit at infinity.

- All tangent cones at infinity are of the form $\Sigma \times \mathbf{R}$ where $\Sigma$ is singular.

Bernstein's theorem has had many other interesting generalizations, including, e.g., curvature estimates of R. Schoen for stable surfaces and Schoen-Simon-Yau for stable hypersurfaces with bounded density. In the early nineteen-eighties Schoen and Simon extended the theorem of Bernstein to complete simply connected embedded minimal surfaces in $\mathbf{R}^{3}$ with quadratic area growth. A surface $\Sigma$ is said to have quadratic area growth if for all $r>0$, the intersection of the surface with the ball in $\mathbf{R}^{3}$ of radius $r$ and center at the origin is bounded by $C r^{2}$ for a fixed constant $C$ independent of $r$. In corollary 1.18 in [CM4], this was generalized to quadratic area growth for intrinsic balls:

Corollary 5.5. [CM4] Given a constant $C_{I}$, there exists $C_{P}$ so that if $\mathcal{B}_{2 s} \subset$ $\Sigma \subset \mathbf{R}^{3}$ is an embedded minimal disk with

$$
\int_{\mathcal{B}_{2 s}}|A|^{2} \leq C_{I}
$$

then

$$
\sup _{\mathcal{B}_{s}}|A|^{2} \leq C_{P} s^{-2}
$$

\section{Mean curvature flow}

Just as the Laplace equation has the heat equation as a parabolic analog, mean curvature flow is the parabolic analog of the minimal surface equation. A oneparameter family of smooth hypersurfaces $\left\{M_{t}\right\} \subset \mathbf{R}^{n+1}$ flows by mean curvature if

$$
z_{t}=\mathbf{H}(z)=\Delta_{M_{t}} z,
$$

where $z$ are coordinates on $\mathbf{R}^{n+1}$ and $\mathbf{H}=-H \mathrm{n}$ is the mean curvature vector.

Example 6.1. Let $M_{t}$ be the family of concentric shrinking $n$-spheres of radius

$$
\sqrt{R^{2}-2 n t}
$$

It is easy to see that $M_{t}$ flows by mean curvature and is smooth up to $t=R^{2} /(2 n)$ when it shrinks to a point.

Suppose now that each $M_{t}$ is the graph of a function $u(\cdot, t)$. So, if $z=(x, y)$ with $x \in \mathbf{R}^{n}$, then $M_{t}$ is given by $y=u(x, t)$ which satisfies

$$
u_{t}=\left(1+|d u|^{2}\right)^{1 / 2} \operatorname{div}\left(\frac{d u}{\left(1+|d u|^{2}\right)^{1 / 2}}\right),
$$

where $d u$ is the $\mathbf{R}^{n}$ gradient of the function $u$ and div is divergence in $\mathbf{R}^{n}$.

The monotonicity formula and mean value inequality of Section 4 have analogs in mean curvature flow. The monotonicity formula, proven by G. Huisken (and extended to more general weak solutions by T. Ilmanen and B. White), is:

THEOREM 6.2. [H] If a smooth one-parameter family of hypersurfaces $M_{t}$ flows by mean curvature in $\mathbf{R}^{n+1} \times[-T, 0]$, then

$$
\frac{d}{d t} \int_{M_{t}}(-4 \pi t)^{-n / 2} e^{\frac{|x|^{2}}{4 t}}=-\int_{M_{t}}\left|\mathbf{H}-\frac{x^{N}}{2 t}\right|^{2}(-4 \pi t)^{-n / 2} e^{\frac{|x|^{2}}{4 t}} .
$$


In particular, the "density ratio"

$$
\int_{M_{t}}(-4 \pi t)^{-n / 2} e^{\frac{|x|^{2}}{4 t}}
$$

is non-increasing.

The restrictions of the coordinate functions to $M_{t}$ satisfy the heat equation

$$
\partial_{t} x_{i}=\Delta_{M} x_{i} .
$$

From this, we see that the restriction of $|x|^{2}$ satisfies

$$
\left(\partial_{t}-\Delta_{M}\right)|x|^{2}=-2 n .
$$

As in the stationary case (i.e., for minimal surfaces), this is the key to the proof of Theorem 6.2.

The mean value inequality in this case applies to non-negative solutions of the heat equation on $M_{t}$; we refer to [E1] for more detail on this as well as discussion of the local monotonicity formula for mean curvature flow proven in [E2].

The parabolic maximum principle has been very useful in mean curvature flow (somewhat similarly to the convex hull property for minimal surfaces). Two immediate, but useful, consequences are:

1. Disjoint surfaces stay disjoint.

2. An embedded surface stays embedded (as long as it evolves smoothly).

The reason for (1) and (2) is quite simple. Suppose that two initially disjoint surfaces touch at a first time $t$ at a point $x$. Clearly, they will be tangent at $(x, t)$ so nearby we see two graphs, one above the other. Hence, at $x$ the mean curvature of the upper graph is larger (or equal to). Therefore, the upper graph crossed over the lower at a slightly early time, contradicting that $t$ is first time of contact.

Combining (1) with the shrinking spheres of Example 6.1, we see that any compact hypersurface flowing by mean curvature has a finite extinction time.

\section{Ricci flow}

The Ricci flow is the parabolic analog of the Einstein equation $\operatorname{Ric}_{M}=$ Constant $g$, where $g$ is the metric. Namely, let $M^{3}$ be a fixed smooth manifold and let $g(t)$ be a one-parameter family of metrics on $M$ evolving by the Ricci flow, so

$$
\partial_{t} g=-2 \operatorname{Ric}_{M_{t}} .
$$

Short-time existence for the Ricci flow was established by Hamilton:

THEOREM 7.1. [Ha2] Given any smooth compact Riemannian manifold $\left(M, g_{0}\right)$, there exists a unique smooth solution $g(t)$ to (7.1) with initial condition $g(0)=g_{0}$ on some time interval $[0, \epsilon)$.

Long-time existence is quite a bit more subtle, see [Ha2] and [Pe1].

There are many formal similarities between the Ricci flow and the mean curvature flow, including similarities between the evolution equations for various geometric quantities. One interesting distinction is the evolution equation for the scalar curvature $R=R(t)$ under the Ricci flow (see, for instance, page 16 of [Ha3])

$$
\partial_{t} R=\Delta R+2|\mathrm{Ric}|^{2} \geq \Delta R+\frac{2}{n} R^{2}
$$


where the inequality used the Cauchy-Schwarz inequality ( $M$ is $n$-dimensional). This differential inequality has an interesting consequence: After flowing for any positive amount of time, there is a lower bound for the scalar curvature (the mean curvature flow has no such analog). To make this precise, a straightforward maximum principle argument gives that at time $t>0$

$$
R(t) \geq \frac{1}{1 /[\min R(0)]-2 t / n}=-\frac{n}{2(t+C)} .
$$

In the derivation of $(7.3)$ we implicitly assumed that $\min R(0)<0$. If this was not the case, then (7.3) trivially holds with $C=0$, since, by $(7.2), \min R(t)$ is always non-decreasing.

\section{Gradient estimates}

Gradient estimates have played a key role in geometry and pde since at least the early work of Bernstein. These are probably the most fundamental a priori estimates for elliptic and parabolic equations, leading to Harnack inequalities, Liouville theorems, and compactness theorems for both linear and nonlinear pde.

A typical example for linear equations is the well-known gradient estimate of S.Y. Cheng and S.T. Yau for harmonic functions:

THEOREM 8.1. [CgYa] If $\Delta u=0$ on $B_{r}(0)$ with non-negative Ricci curvature, then

$$
|\nabla u|(0) \leq C r^{-1}\|u\|_{\infty},
$$

where $\|u\|_{\infty}$ is the sup norm of the function $u$ on $B_{r}(0)$.

To give something of the flavor, we will use the maximum principle to prove 8.1 on the Euclidean unit ball $B_{1}(0) \subset \mathbf{R}^{n}$.

Proof. (of Theorem 8.1 for $B_{1}(0) \subset \mathbf{R}^{n}$.) Define the cutoff function

$$
\eta(x)=1-|x|^{2},
$$

so that $|\nabla \eta| \leq 2$ and $\Delta \eta=-2 n$. We compute that

$$
\begin{aligned}
\Delta\left(\eta^{2}|\nabla u|^{2}\right) & \geq-2 n|\nabla u|^{2}-16 \eta|\nabla u|\left|\operatorname{Hess}_{u}\right|+2 \eta^{2}\left|\operatorname{Hess}_{u}\right|^{2} \\
& \geq-(2 n+32)|\nabla u|^{2},
\end{aligned}
$$

where the last inequality used the absorbing inequality

$$
16 a b \leq 2 a^{2}+32 b^{2} .
$$

In particular, the function

$$
w=(n+16) u^{2}+\eta^{2}|\nabla u|^{2}
$$

is subharmonic on $B_{1}(0)$ (i.e., $\Delta w \geq 0$ ). By the maximum principle, the maximum of $w$ occurs on the boundary so that

$$
|\nabla u|^{2}(0) \leq w(0) \leq \max _{\partial B_{1}(0)} w=(n+16) \max _{\partial B_{1}(0)} u^{2} .
$$


In fact, Cheng and Yau prove a stronger estimate: If in addition $u$ is positive on $B_{r}(0)$, then

$$
|\nabla \log u|(0) \leq C r^{-1} .
$$

An important consequence is the Harnack inequality for positive harmonic functions Elliptic Harnack inequality:

$$
\sup _{B_{r / 2}(0)} u \leq C^{\prime} \inf _{B_{r / 2}(0)} u \text {. }
$$

Proof. Suppose the sup and inf are achieved at $p, q \in \partial B_{r / 2}(0)$. Fix a curve $\gamma_{p, q} \subset B_{r / 2}(0)$ from $p$ to $q$ of length at most $r$ (e.g., connect each point to 0 by a ray). Integrating the bound

$$
\sup _{B_{r / 2}(0)}|\nabla \log u| \leq 2 C r^{-1}
$$

over $\gamma_{p, q}$ gives

$$
\log \frac{u(p)}{u(q)} \leq \int_{\gamma_{p, q}}|\nabla \log u| \leq 2 C
$$

This gradient estimate also gives the global Liouville theorem of Yau, [Ya3]: Liouville theorem: If $u$ is a positive harmonic function on a complete manifold with non-negative Ricci curvature, then $u$ is constant.

Proof. We can take $r \rightarrow \infty$ in (8.7) to get that $|\nabla u|=0$.

The parabolic analog of Theorem 8.1 is the gradient estimate for the heat equation of $\mathrm{P}$. Li and Yau (we will state the version for $M$ complete):

THEOREM 8.2. [LiYa] If $u$ is a positive solution of $\partial_{t} u=\Delta u$ for $0 \leq t$ on a complete $M$ with non-negative Ricci curvature, then

$$
2 t\left(|\nabla \log u|^{2}-\partial_{t} \log u\right) \leq n,
$$

or, equivalently, $2 t \Delta \log u \geq-n$.

ProOF. (Sketch) Set

$$
w=-t \Delta \log u=t\left(|\nabla \log u|^{2}-\partial_{t} \log u\right) .
$$

The key calculation is (cf. lemma 1 on page 155 of [ScYa1])

$$
t\left(\Delta-\partial_{t}\right) w \geq \frac{2}{n} w^{2}-w-2 t \nabla w \cdot \nabla \log u .
$$

Suppose that $w$ achieves its maximum on $M \times[0, t]$ at $(x, t)$ (for example, when $M$ is compact; otherwise we use a cutoff). The parabolic maximum principle then gives $\nabla w(x, t)=0$ and $\left(\Delta-\partial_{t}\right) w(x, t) \leq 0$. Substituting this into (8.13) gives

$$
0 \geq \frac{2}{n} w^{2}(x, t)-w(x, t),
$$

so that $w(x, t) \leq n / 2$ as desired. 
Integrating this along curves as in the elliptic case gives for $t_{1}<t_{2}$ that Parabolic Harnack inequality:

$$
u\left(x_{1}, t_{1}\right) \leq u\left(x_{2}, t_{2}\right)\left(\frac{t_{2}}{t_{1}}\right)^{\frac{n}{2}} \mathrm{e}^{\frac{\mathrm{dist}^{2}\left(x_{1}, x_{2}\right)}{4\left(t_{2}-t_{1}\right)}} .
$$

In [Ha1], R. Hamilton gave an extension of (8.11) to a full matrix estimate whose trace was (8.11). For example, if $u$ is positive solution of the heat equation on $\mathbf{R}^{n} \times[0, T]$, then $[\mathbf{H a 1}]$ implies that

$$
2 t \operatorname{HesS}_{\log u}+\delta_{i j} \geq 0 .
$$

Taking the trace of (8.16) gives $|\nabla \log u|^{2}-\partial_{t} \log u \leq n /(2 t)$.

8.1. Gradient estimates for nonlinear equations. For the (nonlinear) minimal surface equation, the situation is somewhat different. In this case, i.e., when the graph of $u$ is minimal on $B_{r}(0)$, then Bombieri, De Giorgi, and M. Miranda proved in $[\mathbf{B D M}]$ that

$$
\log |d u|(0) \leq C\left(1+r^{-1}\|u\|_{\infty}\right),
$$

where $d u$ is the $\mathbf{R}^{n}$ gradient of the function $u$ (the case of surfaces was done by $\mathrm{R}$. Finn in $[\mathbf{F i}])$. By an earlier example of Finn, this exponential dependence cannot be improved. In $[\mathbf{K}]$, N. Korevaar gave a maximum principle proof of a weaker form of $[\mathbf{B D M}]$; this weaker form had $\|u\|_{\infty}^{2}$ in place of $\|u\|_{\infty}$.

In [CM12], we proved a sharp gradient estimate for graphs flowing by mean curvature:

TheOrem 8.3. [CM12] There exists $C=C(n)$ so if the graph of

$$
u: B_{\sqrt{2 n+1} r} \times\left[0, r^{2}\right] \rightarrow \mathbf{R}
$$

flows by mean curvature, then

$$
\log |d u|\left(0, r^{2} /[4 n]\right) \leq C\left(1+r^{-1}\|u(\cdot, 0)\|_{\infty}\right)^{2} .
$$

The quadratic dependence on $\|u(\cdot, 0)\|_{\infty}$ in (8.19) should be compared with the linear dependence which holds when the graph of $u$ is minimal (i.e., $u_{t}=0$ ). Somewhat surprisingly, examples in [CM12] show that this quadratic dependence on $\|u(\cdot, 0)\|_{\infty}$ is sharp.

The first gradient estimate for mean curvature flow was proven by Ecker and Huisken who adapted Korevaar's argument to mean curvature flow in theorem 2.3 of $[\mathbf{E H 2}]$ to get

(8.20) $\log |d u|\left(0, r^{2} /[4 n]\right) \leq 1 / 2 \log \left(1+\|d u(\cdot, 0)\|_{\infty}^{2}\right)+C\left(1+r^{-1}\|u(\cdot, 0)\|_{\infty}\right)^{2}$.

Note that, unlike (8.19), the gradient bound (8.20) depends also on the initial bound for the gradient.

8.2. Generalizations. The Harnack inequality actually holds for much more general spaces. For instance, L. Saloff-Coste and A. Grigor'yan (see $[\mathbf{S C}]$ and $[\mathbf{G r}]$ ) have shown that the following two properties suffice

Volume doubling: There exists $C_{D}$ so that

$$
\operatorname{Vol}\left(B_{2 r}(x)\right) \leq C_{D} \operatorname{Vol}\left(B_{r}(x)\right),
$$

for all $r>0$ and points $x$. 
Neumann Poincaré inequality: There exists $C_{N}$ so that if $\int_{B_{r}(x)} f=0$, then

$$
\int_{B_{r}(x)} f^{2} \leq C_{N} r^{2} \int_{B_{r}(x)}|\nabla f|^{2},
$$

for all $r>0$ and points $x$.

These properties, however, do not imply the gradient estimate. Note that manifolds with non-negative Ricci curvature satisfy both conditions (the Poincaré inequality essentially follows from $[\mathbf{B u}]$, cf. also $[\mathbf{J e}]$ ).

The De Giorgi, Nash, Moser theory (see chapter 8 in [GiTr] or section 4.4 in [HnLn]) gives a Harnack inequality as long as we have a volume doubling and a Sobolev inequality. The difference between a Sobolev and Poincaré inequality is that a Sobolev controls an $L^{p}$-norm of $f$ for some $p>2$. Surprisingly, in [HzKo], P. Hajlasz and P. Koskela showed that the volume doubling and Neumann Poincaré inequality together imply a Sobolev inequality, thereby recovering the above result of Saloff-Coste and Grigor'yan. See $[\mathbf{C h C 2}],[\mathbf{C h C 3}],[\mathbf{H j}]$ for more such "low regularity" analysis (including analysis on singular spaces).

\section{Simons type inequalities}

In this section, we recall a very useful differential inequality for the Laplacian of the norm squared of the second fundamental form of a minimal hypersurface $\Sigma$ in $\mathbf{R}^{n}$ and illustrate its role in a priori estimates. This inequality, originally due to J. Simons (see [CM1] for a proof and further discussion), is:

LEMMA 9.1. [Sim] If $\Sigma^{n-1} \subset \mathbf{R}^{n}$ is a minimal hypersurface, then

$$
\Delta_{\Sigma}|A|^{2}=-2|A|^{4}+2\left|\nabla_{\Sigma} A\right|^{2} \geq-2|A|^{4} .
$$

An inequality of the type (9.1) on its own does not lead to pointwise bounds on $|A|^{2}$ because of the nonlinearity. However, it does lead to estimates if a "scaleinvariant energy" is small. For example, H. Choi and Schoen used (9.1) to prove:

TheOREM 9.2. [CiSc] If $0 \in \Sigma \subset B_{r}(0)$ with $\partial \Sigma \subset \partial B_{r}(0)$ is a minimal surface with sufficiently small total curvature $\int|A|^{2}$, then

$$
|A|^{2}(0) \leq r^{-2} \text {. }
$$

Analogs of (9.1) occur in a surprising number of geometric problems. For example, when $u: M^{m} \rightarrow N^{n}$ is a harmonic map, the energy density $|d u|^{2}$ satisfies this type of inequality, leading to an a priori estimate when $u$ has small scaleinvariant energy

$$
r^{2-m} \int_{B_{r}}|d u|^{2}
$$

(see $[\mathbf{S a U h}],[\mathbf{S c 2}]$ ). Similar inequalities hold for the curvature of a Yang-Mills connection or the curvature of an Einstein manifold. When $M$ is an Einstein manifold, its curvature tensor $R$ satisfies (see $[\mathbf{H a 2}]$ or equation (2.6) in $[\mathbf{A n 1}$ ]; $[\mathbf{H a 2}]$ also establishes a parabolic analog for the Ricci flow)

$$
\Delta_{M}|R| \geq-C|R|^{2} .
$$

We next use (9.4) to prove an estimate for Einstein manifolds (cf. lemma 2.1 in [An1]). For simplicity, we restrict to the case $\operatorname{Ric}_{M}=0$. 
THEOREM 9.3. There exist $\epsilon=\epsilon(n)>0$, such that if $M^{n}$ is an $n$-dimensional Ricci-flat (Einstein) manifold and for some $x \in M$ either

$$
\operatorname{Vol}\left(B_{r}(x)\right) \geq[1-\epsilon] \operatorname{Vol}\left(B_{r} \subset \mathbf{R}^{n}\right)
$$

or

$$
\int_{B_{r}(x)}|R|^{n / 2}<\epsilon r^{-n} \operatorname{Vol}\left(B_{r}(x)\right)
$$

then $|R|(x) \leq C r^{-2}$.

Proof. We will prove that (9.6) gives the pointwise curvature bound; the other case is similar. Set

$$
F(z)=\left(r-4 \operatorname{dist}_{M}(x, z)\right)|R|^{1 / 2}(z),
$$

so that

$$
F(x)=r|R|^{1 / 2}(x) \geq 0, \text { and }\left.F\right|_{B_{r}(x) \backslash B_{r / 4}(x)} \leq 0 .
$$

Therefore, it suffices to prove that $F \leq C$ for some fixed constant $C$.

We will assume that $\max _{B_{r}(x)} F>32$ and deduce a contradiction if $\epsilon>0$ is sufficiently small. Let $y$ be a point where the maximum of $F$ is achieved and set $s=|R|^{-1 / 2}(y)$. Since $F(y)>32$, we have $32 s<\left|r-4 \operatorname{dist}_{M}(x, y)\right|$ so that for $z \in B_{s}(y)$

$$
1 / 2 \leq \frac{\left|r-4 \operatorname{dist}_{M}(x, y)\right|}{\left|r-4 \operatorname{dist}_{M}(x, z)\right|} \leq 2 .
$$

Since $F(z) \leq F(y)$, it follows that $B_{s}(y)$ satisfies

$$
\sup _{B_{s}(y)}|R|^{1 / 2} \leq 2|R|^{1 / 2}(y)=2 / s,
$$

so that (9.4) gives on $B_{s}(y)$ that

$$
\Delta_{M}|R| \geq-C|R|^{2} \geq-4 C s^{-2}|R| .
$$

Furthermore, the Bishop-Gromov volume comparison, i.e., Theorem 4.5, gives

$$
\frac{\operatorname{Vol}\left(B_{s}(y)\right)}{s^{n}} \geq \frac{\operatorname{Vol}\left(B_{r / 2}(y)\right)}{(r / 2)^{n}} \geq 2^{-n} \frac{\operatorname{Vol}\left(B_{r}(x)\right)}{r^{n}} .
$$

It follows from this and (9.6) that $B_{s}(y)$ also satisfies

$$
\int_{B_{s}(y)}|R|^{n / 2}<2^{n} \epsilon \frac{\operatorname{Vol}\left(B_{s}(y)\right)}{s^{n}} .
$$

Using (9.10), (9.11), and (9.13), the mean value inequality (Proposition 4.7) gives

$$
s^{-n}=|R|^{n / 2}(y) \leq \frac{C}{\operatorname{Vol}\left(B_{s}(y)\right)} \int_{B_{s}(y)}|R|^{n / 2}<C 2^{n} \epsilon s^{-n} .
$$

This gives a contradiction for $\epsilon$ sufficiently small.

Remark 9.4. We could alternatively have proven Theorem 9.3 by integral methods, i.e., using Moser iteration. However, the above proof by scaling is both shorter and more elementary. 
Finally, we mention that (9.1) has a parabolic version as well (see proposition 2.15 in [E1]): If $M_{t}$ flows by mean curvature, then

$$
\left(\frac{\partial}{\partial t}-\Delta_{M_{t}}\right)|A|^{2}=2|A|^{4}-2\left|\nabla_{M_{t}} A\right|^{2}
$$

As in the elliptic case, this Simons' type inequality is a crucial ingredient for establishing curvature estimates.

\section{Minimal annuli with small total curvature are graphs}

It is easy to see that a minimal disk with small total curvature must be a graph away from its boundary:

If $\int_{B_{R} \cap \Sigma}|A|^{2}<\epsilon$, then Theorem 9.2 gives

$$
\sup _{B_{R / 2} \cap \Sigma}|A|^{2}<C \epsilon / R^{2} .
$$

Integrating this (since $|\nabla \mathrm{n}| \leq|A|$ ) implies that each component of $B_{R / 2} \cap \Sigma$ is a graph if $\epsilon>0$ is small enough.

However, the corresponding question for minimal annuli is more subtle. We shall discuss this and some related problems in this section.

In [CM10], we gave three proofs that a minimal annulus with small total curvature is a graph. The first used a singular integral formula which had previously been useful for estimating nodal and singular sets; see Proposition 10.1 below and compare $[\mathbf{D o}]$. The second, and easiest, applies more generally to surfaces with quasi-conformal Gauss maps; see Proposition 10.2. The third, which is outlined in Lemma 10.3, was the one which could be extended to "annuli with slits" — i.e., embedded double-valued minimal graphs.

In this section, $\Sigma \subset \mathbf{R}^{3}$ is a compact connected oriented immersed surface. If $a \in \mathbf{S}^{2}, a^{\perp}$ denotes $\left\{x \in \mathbf{R}^{3} \mid\langle x, a\rangle=0\right\}$. For $a, b \in \mathbf{S}^{2}$, Angle $(a, b)$ is the angle between $a^{\perp}, b^{\perp}$; i.e.,

$$
\operatorname{Angle}(a, b)=\operatorname{dist}_{\mathbf{S}^{2}}(a,\{b,-b\}) .
$$

Let $f$ be harmonic on $\Sigma^{2}$ with critical points $\left\{y_{i}\right\}$ with multiplicities $\left\{m_{i}\right\}$. Suppose that none of the $y_{i}$ 's lie on $\partial \Sigma$. The Bochner formula on $\Sigma \backslash\left\{y_{i}\right\}$ gives

$$
\Delta_{\Sigma} \log \left|\nabla_{\Sigma} f\right|^{2}=2 \frac{\left|\operatorname{Hess}_{f}\right|^{2}}{\left|\nabla_{\Sigma} f\right|^{2}}+2 K-\frac{\left.\left.\left|\nabla_{\Sigma}\right| \nabla_{\Sigma} f\right|^{2}\right|^{2}}{\left|\nabla_{\Sigma} f\right|^{4}}=2 K .
$$

Here we used that since $\Delta_{\Sigma} f=0$ and $\Sigma$ is 2-dimensional, then

$$
2\left|\operatorname{Hess}_{f}\right|^{2}\left|\nabla_{\Sigma} f\right|^{2}=\left.\left.\left|\nabla_{\Sigma}\right| \nabla_{\Sigma} f\right|^{2}\right|^{2} .
$$

Hence, by Stokes' theorem

$$
\begin{aligned}
\int_{\partial \Sigma} \frac{d \log \left|\nabla_{\Sigma} f\right|^{2}}{d n} & =\int_{\Sigma \backslash\left\{y_{i}\right\}} \Delta_{\Sigma} \log \left|\nabla_{\Sigma} f\right|^{2}+4 \pi \sum_{i} m_{i} \\
& =2 \int_{\Sigma} K+4 \pi \sum_{i} m_{i} .
\end{aligned}
$$

Proposition 10.1. [CM10] If $\Sigma$ is connected and minimal with boundaries $\sigma_{1}$ and $\sigma_{2}, \int_{\sigma_{1} \cup \sigma_{2}}|A|<\pi / 8$, and $\int_{\Sigma} K \geq-\pi$, then $\Sigma$ is graphical. 
Proof. Fix $q_{i} \in \sigma_{i}$. Since

$$
\left|\nabla_{\Sigma} \operatorname{dist}_{\mathbf{S}^{2}}\left(\mathrm{n}\left(q_{i}\right), \mathrm{n}(\cdot)\right)\right| \leq|A|,
$$

the assumption on $\partial \Sigma$ gives

$$
\sum_{i} \sup _{z_{i} \in \sigma_{i}} \operatorname{dist}_{\mathbf{S}^{2}}\left(\mathrm{n}\left(q_{i}\right), \mathrm{n}\left(z_{i}\right)\right) \leq \sum_{i} \int_{\sigma_{i}}|A|<\pi / 8 .
$$

Choose $b \in \mathbf{S}^{2}$ with $\operatorname{Angle}\left(\mathrm{n}\left(q_{i}\right), b\right) \leq \pi / 4$ for $i=1,2$. We will show that $\Sigma$ is graphical over the plane $b^{\perp}$. By the triangle inequality and (10.7), for $i=1,2$,

$$
\sup _{z_{i} \in \sigma_{i}} \operatorname{Angle}\left(b, \mathrm{n}\left(z_{i}\right)\right) \leq \pi / 4+\sup _{z_{i} \in \sigma_{i}} \operatorname{dist}_{\mathbf{S}^{2}}\left(\mathrm{n}\left(q_{i}\right), \mathrm{n}\left(z_{i}\right)\right)<3 \pi / 8 .
$$

Rotate coordinates so that $b=(0,0,1)$ and $b^{\perp}$ is the $x_{1}-x_{2}-$ plane. Fix $\theta$ and set

$$
f=x_{1} \cos \theta+x_{2} \sin \theta .
$$

Given $x \in \Sigma$,

$$
\left|\nabla_{\Sigma} f\right|^{2}(x)=1-\langle(\cos \theta, \sin \theta, 0), \mathrm{n}(x)\rangle^{2} \geq\langle b, \mathrm{n}(x)\rangle^{2} .
$$

On $\partial \Sigma=\sigma_{1} \cup \sigma_{2},(10.8)$ and (10.10) imply that

$$
\inf _{\partial \Sigma}\left|\nabla_{\Sigma} f\right| \geq \inf _{\partial \Sigma}|\langle b, \mathrm{n}(x)\rangle|>\cos (3 \pi / 8)>1 / 3 .
$$

Since $\left|\nabla_{\Sigma}\right| \nabla_{\Sigma} f|| \leq\left|\operatorname{Hess}_{f}\right| \leq|A|,(10.11)$ gives on $\partial \Sigma$

$$
\left.\left|\nabla_{\Sigma} \log \right| \nabla_{\Sigma} f\right|^{2}\left|=\frac{2\left|\nabla_{\Sigma}\right| \nabla_{\Sigma} f||}{\left|\nabla_{\Sigma} f\right|} \leq 6\right| A \mid
$$

Integrating (10.12), we get

$$
\int_{\partial \Sigma}\left|\frac{d \log \left|\nabla_{\Sigma} f\right|^{2}}{d n}\right| \leq 6 \int_{\partial \Sigma}|A|<3 \pi / 4 .
$$

Since $\Sigma$ is minimal, $\Delta_{\Sigma} f=0$. Substituting (10.13) into (10.5),

$$
4 \pi \sum_{i} m_{i}=\int_{\partial \Sigma} \frac{d \log \left|\nabla_{\Sigma} f\right|^{2}}{d n}-2 \int_{\Sigma} K<3 \pi / 4+2 \pi<4 \pi ;
$$

hence, $f$ has no critical points. Since this is true for any $\theta, \Sigma$ is graphical over $b^{\perp}$.

In fact, Proposition 10.1 holds for $\Sigma$ whose Gauss map n is quasi-conformal:

Proposition 10.2. [CM10] If $\Sigma \subset \mathbf{R}^{3}$ is connected, $\int_{\Sigma}|K| \leq \pi,|A|^{2} \leq$ $C|K|$, and $\partial \Sigma$ has components $\left\{\sigma_{i}\right\}_{1 \leq i \leq n}$ with

$$
\sum_{i=1}^{n} \inf _{a \in \mathbf{S}^{2}} \sup _{x \in \sigma_{i}}\left\{\operatorname{dist}_{\mathbf{S}^{2}}(n(x), a)\right\}<\epsilon<\pi / 8,
$$

then $n(\Sigma) \subset \mathcal{B}_{2 \epsilon}(a)$ for some $a \in \mathbf{S}^{2}$ and $\Sigma$ is the graph of $u$ over $a^{\perp}$ with $|\nabla u| \leq 4 \epsilon$. 
10.1. Holomorphic functions on annuli. We next estimate the oscillation of a holomorphic function with small gradient on annuli. Since the Gauss map of a minimal annulus is conformal and its derivative is the second fundamental form $A$, this serves as a good model (in fact, the proof can easily be adapted to that case).

Suppose that $f$ is holomorphic on an annulus $D_{R} \backslash D_{\delta}$ and satisfies

$$
|\nabla f| \leq \epsilon /(2 \pi|z|) .
$$

Integrating this around each circle, we see that the oscillation of $f$ on each circle is at most $\epsilon$. However, if we simply integrate this bound radially to compare $f$ on the different circles, we get the log of the ratio of the radii. The next lemma improves on this to get a bound independent of this ratio; the key is to keep track of cancellation rather than integrating the bound on $|\nabla f|$.

LEMMA 10.3. If $f: D_{R} \backslash D_{\delta} \rightarrow \mathbf{C}$ is holomorphic and

$$
\int_{\partial D_{R} \cup \partial D_{\delta}}|\nabla f| \leq \epsilon
$$

then

$$
\min _{c \in \mathbf{C}} \max _{z}|f(z)-c| \leq \epsilon .
$$

Proof. For $\delta \leq s \leq R$, define the circular average of $f$ by

$$
I(s)=(2 \pi s)^{-1} \int_{\partial D_{s}} f=(2 \pi)^{-1} \int_{0}^{2 \pi} f\left(s \mathrm{e}^{i \theta}\right) d \theta,
$$

and $c=I(\delta)$. Differentiating (10.19), we have

$$
\begin{aligned}
2 \pi s I^{\prime}(s) & =\int_{\partial D_{s}} \frac{\partial f}{\partial r}=-i s^{-1} \int_{\partial D_{s}} \frac{\partial f}{\partial \theta}=-i \int_{0}^{2 \pi} \frac{\partial f}{\partial \theta} d \theta \\
& =-i\left[f\left(s \mathrm{e}^{i 2 \pi}\right)-f\left(s \mathrm{e}^{0}\right)\right]=0,
\end{aligned}
$$

where we used that

$$
\frac{\partial f}{\partial r}=-i r^{-1} \frac{\partial f}{\partial \theta}
$$

since $f$ is holomorphic. In particular, $I(R)=c$. Since $I(s)$ is the average of $f$ over $\partial D_{s}$, there exist $y_{1}, y_{2} \in \partial D_{R}$ with

$$
c=\operatorname{Re}\left(f\left(y_{1}\right)\right)+i \operatorname{Im}\left(f\left(y_{2}\right)\right) .
$$

Combining (10.22) with $\int_{\partial D_{R} \cup \partial D_{\delta}}|\nabla f| \leq \epsilon$,

$$
\max _{y \in \partial D_{R}}|\operatorname{Re}(f(y)-c)| \leq \epsilon / 2 \text { and } \max _{y \in \partial D_{R}}|\operatorname{Im}(f(y)-c)| \leq \epsilon / 2,
$$

so that $|f-c| \leq \epsilon$ on $\partial\left(D_{R} \backslash D_{\delta}\right)$. The maximum principle then gives (10.18).

Note that Lemma 10.3 does not hold for harmonic functions (in particular, (10.20) does not hold); e.g., take $\epsilon \log r /(4 \pi)$ and $R>\mathrm{e}^{8 \pi} \delta$. 
10.2. Bers' Theorem revisited. We have seen that an annulus with small total curvature must be graphical, even as the outer radius goes to infinity. However, Bers' Theorem indicates that much more should be true: the unit normal actually goes to a limit at infinity. This follows from a sharper decay estimate for the curvature. To keep things simple, we will give the argument for a holomorphic function with small gradient as in the previous lemma.

Lemma 10.4. If $f: D_{R} \backslash D_{1} \rightarrow \mathbf{C}$ is holomorphic and $|\nabla f(z)| \leq 1 /|z|$, then

$$
\int_{D_{R^{1 / 2} e^{t}} \backslash D_{R^{1 / 2} e^{-t}}}|\nabla f|^{2} \leq 2 \pi e^{2 t} / R .
$$
Set

Proof. By (10.20), we can subtract a constant so that $\int_{\partial D_{r}} f=0$ for all $r$.

$$
E(t)=\int_{D_{R^{1 / 2} \mathrm{e}^{t}} \backslash D_{R^{1 / 2} \mathrm{e}^{-t}}}|\nabla f|^{2} .
$$

Since $f$ is holomorphic, the Cauchy-Riemann equations give

$$
d \bar{f} \wedge d f=i|\nabla f|^{2} d x \wedge d y .
$$

Applying Stokes' theorem, we get

$$
E(t)=-i \int_{D_{R^{1 / 2} \mathrm{e}^{t}} \backslash D_{R^{1 / 2} \mathrm{e}^{-t}}} d \bar{f} \wedge d f=-i \int_{\partial\left(D_{R^{1 / 2} \mathrm{e}^{t}} \backslash D_{R^{1 / 2} \mathrm{e}^{-t}}\right)} \bar{f} d f .
$$

Differentiating (10.25), the chain rule and $|\nabla f| \leq 1 /|z|$ give

$$
E^{\prime}(t)=R^{1 / 2} \mathrm{e}^{-t} \int_{\partial D_{R^{1 / 2} \mathrm{e}^{-t}}}|\nabla f|^{2}+R^{1 / 2} \mathrm{e}^{t} \int_{\partial D_{R^{1 / 2} \mathrm{e}^{t}}}|\nabla f|^{2} \leq 4 \pi .
$$

The Cauchy-Schwarz and Wirtinger inequalities (recall that $\int_{\partial D_{r}} f=0$ ) give

$$
\begin{aligned}
\left|\int_{\partial D_{r}} \bar{f} d f\right| & =\left|\int_{\partial D_{r}} \bar{f} f_{\theta} d \theta\right| \leq\left(\int_{\partial D_{r}}|\bar{f}|^{2} d \theta\right)^{1 / 2}\left(\int_{\partial D_{r}}\left|f_{\theta}\right|^{2} d \theta\right)^{1 / 2} \\
& \leq \int_{\partial D_{r}}\left|f_{\theta}\right|^{2} d \theta=r / 2 \int_{\partial D_{r}}|\nabla f|^{2}
\end{aligned}
$$

where the last equality used the Cauchy-Riemann equations to relate $f_{\theta}$ and $f_{r}$. By (10.27)-(10.29), we get

$$
2 E(t) \leq E^{\prime}(t)
$$

and $E(1 / 2 \log R) \leq 2 \pi$. Integrating the differential inequality (10.30) yields (10.24).

Combining Lemma 10.4 (with $t=\log 2$ ) and the mean value inequality, we get

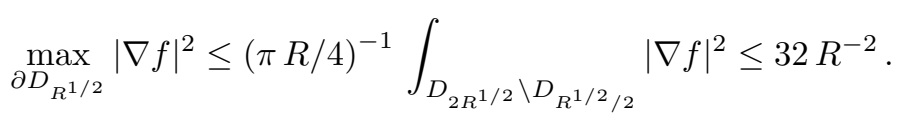

If we now take $R \rightarrow \infty$ as in Bers' theorem, then we see that

$$
|\nabla f(z)| \leq C|z|^{-2} \text {. }
$$

The bound (10.32) is integrable radially (as $|z| \rightarrow \infty$ ), so we see that $f$ has an asymptotic limit.

Finally, note that $f(z)=1 / z$ shows that (10.31) is sharp (up to the constant). 


\section{Part 2. The role of multi-valued graphs in minimal surfaces}

There are two local models for embedded minimal disks (by an embedded disk we mean a smooth injective map from the closed unit ball in $\mathbf{R}^{2}$ into $\mathbf{R}^{3}$ ). One model is the plane (or, more generally, a minimal graph) and the other is a piece of a helicoid.

The second model comes from the helicoid which was discovered by Meusnier in 1776. Meusnier had been a student of Monge. He also discovered that the surface now known as the catenoid is minimal in the sense of Lagrange, and he was the first to characterize a minimal surface as a surface with vanishing mean curvature. Unlike the helicoid, the catenoid is not topologically a plane but rather a cylinder.

The helicoid is a "double spiral staircase" (see [CM17]):

Example 2: (Helicoid; see fig. 1). The helicoid is the minimal surface in $\mathbf{R}^{3}$ given by the parametrization

$$
(s \cos t, s \sin t, t), \quad \text { where } s, t \in \mathbf{R} .
$$

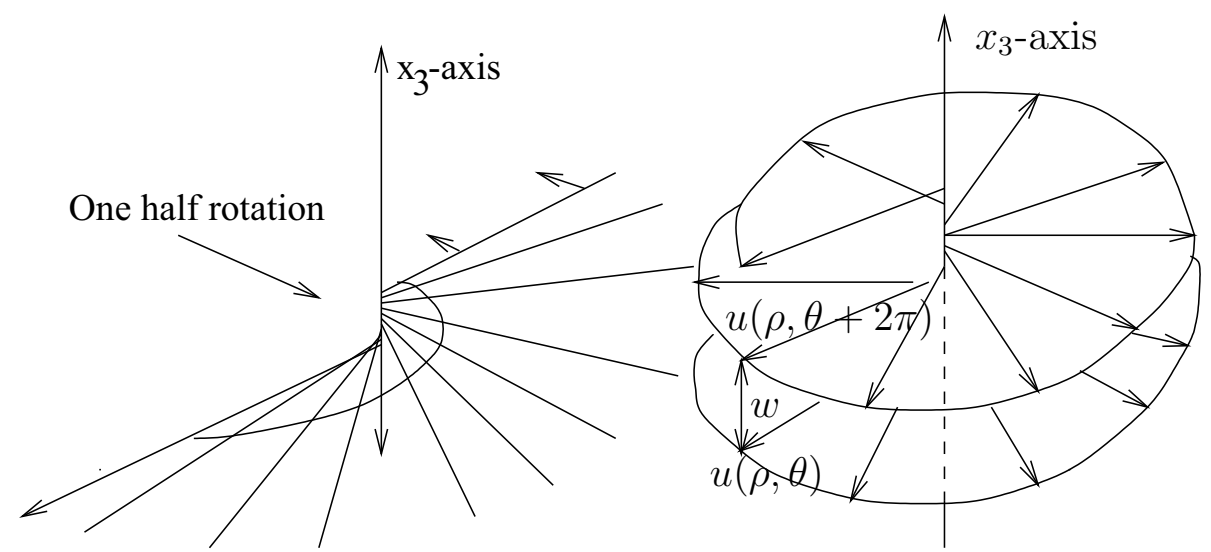

Figure 1. Multi-valued graphs. The helicoid is obtained by gluing together two $\infty$-valued graphs along a line.
Figure 2. The separation $w$ grows/decays in $\rho$ at most sublinearly for a multi-valued minimal graph; see (11.6).

\section{Basic properties of multi-valued graphs}

We will need the notion of a multi-valued graph, each staircase will be a multivalued graph. Intuitively, an (embedded) multi-valued graph is a surface such that over each point of the annulus, the surface consists of $N$ graphs. To make this notion precise, let $D_{r}$ be the disk in the plane centered at the origin and of radius $r$ and let $\mathcal{P}$ be the universal cover of the punctured plane $\mathbf{C} \backslash\{0\}$ with global polar coordinates $(\rho, \theta)$ so $\rho>0$ and $\theta \in \mathbf{R}$. An $N$-valued graph on the annulus $D_{s} \backslash D_{r}$ is a single valued graph of a function $u$ over

$$
\{(\rho, \theta)|r<\rho \leq s,| \theta \mid \leq N \pi\} .
$$

For working purposes, we generally think of the intuitive picture of a multi-sheeted surface in $\mathbf{R}^{3}$, and we identify the single-valued graph over the universal cover with its multi-valued image in $\mathbf{R}^{3}$. 
The multi-valued graphs that we will consider will all be embedded, which corresponds to a nonvanishing separation between the sheets (or the floors). Here the separation is the function (see fig. 2)

$$
w(\rho, \theta)=u(\rho, \theta+2 \pi)-u(\rho, \theta) .
$$

If $\Sigma$ is the helicoid, then $\Sigma \backslash\left\{x_{3}\right.$ - axis $\}=\Sigma_{1} \cup \Sigma_{2}$, where $\Sigma_{1}, \Sigma_{2}$ are $\infty$-valued graphs on $\mathbf{C} \backslash\{0\} . \Sigma_{1}$ is the graph of the function $u_{1}(\rho, \theta)=\theta$ and $\Sigma_{2}$ is the graph of the function $u_{2}(\rho, \theta)=\theta+\pi$. ( $\Sigma_{1}$ is the subset where $s>0$ in (10.33) and $\Sigma_{2}$ the subset where $s<0$.) In either case the separation $w=2 \pi$. A multi-valued minimal graph is a multi-valued graph of a function $u$ satisfying the minimal surface equation.

Note that for an embedded multi-valued graph, the sign of $w$ determines whether the multi-valued graph spirals in a left-handed or right-handed manner, in other words, whether upwards motion corresponds to turning in a clockwise direction or in a counterclockwise direction.

11.1. The sublinear growth of the separation. As we have seen, the separation is constant for the multi-valued graphs coming from each half of the helicoid. This can be viewed as a type of Liouville Theorem reflecting the conformal properties of an infinite-valued graph. In Proposition II.2.12 of [CM3], we proved a corresponding gradient estimate:

Proposition 11.1. [CM3] Given $\alpha>0$, there exists $N_{g}$ so that if $u$ satisfies the minimal surface equation on

$$
\left\{e^{-N_{g}} R \leq \rho \leq e^{N_{g}} R,-N_{g} \leq \theta \leq 2 \pi+N_{g}\right\},
$$

$|\nabla u| \leq 1$, and has separation $w \neq 0$, then

$$
\left|\operatorname{Hess}_{u}\right|(R, 0)+|\nabla \log | w||(R, 0) \leq \alpha / R .
$$

One important consequence of (11.4) is that, for $\alpha<1$, the separation grows sublinearly:

Corollary 11.2. Given $\alpha>0$, there exists $N_{g}$ so that if $u$ satisfies the minimal surface equation on

$$
\left\{e^{-N_{g}} r_{1} \leq \rho \leq e^{N_{g}} r_{2},-N_{g} \leq \theta \leq 2 \pi+N_{g}\right\},
$$

$|\nabla u| \leq 1$, and has separation $w \neq 0$, then

$$
|w|\left(r_{2}, 0\right) \leq|w|\left(r_{1}, 0\right)\left(\frac{r_{2}}{r_{1}}\right)^{\alpha} .
$$

Proof. Integrate $|\nabla \log | w|| \leq \alpha / \rho$ along the ray $\theta=0$ to get

$$
\log \frac{w\left(r_{2}, 0\right)}{w\left(r_{1}, 0\right)} \leq \alpha \int_{r_{1}}^{r_{2}} \rho^{-1} d \rho=\log \left(\frac{r_{2}}{r_{1}}\right)^{\alpha} .
$$

Since $u(\cdot, \cdot)$ and its $2 \pi$-rotation $u(\cdot, \cdot+2 \pi)$ are both solutions of the minimal surface equation, the difference $w$ is almost a solution of the linearized equation (which is the Jacobi equation in this case). Since the graphs have bounded gradient, this equation is not too far from the Laplace equation. To give some indication of why (11.4) holds, we will give an elementary proof when $u$ and $w$ are harmonic. 
Proof. (of Proposition 11.1 when $u$ is harmonic.) After rescaling, we can assume that $R=1$. By making the conformal change of coordinates

$$
(\rho, \theta) \rightarrow(\log \rho, \theta)
$$

we get a positive harmonic function

$$
\tilde{w}(x, y)=w\left(\mathrm{e}^{x}, y\right)
$$

defined on the square $\left[-N_{g}, N_{g}\right] \times\left[-N_{g}, N_{g}\right]$. Since the chain rule gives

$$
\nabla \log w(1,0)=\nabla \log \tilde{w}(0,0),
$$

applying the Euclidean gradient estimate to $\tilde{w}$ yields

$$
|\nabla \log w(1,0)|=|\nabla \log \tilde{w}(0,0)| \leq C / N_{g} .
$$

This gives the sublinear gradient estimate for $w$ if $N_{g}$ is sufficiently large. The bound on $\mathrm{Hess}_{u}$ follows similarly.

Proposition 11.1 allows us to assume (after rotating so $\nabla u(1,0)=0$ ) that

$$
|\nabla u|+\rho\left|\operatorname{Hess}_{u}\right|+4 \rho|\nabla w| /|w|+\rho^{2}\left|\operatorname{Hess}_{w}\right| /|w| \leq \epsilon<1 /(2 \pi) .
$$

The bound on $\left|\operatorname{Hess}_{w}\right|$ follows from the other bounds and standard elliptic theory.

11.2. Curvature decay. In corollary 1.14 of [CM8], we proved faster than quadratic curvature decay for double-valued minimal graphs whose separation grows sublinearly:

Proposition 11.3. There exists $C$ so if u satisfies the minimal surface equation and (11.12) on

$$
\{1 \leq \rho \leq R,-\pi \leq \theta \leq 3 \pi\},
$$

then on $\left\{1 \leq \rho \leq R^{1 / 2}, 0 \leq \theta \leq 2 \pi\right\}$ we have

$$
\rho\left|H_{e s s_{u}}\right| \leq C \epsilon \rho^{-5 / 12},
$$

and therefore, after possibly rotating $\mathbf{R}^{3}$ (and replacing $u$ ), we get

$$
|\nabla u| \leq C \epsilon \rho^{-5 / 12} .
$$

Of course, Proposition 11.3 is a generalization of Lemma 10.4 which proved curvature decay for annuli. As in the annuli case, the proof uses the quasi-conformality of the Gauss map to deduce a differential inequality. However, the "slit" (i.e., where the double-valued graph does not close up) contributes new terms which are controlled using the estimate for the separation. Notice that the second conclusion (11.15) of the proposition proves a generalization of Bers theorem: Embedded multi-valued minimal graphs have an asymptotic tangent plane.

\section{Sharp estimates on the separation for multi-valued graphs}

We will describe in this section two sharp estimates on the separation of a multivalued minimal graph; see Propositions 12.1 and 12.2 below. These estimates will, unlike the earlier estimates (11.6) and (11.14), require a rapidly growing number of sheets (growing in $\rho$ ).

Suppose for a moment that we are looking at an embedded surface which is the $\infty$-valued graph of a harmonic function so that in particular the separation $w$ is 
a harmonic function which after reflection we may assume is positive. By making the conformal change of coordinates $(\rho, \theta) \rightarrow(\log \rho, \theta)$ we get a positive harmonic

$$
\tilde{w}(x, y)=w\left(\mathrm{e}^{x}, y\right)
$$

defined on the half-plane $\{x \geq 0, y \in \mathbf{R}\}$. By the mean value inequality and the Harnack inequality (since $\tilde{w}$ is positive)

$$
\tilde{w}(y, 0)=\frac{1}{2 \pi y} \int_{\partial D_{y}(y, 0)} \tilde{w} \geq \frac{1}{2 \pi y} \int_{\partial D_{y}(y, 0) \cap D_{1}} \tilde{w} \geq \frac{C}{2 \pi(y+1)} \tilde{w}(0,0) .
$$

Similarly, by an inversion formula one may show that

$$
\tilde{w}(y, 0) \leq \frac{C}{2 \pi(y+1)} \tilde{w}(0,0) .
$$

For the original function $w,(12.2)$ combined with (12.3) gives for some constant $C$ independent of $w$

$$
\frac{1}{C \log \rho} \leq \frac{w(\rho, 0)}{w(1,0)} \leq C \log \rho .
$$

In the case of embedded multi-valued minimal graphs we get similarly:

Proposition 12.1. [CM8]. Let $\Sigma$ be an embedded multi-valued minimal graph of a function $u$ and with a rapidly growing number of sheets, then for the separation $w$ we have for some constant $C$

$$
\frac{1}{C \log \rho} \leq \frac{w(\rho, 0)}{w(1,0)} \leq C \log \rho .
$$

Suppose again for a moment that $u$ and hence $w$ is harmonic. Similarly to (12.2) we get that

$$
\tilde{w}(0, y) \leq C 2 \pi(y+1) \tilde{w}(y, y) .
$$

By the Harnack inequality, we have

$$
\tilde{w}(y, y) \leq C \tilde{w}(y, 0) .
$$

Combining this with (12.6) and (12.3), we get

$$
\tilde{w}(0, y) \leq C\left(y^{2}+1\right) \tilde{w}(0,0) .
$$

For the original function $w$, this gives

$$
\frac{1}{C\left(\theta^{2}+1\right)} \leq \frac{w(\rho, \theta)}{w(\rho, 0)} \leq C\left(\theta^{2}+1\right) .
$$

Again in the case of embedded multi-valued minimal graphs we get similarly:

Proposition 12.2. Let $\Sigma$ be an embedded multi-valued minimal graph of a function $u$ and with a rapidly growing number of sheets, then for the separation $w$ we have for some constant $C$

$$
\frac{1}{C\left(\theta^{2}+1\right)} \leq \frac{w(\rho, \theta)}{w(\rho, 0)} \leq C\left(\theta^{2}+1\right) .
$$


The lower bound in (12.6) for the decay of the separation is sharp. It is achieved for the $\infty$-valued graph of the harmonic function (graphs of multi-valued harmonic functions are good models for multi-valued minimal graphs)

$$
u(\rho, \theta)=\arctan \frac{\theta}{\log \rho} .
$$

Note that the graph of $u$ is embedded and lies in a slab in $\mathbf{R}^{3}$, i.e., $|u| \leq \pi / 2$, and hence in particular is not proper. On the top it spirals into the plane $\left\{x_{3}=\pi / 2\right\}$ and on the bottom into $\left\{x_{3}=-\pi / 2\right\}$, yet it never reaches either of these planes.

QUESTION 1. It would be interesting to construct an infinite-valued exterior solution of the minimal graph equation with the same properties as $\arctan \frac{\theta}{\log \rho}$; i.e., one which spirals infinitely in a slab (see [CM18] for a local example).

\section{Double--valued minimal graphs}

We will now describe how to bound the oscillation of the Gauss map of a double-valued minimal graph. This bound was proven in [CM10]. Rather than give the precise statement here, we will instead illustrate a few of the key ideas by considering the analogous situation for a holomorphic function $f$. Here we are of course thinking of $f$ as being the stereographic projection of the Gauss map. The sublinear growth of the separation, i.e., (11.12) and its integrated form, correspond to

$$
\begin{gathered}
|f|+\rho|\nabla f| \leq \epsilon<1 /(2 \pi), \\
|f(\rho, 2 \pi)-f(\rho, 0)| \leq 2 \pi \epsilon\left(\frac{\delta}{\rho}\right)^{1-\epsilon} .
\end{gathered}
$$

The bound on the oscillation of $f$ (Lemma 13.1 below) now follows by modifying the argument for annuli (Lemma 10.3). Since $f$ does not match up at $\theta=0$ and $2 \pi$, we get an additional term which is estimated using the sublinear growth (13.2).

Lemma 13.1. If $f:\{\delta \leq \rho \leq R, 0 \leq \theta \leq 2 \pi\} \rightarrow \mathbf{C}$ is holomorphic and satisfies (13.1) and (13.2), then

$$
\min _{c \in \mathbf{C}} \max |f-c| \leq \frac{\epsilon}{1-\epsilon}+2 \pi \epsilon .
$$

Proof. (Following Lemma 10.3.) For $\delta \leq s \leq R$, define the circular average

$$
I(s)=(2 \pi)^{-1} \int_{0}^{2 \pi} f(s, \theta) d \theta .
$$

Note that integrating (13.1) gives

$$
|I(s)-f(s, \theta)| \leq 2 \pi \epsilon .
$$

Differentiating (13.4) and using $\frac{\partial f}{\partial \rho}=-i \rho^{-1} \frac{\partial f}{\partial \theta}$ since $f$ is holomorphic, we have

$$
\begin{aligned}
2 \pi s I^{\prime}(s) & =s \int_{0}^{2 \pi} \frac{\partial f(s, \theta)}{\partial \rho} d \theta=-i \int_{0}^{2 \pi} \frac{\partial f(s, \theta)}{\partial \theta} d \theta \\
& =-i[f(s, 2 \pi)-f(s, 0)] .
\end{aligned}
$$

Using the bound (13.2) along the slit, (13.6) gives

$$
s\left|I^{\prime}(s)\right| \leq \epsilon \delta^{1-\epsilon} s^{\epsilon-1} .
$$


In particular, integrating this gives for $\delta \leq \rho \leq R$ that

$$
|I(\rho)-I(\delta)| \leq \epsilon \delta^{1-\epsilon} \int_{\delta}^{\rho} s^{\epsilon-2} d s \leq \epsilon /(1-\epsilon) .
$$

The bound (13.3) follows from (13.5) and (13.8).

Modifying this argument to apply to double-valued minimal graphs introduces new difficulties. In that case, one works directly on the graph (where the Gauss map is holomorphic) and uses averages over "geodesic sectors" rather than circles in the plane. One difficulty is a new term in the analog of (13.6) which results from differentiating the measures of the level sets.

\section{Approximation by standard pieces}

In this section we show that any embedded multi-valued minimal graph has a sub-graph which is close to the sum of a piece of a catenoid and a piece of a helicoid. This generalizes a similar representation for minimal graphs over an annulus given in proposition 1.5 in [CM9]; of course, there was no helicoid term in that case. These results are new and have not appeared in the literature elsewhere.

Recall that half of a catenoid, i.e.,

$$
\left\{\left(x_{1} / s\right)^{2}+\left(x_{2} / s\right)^{2}=\cosh ^{2}\left(x_{3} / s\right), \pm x_{3}>0\right\},
$$

is a minimal graph of

$$
u(z)= \pm s \cosh ^{-1}(|z| / s)
$$

over $\mathbf{C} \backslash D_{s}$. Note that $s \cosh ^{-1}(|z| / s)$ is asymptotic to $s \log [2|z| / s]$. Recall also that half of the helicoid is the multi-valued graph of the function $u$ given in polar coordinates by $u(\rho, \theta)=\theta$. Our approximation result (see Corollary 14.3 below) is therefore that any embedded multi-valued minimal graph has a sub-graph which is close to the graph of a multi-valued function $v$ given by $(a, b, c \in \mathbf{R}$ are constants)

$$
v(\rho, \theta)=a+b \log (\rho / r)+c \theta /(2 \pi) .
$$

As in the two previous sections, $u$ will be a multi-valued function and $w$ will be its separation. For convenience, we will write $S_{r_{1}, r_{2}}^{\theta_{1}, \theta_{2}}$ to denote the "rectangle"

$$
\left\{(\rho, \theta) \mid r_{1} \leq \rho \leq r_{2}, \theta_{1} \leq \theta \leq \theta_{2}\right\} .
$$

We begin with a representation formula for the gradient of an "almost harmonic" function. Recall that if $\Delta u=0$ on an annulus, then the function $f=$ $u_{x}-i u_{y}$ is holomorphic. In particular, $f$ has a Laurent expansion which can be recovered using the Cauchy integral formula. The next lemma uses a variation on this for multi-valued "almost harmonic" functions:

LEMMA 14.1. Given a function $u$ on $S_{1, \sqrt{R}}^{-\pi, 3 \pi}$, set $f=u_{x}-i u_{y}$. If

$$
\begin{aligned}
|f(\zeta)| & \leq C|\zeta|^{-5 / 12}, \\
|\Delta u(\zeta)| & \leq C|\zeta|^{-9 / 4}, \\
\rho|\nabla w| /|w| & \leq \epsilon<1 /(2 \pi),
\end{aligned}
$$

then for $r_{1} \geq 1$ and $\zeta \in S_{2 r_{1}, \sqrt{R} / 2}^{0,2 \pi}$

$$
f(\zeta)=(b+i c /(2 \pi)) \zeta^{-1}+g(\zeta)
$$


where $b, c \in \mathbf{R}$ and

$$
|g(\zeta)| \leq C_{1} R^{-5 / 24}+C_{1} r_{1}^{-1 / 4}|\zeta|^{-1}+C_{1} \epsilon r_{1}^{-1}\left|w\left(r_{1}, 0\right)\right| .
$$

Proof. We will first use the Cauchy integral formula (this is just Stokes' theorem applied to the one-form $f(z) /(z-\zeta) d z)$ on the domain $S_{1, \sqrt{R}}^{0,2 \pi}$ to get a representation formula on $S_{2 r_{1}, \sqrt{R} / 2}^{\pi / 2,3 \pi / 2}$. Assume that $\zeta \in S_{2 r_{1}, \sqrt{R} / 2}^{\pi / 2,3 \pi / 2}$. The Cauchy integral formula gives

$$
\begin{aligned}
& 2 \pi i f(\zeta)=\int_{S_{\sqrt{R}, \sqrt{R}}^{0,2 \pi}} \frac{f(z)}{z-\zeta} d z-\int_{S_{1,1}^{0,2 \pi}} \frac{f(z)}{z-\zeta} d z-i \int_{S_{1, \sqrt{R}}^{0,2 \pi}} \frac{\Delta u}{z-\zeta} \\
& +\left(\int_{\sqrt{R}}^{1} \frac{f(\rho, 2 \pi)}{\rho-\zeta} d \rho+\int_{1}^{\sqrt{R}} \frac{f(\rho, 0)}{\rho-\zeta} d \rho\right) .
\end{aligned}
$$

The first two terms correspond to the usual formula for annuli, the third term vanishes when $f$ is holomorphic, and the last term arises since $f$ is multi-valued. (The first three are almost identical to the corresponding ones in lemma 1.7 in [CM9].) To prove the lemma, we will show that the first term is small and the other three are small after subtracting a multiple of $1 / \zeta$.

First, $|f(z)| \leq C|z|^{-5 / 12}$ and $|\zeta| \leq \sqrt{R} / 2$ give that

$$
\left|\int_{S_{\sqrt{R}, \sqrt{R}}^{0,2 \pi}} \frac{f(z)}{z-\zeta} d z\right| \leq 4 \pi \sup _{\substack{S_{\sqrt{R}, \sqrt{R}}^{0,2 \pi}\\}}|f| \leq 4 \pi C R^{-5 / 24} .
$$

For the remaining terms, we will use the identity

$$
\frac{1}{z-\zeta}=\frac{-1}{\zeta}+\frac{z}{\zeta(z-\zeta)} \text {. }
$$

Second, using (14.12), $2 \leq|\zeta|$, and $|f| \leq C$,

$$
\left|\int_{S_{1,1}^{0,2 \pi}} \frac{f(z)}{z-\zeta} d z+\frac{1}{\zeta} \int_{S_{1,1}^{0,2 \pi}} f(z) d z\right| \leq \frac{2}{|\zeta|^{2}} \int_{S_{1,1}^{0,2 \pi}}|z f(z)| \leq \frac{4 \pi C}{|\zeta|^{2}} .
$$

To bound the third integral, we separate out the disk of radius $|\zeta| / 2$ about $\zeta$ and divide the remainder into two regions using the circle of radius $r_{1}$. Using (14.12), $2 r_{1} \leq|\zeta|$, and $|\Delta u(z)| \leq C|z|^{-9 / 4}$, we get

$$
\begin{aligned}
& \left|\int_{S_{1, \sqrt{R}}^{0,2 \pi}} \frac{\Delta u}{z-\zeta}+\frac{1}{\zeta} \int_{S_{1, r_{1}}^{0,2 \pi}} \Delta u\right| \leq 4 \pi C|\zeta|^{-2} \int_{1}^{r_{1}} r^{-1 / 4} d r+2^{9 / 4} C \int_{D_{\frac{|\zeta|}{2}}(\zeta)} \frac{|\zeta|^{-9 / 4}}{|z-\zeta|} \\
& +4 \pi C|\zeta|^{-1} \int_{r_{1}}^{\sqrt{R}} r^{-5 / 4} d r \\
& \leq 12 \pi C|\zeta|^{-5 / 4}+16 \pi C r_{1}^{-1 / 4}|\zeta|^{-1} \text {. }
\end{aligned}
$$

Finally, this leaves the slit (i.e., the terms which arise because $u$ is not welldefined over the annulus), where we divide the integral into three parts

$$
\begin{aligned}
\int_{1}^{\sqrt{R}} & \frac{f(\rho, 2 \pi)-f(\rho, 0)}{\rho-\zeta} d \rho=\int_{r_{1}}^{\sqrt{R}} \frac{f(\rho, 2 \pi)-f(\rho, 0)}{\rho-\zeta} d \rho \\
& -\zeta^{-1} \int_{1}^{r_{1}}(f(\rho, 2 \pi)-f(\rho, 0)) d \rho+\int_{1}^{r_{1}} \frac{\rho(f(\rho, 2 \pi)-f(\rho, 0))}{\zeta(\rho-\zeta)} d \rho
\end{aligned}
$$


Using $\rho|\nabla w| /|w| \leq \epsilon$, we get for $\rho \geq r_{1}$ that

$$
|f(\rho, 2 \pi)-f(\rho, 0)|=|\nabla w(\rho, 0)| \leq \epsilon \frac{|w|(\rho, 0)}{\rho} \leq \epsilon\left(\frac{\rho}{r_{1}}\right)^{\epsilon} \frac{|w|\left(r_{1}, 0\right)}{\rho} .
$$

Note that the real part of $\zeta$ is negative since $\zeta \in S_{2 r_{1}, \sqrt{R} / 2}^{\pi / 2,3 \pi / 2}$ and hence $|\rho-\zeta| \geq \rho$.

To bound the first term in (14.15), we use this and (14.16) to get

$$
\left|\int_{r_{1}}^{\sqrt{R}} \frac{f(\rho, 2 \pi)-f(\rho, 0)}{\rho-\zeta} d \rho\right| \leq \frac{\epsilon}{r_{1}^{\epsilon}}|w|\left(r_{1}, 0\right) \int_{r_{1}}^{\sqrt{R}} \rho^{\epsilon-2} d \rho \leq 2 \epsilon \frac{|w|\left(r_{1}, 0\right)}{r_{1}}
$$

Similarly, to bound the third, use $\rho|\nabla w| /|w| \leq \epsilon$ to get

$$
\begin{aligned}
\left|\int_{1}^{r_{1}} \frac{\rho(f(\rho, 2 \pi)-f(\rho, 0))}{\zeta(\rho-\zeta)} d \rho\right| & \leq \epsilon r_{1}^{-2} \int_{1}^{r_{1}}|w|(\rho, 0) d \rho \\
& \leq \epsilon r_{1}^{-2} \int_{1}^{r_{1}}|w|\left(r_{1}, 0\right)\left(r_{1} / \rho\right)^{\epsilon} d \rho \leq 2 \epsilon \frac{|w|\left(r_{1}, 0\right)}{r_{1}}
\end{aligned}
$$

Putting all of this together, we now get the desired representation formula (14.8) and remainder estimate (14.9) for $\zeta \in S_{2 r_{1}, \sqrt{R} / 2}^{\pi / 2,3 \pi / 2}$. Namely, we set $g=g_{1}+g_{2}+g_{3}$ and

$$
2 \pi i\left(b+i \frac{c}{2 \pi}\right)=\int_{S_{1,1}^{0,2 \pi}} f(z) d z+\int_{1}^{r_{1}}(f(\rho, 2 \pi)-f(\rho, 0)) d \rho+i \int_{S_{1, r_{1}}^{0,2 \pi}} \Delta u,
$$

$$
\begin{aligned}
& 2 \pi i g_{1}(\zeta)=\int_{S_{\sqrt{R}, \sqrt{R}}^{0,2 \pi}} \frac{f(z)}{z-\zeta} d z \\
& 2 \pi i g_{2}(\zeta)=-\int_{r_{1}}^{\sqrt{R}} \frac{f(\rho, 2 \pi)-f(\rho, 0)}{\rho-\zeta} d \rho-\int_{1}^{r_{1}} \frac{f(\rho, 2 \pi)-f(\rho, 0)}{\zeta(\rho-\zeta)} \rho d \rho, \\
& 2 \pi i g_{3}(\zeta)=-\int_{S_{1,1}^{0,2 \pi}} \frac{f(z)}{z-\zeta} d z-\frac{1}{\zeta} \int_{S_{1,1}^{0,2 \pi}} f(z) d z-i \int_{S_{1, \sqrt{R}}^{0,2 \pi}} \frac{\Delta u}{z-\zeta}-\frac{i}{\zeta} \int_{S_{1, r_{1}}^{0,2 \pi}} \Delta u .
\end{aligned}
$$

We can repeat this by integrating over $S_{1, \sqrt{R}}^{-\pi / 2,3 \pi / 2}$ and $S_{1, \sqrt{R}}^{\pi / 2,5 \pi / 2}$ to get similar representations on $S_{2 r_{1}, \sqrt{R} / 2}^{0, \pi}$ and $S_{2 r_{1}, \sqrt{R} / 2}^{\pi, 2 \pi}$ (with different values of $b$ and $c$ ). By continuity, the same representation holds on all three regions (since they overlap), giving the lemma.

Definition 14.2. Fix $\mu>0$. A standard piece $\Sigma_{v}$ on the scale $r$ is a graph of $v$ over $S_{r, \mu r}^{-\pi, 3 \pi}(y)$ given in polar coordinates $\left(\rho_{p}, \theta_{p}\right)$ centered at $p$ by

$$
v\left(\rho_{p}, \theta_{p}\right)=a+b \log \left(\rho_{p} / r\right)+c \theta_{p} /(2 \pi) .
$$

The constant $a$ is the "plane coefficient", $b$ is the "catenoid coefficient" and $c$ is the "helicoid coefficient" (this gives the separation $w$ ). Note that $v$ is harmonic.

COROLlaRY 14.3. Let $u$ be a solution of the minimal graph equation on $S_{1 / 2, R}^{-3 \pi, 5 \pi}$ with $w<0$ satisfying (11.12) and $|u| \leq \epsilon \rho$. There is a rotation of $\mathbf{R}^{3}$ so given $r_{1}>2$, we get a standard piece

$$
v=a+b \log \rho+c \theta /(2 \pi),
$$


for $a, b, c \in \mathbf{R}$ with

$$
\sup _{S_{r_{1}, \mu r_{1}}^{0,22 \pi}}|u(\rho, \theta)-v(\rho, \theta)| \leq C_{2} \mu \epsilon|w|\left(r_{1}, 0\right)+C_{2} \mu r_{1} R^{-5 / 24}+C_{2} \mu r_{1}^{-1 / 4} .
$$

Proof. Corollary 1.14 in [CM8] give a rotation of $\mathbf{R}^{3}$ so that for $z \in S_{1, \sqrt{R}}^{-2 \pi, 4 \pi}$

$$
|\nabla u(z)|+|z|\left|\operatorname{Hess}_{u}(z)\right| \leq C \epsilon|z|^{-5 / 12} .
$$

Since, by (1.6) of $[\mathbf{C M 8}],|\Delta u| \leq|\nabla u|^{2}\left|\operatorname{Hess}_{u}\right|$, (14.23) gives on $S_{1, \sqrt{R}}^{-2 \pi, 4 \pi}$ that

$$
|\Delta u(z)| \leq C \epsilon^{3}|z|^{-9 / 4}
$$

Note that

$$
\begin{aligned}
(\log \rho)_{x}-i(\log \rho)_{y} & =1 /(x+i y), \\
\theta_{x}-i \theta_{y} & =i /(x+i y) .
\end{aligned}
$$

Using this and Lemma 14.1, we get that on $S_{r_{1}, \mu r_{1}}^{0,2 \pi}$

$$
|\nabla(u-b \log \rho-c \theta /(2 \pi))| \leq C_{1}\left(R^{-5 / 24}+2 r_{1}^{-1 / 4} \rho^{-1}+2 \epsilon \frac{\left|w\left(r_{1}, 0\right)\right|}{r_{1}}\right)
$$

Integrating (14.27) gives (14.22).

QUESTION 2. Corollary 14.3 shows that embedded multi-valued minimal graphs are closely approximated by helicoids (plus a catenoid term) on each scale. However, a priori, the helicoid coefficient can change from scale to scale (the point of [CM10] is that the axis of the helicoid does not change so that all are vertical). It would be interesting to estimate how quickly this helicoid coefficient can change and construct examples demonstrating this.

\section{Part 3. Regularity theory}

In this part, we survey some of the key ideas in classical regularity theory, recent developments on embedded minimal disks, and some global results for minimal surfaces in $\mathbf{R}^{3}$. Sections 15 and 16 focus on Reifenberg type conditions, where a set is assumed to be close to a plane at all points and at all scales ("close" is in the Hausdorff or Gromov-Hausdorff sense and is defined in Section 15). This condition automatically gives Hölder regularity (and hence higher regularity if the set is also a weak solution to a natural equation). Section 17 surveys the role of monotonicity and scaling in regularity theory, including $\epsilon$-regularity theorems (such as Allard's theorem) and tangent cone analysis (such as Almgren's refinement of Federer's dimension reducing). Section 18 briefly reviews recent results of the authors for embedded minimal disks, developing a regularity theory in a setting where the classical methods cannot be applied and in particular where there is no monotonicity. The estimates and ideas discussed in Section 18 have applications to the global theory of minimal surfaces in $\mathbf{R}^{3}$. In Section 19, we give a quick tour of some recent results in this classical, but rapidly developing, area. 


\section{Hausdorff and Gromov-Hausdorff distances}

Recall that the Hausdorff distance between two subsets $A$ and $B$ of a Euclidean space is no greater than $\delta>0$ provided that each is contained in a $\delta$-neighborhood of the other.

There is a natural generalization of this classical Hausdorff distance between subsets of Euclidean space to a distance function on all metric spaces. This is the Gromov-Hausdorff distance and it gives a good tool to study metric spaces.

Suppose that $\left(X, d_{X}\right)$ and $\left(Y, d_{Y}\right)$ are two compact metric spaces. We say that the Gromov-Hausdorff distance between them is at most $\epsilon>0$ if there exist maps $f: X \rightarrow Y$ and $g: Y \rightarrow X$ such that

$$
\begin{gathered}
\forall x_{1}, x_{2} \in X:\left|d_{X}\left(x_{1}, x_{2}\right)-d_{Y}\left(f\left(x_{1}\right), f\left(x_{2}\right)\right)\right|<\epsilon, \\
\forall x \in X: d_{X}(x, g \circ f(x))<\epsilon,
\end{gathered}
$$

and the two symmetric properties in $Y$ hold. The Gromov-Hausdorff distance between $X$ and $Y$, denoted by $d_{G H}(X, Y)$, is then the infimum of all such $\epsilon$.

Using this topology we can then say that a sequence of metric space converges to another metric space. For noncompact metric spaces there is a more useful notion of convergence which is essentially convergence on compact subsets. Namely, if $\left(X_{i}, x_{i}, d_{X_{i}}\right)$ is a pointed sequence of metric spaces then we say that $\left(X_{i}, x_{i}, d_{X_{i}}\right)$ converges to some pointed metric space $\left(X, x, d_{X}\right)$ in the pointed Gromov-Hausdorff topology if for all $r_{0}>0$ fixed the compact metric spaces $B_{r_{0}}\left(x_{i}\right)$ converges to $B_{r_{0}}(x)$.

Gromov's compactness theorem is the statement that any pointed sequence, $\left(M_{i}^{n}, m_{i}\right)$, of $n$-dimensional manifolds, with

$$
\operatorname{Ric}_{M_{i}^{n}} \geq(n-1) \Lambda
$$

has a subsequence, $\left(M_{j}^{n}, m_{j}\right)$, which converges in the pointed Gromov-Hausdorff topology to some length space $\left(M_{\infty}, m_{\infty}\right)$.

The proof of this compactness theorem relies only on the volume comparison theorem. In fact it only uses the volume doubling which is implied by the volume comparison.

\section{Reifenberg type conditions}

One concept or idea that often plays a central role in regularity theory is that of being Reifenberg flat, a notion introduced by E. R. Reifenberg [Re]. In the case of Reifenberg it was introduced to measure the closeness of a subset of Euclidean space to being an affine space. The deviation was measured on all scales and a number was assigned which was the maximal scale invariant Hausdorff distance to a affine plane, see $[\mathbf{T o}],[\mathbf{S e}],[\mathbf{D a T o}],[\mathbf{D a K e T o}]$ for interesting results in this classical direction.

To explain this point about the importance of this condition further we recall the following definition from $[\mathbf{C h C 1}$ ] which was inspired by the classical work of Reifenberg.

Let $\left(Z, d_{Z}\right)$ be a complete metric space. We will say that $Z$ satisfies the $(\epsilon, \rho, n)$ $\mathcal{G}_{\mathcal{R}}$ (or Generalized Reifenberg) condition at $z \in Z$ if for all $0<\sigma<\rho$ and all $y \in B_{\rho-\sigma}(z)$,

$$
d_{G H}\left(B_{\sigma}(y), B_{\sigma}(0)\right)<\epsilon \sigma,
$$


where $B_{r_{0}}(0) \subset \mathbf{R}^{n}$.

Theorem 16.1. (Appendix of [ChC2].) Given $\epsilon>0$ and $n \geq 2$, there exists $\delta>0$ such that if $\left(Z, d_{Z}\right)$ is a complete metric space and $Z$ satisfies the $\left(\delta, r_{0}, n\right)$ $\mathcal{G}_{\mathcal{R}}$ condition at $z \in Z$ then there exists a bi-Hölder homeomorphism $\Phi: B_{\frac{r_{0}}{2}}(z) \rightarrow$ $B_{\frac{r_{0}}{2}}(0)$ such that for all $z_{1}, z_{2} \in Z$,

$$
r_{0}^{-\epsilon}\left|\Phi\left(z_{1}\right)-\Phi\left(z_{2}\right)\right|^{1+\epsilon} \leq d_{Z}\left(z_{1}, z_{2}\right) \leq r_{0}^{\epsilon}\left|\Phi\left(z_{1}\right)-\Phi\left(z_{2}\right)\right|^{1-\epsilon} .
$$

Here is one example where such a Reifenberg type condition naturally come up; see $[\mathbf{C 2}],[\mathbf{C h C 1}]$ for more on this.

Theorem 16.2. ([C1], $[\mathbf{C h C 1}])$. Given $\epsilon>0$ and $n \geq 2$, there exist $\delta=$ $\delta(\epsilon, n)>0$ and $\rho=\rho(\epsilon, n)>0$, such that if $M^{n}$ has $\operatorname{Ric}_{M^{n}} \geq-(n-1)$ and $0<r_{0} \leq \rho$ with

$$
d_{G H}\left(B_{2 r_{0}}(x), B_{2 r_{0}}(0)\right)<\delta r_{0},
$$

or

$$
\operatorname{Vol}\left(B_{2 r_{0}}(x)\right) \geq(1-\delta) V_{0}^{n}\left(2 r_{0}\right),
$$

then $M^{n}$ satisfies the $\left(\epsilon, r_{0}, n\right)-\mathcal{G}_{\mathcal{R}}$ condition at $x$.

\section{Monotonicity and regularity theory}

As we have already seen, the monotonicity of a "scale-invariant energy" has played a key role in the regularity theory for geometric variational problems. In many cases, this monotonicity is useful for establishing two key tools:

1. An $\epsilon$-regularity theorem which guarantees that a weak solution is actually smooth when the scale-invariant energy is small.

2. The existence of tangent cones which are dilation invariant.

In this section, we briefly review the role of monotonicity in regularity theory, emphasizing these two tools, and give some examples.

To keep things concrete, it may be useful to mention some examples of variational problems and their scale-invariant energies, some of which we have already encountered. The primary example is for a minimal $k$-dimensional submanifold; in this case, the appropriate scale-invariant energy is the density

$$
\Theta_{x_{0}}(s)=\frac{\operatorname{Vol}\left(B_{s}\left(x_{0}\right) \cap \Sigma\right)}{\operatorname{Vol}\left(B_{s} \subset \mathbf{R}^{k}\right)} .
$$

There are many other examples, including

- If $\Sigma \subset \mathbf{R}^{n}$ is a (2-dimensional) minimal surface, then another possible scaleinvariant energy is $\int_{B_{r}}|A|^{2}$.

- If $u: \mathbf{R}^{k} \rightarrow M$ is a harmonic map, then the scale-invariant energy is $r^{2-k} \int_{B_{r}}|\nabla u|^{2}$, see $[\mathbf{S c U} \mathbf{U h}]$.

- If $M^{n}$ has non-negative Ricci curvature, then the scale-invariant energy is the volume density $r^{-n} \operatorname{Vol}\left(B_{r}\left(x_{0}\right) \subset M\right)$ - here, the monotonicity goes the opposite direction.

- If $A$ is a Yang-Mills connection on $M^{k}$ with curvature $F_{A}$, then the scaleinvariant energy is $r^{4-k} \int_{B_{r}}\left|F_{A}\right|^{2}$, see $[\mathbf{P r}]$. 
Interestingly, there are also parabolic analogs for all of these examples (cf. Huisken's monotonicity formula for the mean curvature flow, i.e., Theorem 6.2 or M. Struwe's monotonicity for the harmonic map heat flow, theorem 1.10 in $[\mathbf{S t}])$.

Using this monotonicity, we can define the density $\Theta_{x_{0}}$ at the point $x_{0}$ to be the limit as $r \rightarrow 0$ of the scale-invariant energy in $B_{r}\left(x_{0}\right)$. It follows easily from the monotonicity that the density is semi-continuous.

Remark 17.1. These energies are all scale-invariant in the sense that if a solution is rescaled by a factor $\lambda$, then the energy of the new solution on a ball of radius $\lambda r$ is the same as the original solution on a ball of radius $r$. It is not difficult to find scale-invariant quantities - the difficulty lies in finding one that is monotone and can be estimated. For example, if $u: \mathbf{R}^{k} \rightarrow M$ is a harmonic map, then $\int|\nabla u|^{k}$ is scale-invariant and monotone; however, it is not at all clear that there should be an a priori bound on this when $k>2$.

17.1. $\epsilon-$-regularity and the singular set. This monotonicity is then important in proving an $\epsilon$-regularity theorem. Recall that an $\epsilon$-regularity theorem gives that a weak (or generalized) solution is actually smooth at a point if the scale-invariant energy is small enough there. The standard example is the Allard regularity theorem:

TheOREM 17.2. [Al] There exists $\delta(k, n)>0$ such that if $\Sigma \subset \mathbf{R}^{n}$ is a $k$ rectifiable stationary varifold (with density at least one a.e.), $x_{0} \in \Sigma$, and

$$
\Theta_{x_{0}}=\lim _{r \rightarrow 0} \frac{\operatorname{Vol}\left(B_{r}\left(x_{0}\right) \cap \Sigma\right)}{\operatorname{Vol}\left(B_{r} \subset \mathbf{R}^{k}\right)}<1+\delta,
$$

then $\Sigma$ is smooth in a neighborhood of $x_{0}$.

Another well-known example is the $\epsilon$-regularity theorem of Schoen and K. Uhlenbeck for harmonic maps:

TheOREM 17.3. [ScUh] There exists $\epsilon(k, N)>0$ such that if $u: B_{r} \subset \mathbf{R}^{k} \rightarrow N$ is an energy minimizing map and

$$
r^{2-k} \int_{B_{r}}|\nabla u|^{2}<\epsilon
$$

then $u$ is smooth in a neighborhood of 0 and $|\nabla u|(0) \leq C r^{-1}$.

See $[\mathbf{C h C T i}$ ] for $\epsilon$-regularity results for limits of Kähler-Einstein metrics and their applications to regularity of such limit spaces and see F.H. Lin, $[\mathbf{L n}]$, for recent developments on the regularity of harmonic maps which are not minimizing.

Remark 17.4. The $\epsilon$-regularity theorem is closely related to a priori estimates when the energy is small (cf. Section 9). In one direction, combining the regularity with a compactness argument usually directly gives an a priori estimate.

The singular set $\mathcal{S}$ is defined to be the set where the scale-invariant energy is not small. The first application of these $\epsilon-$ regularity theorems is some control on the singular set. For example, the semi-continuity of the density immediately gives that $\mathcal{S}$ is closed. In order to bound the size of the singular set (e.g., the Hausdorff measure), we combine the $\epsilon$-regularity with simple covering arguments:

LEMMA 17.5. If $u: B_{1} \subset \mathbf{R}^{k} \rightarrow M$ is an energy minimizing (harmonic) map, then the $(k-2)$-dimensional Hausdorff measure of $\mathcal{S}$ is zero. 
Proof. Given $\delta>0$ and $x \in \mathcal{S}$, then the $\epsilon$-regularity theorem (Theorem 17.3) yields a ball $B_{r_{x}}(x)$ so that

$$
0<\epsilon \leq r_{x}^{2-k} \int_{B_{r_{x}}(x)}|\nabla u|^{2} .
$$

Using the 5 -times covering lemma, we can find a disjoint collection of balls $B_{r_{i}}\left(x_{i}\right)$ so that

$$
\begin{aligned}
& \epsilon \leq r_{i}^{2-k} \int_{B_{r_{i}}\left(x_{i}\right)}|\nabla u|^{2}, \\
& \mathcal{S} \subset \cup_{i} B_{5 r_{i}}\left(x_{i}\right) .
\end{aligned}
$$

Since the balls $B_{r_{i}}\left(x_{i}\right)$ are disjoint, (17.5) implies that

$$
\epsilon \sum_{i} r_{i}^{k-2} \leq \sum_{i} \int_{B_{r_{i}}\left(x_{i}\right)}|\nabla u|^{2} \leq \int_{\cup_{i} B_{r_{i}}\left(x_{i}\right)}|\nabla u|^{2} .
$$

Combining this with (17.6), we get a uniform bound for the $(k-2)$-dimensional Hausdorff measure of $\mathcal{S}$. In particular, $\mathcal{S}$ has Lebesgue measure zero.

Finally, we show that the $(k-2)$-dimensional Hausdorff measure of $\mathcal{S}$ is zero (and not just finite). First, notice that as $\delta \rightarrow 0$, the Lebesgue measure of $\cup_{i} B_{r_{i}}\left(x_{i}\right)$ goes to zero. Since $|\nabla u|^{2}$ is an $L^{1}$ function, the dominated convergence theorem implies that

$$
\lim _{\delta \rightarrow 0} \int_{\cup_{i} B_{r_{i}}\left(x_{i}\right)}|\nabla u|^{2}=0 .
$$

Substituting this back into (17.7), gives the claim.

This preliminary analysis of the singular set can be refined by doing a so-called tangent cone analysis.

17.2. Tangent cone analysis. Each of these variational problems comes with a natural scaling which preserves the space of solutions. For example, if $\Sigma \subset \mathbf{R}^{n}$ is a minimal submanifold, then so is

$$
\Sigma_{y, \lambda}=\left\{y+\lambda^{-1}(x-y) \mid x \in \Sigma\right\} .
$$

(To see this, simply note that this scaling multiplies the principal curvatures by $\lambda$.) Similarly, if $u: \mathbf{R}^{n} \rightarrow M$ is an energy minimizing map, then so is the map $u_{y, \lambda}$ defined by

$$
u_{y, \lambda}(x)=u(y+\lambda(x-y)) .
$$

Suppose now that we fix the point $y$ and take a sequence $\lambda_{j} \rightarrow 0$. The monotonicity formula (for either area or energy) bounds the density of the rescaled solution, allowing us to extract a convergent subsequence and limit. This limit, which is called a tangent cone at $y$, achieves equality in the monotonicity formula and, hence, must be homogeneous (i.e., invariant under dilations about $y$ ).

The usefulness of tangent cone analysis in regularity theory is based on two key facts. For simplicity, we illustrate these when $\Sigma \subset \mathbf{R}^{n}$ is an area minimizing hypersurface. (See $[\mathbf{C h C 1}]$ for similar results for singular limit spaces of manifolds with lower Ricci curvature bounds.) First, if any tangent cone at $y$ is a hyperplane $\mathbf{R}^{n-1}$, then $\Sigma$ is smooth in a neighborhood of $y$. This follows easily from the Allard regularity theorem since the density at $y$ of the tangent cone is the same as the 
density at $y$ of $\Sigma$. The second key fact, known as "dimension reducing," is due to Almgren, [Am2], and is a refinement of an argument of Federer. To state this, we first stratify the singular set $\mathcal{S}$ of $\Sigma$ into subsets

$$
\mathcal{S}_{0} \subset \mathcal{S}_{1} \subset \cdots \subset \mathcal{S}_{n-2}
$$

where we define $\mathcal{S}_{i}$ to be the set of points $y \in \mathcal{S}$ so that any linear space contained in any tangent cone at $y$ has dimension at most $i$. (Note that $\mathcal{S}_{n-1}=\emptyset$ by the previous fact.) The dimension reducing argument then gives that

$$
\operatorname{dim}\left(\mathcal{S}_{i}\right) \leq i,
$$

where dimension means the Hausdorff dimension. In particular, the solution of the Bernstein problem then gives codimension 7 regularity of $\Sigma$, i.e., $\operatorname{dim}(\mathcal{S}) \leq n-8$. See lecture 2 in $[\mathbf{S i 3}]$ for a proof of (17.12).

Remark 17.6. Using that the $(k-2)$-dimensional Hausdorff measure of $\mathcal{S}$ is zero for an energy minimizing map $u: \mathbf{R}^{k} \rightarrow M$ by Lemma 17.5, it is not hard to see that $\mathcal{S}_{k-2}=\emptyset$. In particular, we get that $\mathcal{S}$ is at most $(k-3)$-dimensional

This approach has been applied fruitfully to many problems since it requires only a natural scaling, a monotonicity formula, and a compactness theorem. A variation of this, giving tangent flows rather than tangent cones, has also been useful in parabolic problems (see, e.g., $[\mathbf{W}]$ for an application to mean curvature flow).

Note that the tangent cones produced in this way may very well depend on the particular convergent subsequence. In some cases, one can prove uniqueness of the tangent cone and this is often quite useful (see, for instance, section 3.4 in [Si3] for one such application). However, in many settings tangent cones are not unique; see, for instance, $[\mathbf{P e} 4],[\mathrm{ChC1}]$.

\section{Embedded minimal disks}

We next survey recent results of the authors for embedded minimal disks. The main result is a compactness and singular convergence theorem (Theorem 18.1 below) in a setting where the classical methods cannot be applied. In particular, there is no useful monotonicity formula or natural a priori bound. The main tools for overcoming these difficulties are a "classification of singularities" which describes a neighborhood of points of large curvature (Theorem 18.2) and our one-sided curvature estimate (Theorem 18.3 below). We will keep things brief here, attempting to highlight a few key points. We refer to [CM22] for a more detailed survey.

As we will see, a fundamental theorem about embedded minimal disks is that such a disk is either a minimal graph or can be approximated by a piece of a rescaled helicoid depending on whether the curvature is small or not; see Theorem 18.1 below. To avoid tedious dependence of various quantities we state this, our main result, not for a single embedded minimal disk with sufficiently large curvature at a given point but instead for a sequence of such disks where the curvatures are blowing up. Theorem 18.1 says that a sequence of embedded minimal disks mimics the following behavior of a sequence of rescaled helicoids:

Consider the sequence $\Sigma_{i}=a_{i} \Sigma$ of rescaled helicoids where $a_{i} \rightarrow 0$. (That is, rescale $\mathbf{R}^{3}$ by $a_{i}$, so points that used to be distance $d$ apart will in the rescaled $\mathbf{R}^{3}$ be distance $a_{i} d$ apart.) The curvatures of 
this sequence of rescaled helicoids are blowing up along the vertical axis. The sequence converges (away from the vertical axis) to a foliation by flat parallel planes. The singular set $\mathcal{S}$ (the axis) then consists of removable singularities.

Let now $\Sigma_{i} \subset B_{2 R}$ be a sequence of embedded minimal disks with $\partial \Sigma_{i} \subset \partial B_{2 R}$. Clearly (after possibly going to a subsequence) either (A) or (B) occur:

(A) $\sup _{B_{R} \cap \Sigma_{i}}|A|^{2} \leq C<\infty$ for some constant $C$.

(B) $\sup _{B_{R} \cap \Sigma_{i}}|A|^{2} \rightarrow \infty$.

In (A) (by a standard argument) the intrinsic ball $\mathcal{B}_{s}\left(y_{i}\right)$ is a graph for all $y_{i} \in$ $B_{R} \cap \Sigma_{i}$, where $s$ depends only on $C$. Thus the main case is (B) which is the subject of the next theorem.

Using the notion of multi-valued graphs, the lamination theorem (the main theorem of $[\mathbf{C M} 6])$, can now be stated:

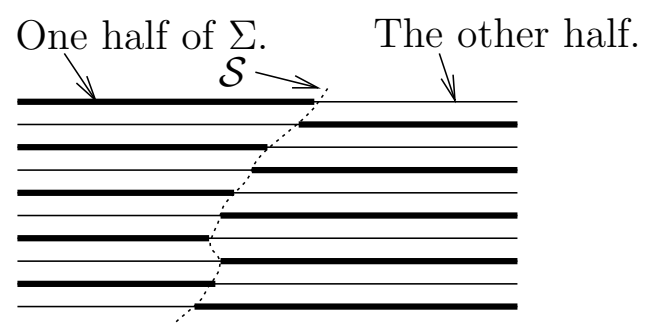

FiguRE 3. Theorem 18.1 - the singular set, $\mathcal{S}$, and the two multi-valued graphs.

Theorem 18.1. (Theorem 0.1 in [CM6]). See fig. 3. Let $\Sigma_{i} \subset B_{R_{i}}=$ $B_{R_{i}}(0) \subset \mathbf{R}^{3}$ be a sequence of embedded minimal disks with $\partial \Sigma_{i} \subset \partial B_{R_{i}}$ where $R_{i} \rightarrow \infty$. If

$$
\sup _{B_{1} \cap \Sigma_{i}}|A|^{2} \rightarrow \infty
$$

then there exists a subsequence, $\Sigma_{j}$, and a Lipschitz curve $\mathcal{S}: \mathbf{R} \rightarrow \mathbf{R}^{3}$ such that after a rotation of $\mathbf{R}^{3}$ :

1. $x_{3}(\mathcal{S}(t))=t$. (That is, $\mathcal{S}$ is a graph over the $x_{3}$-axis.)

2. Each $\Sigma_{j}$ consists of exactly two multi-valued graphs away from $\mathcal{S}$ (which spiral together).

3. For each $1>\alpha>0, \Sigma_{j} \backslash \mathcal{S}$ converges in the $C^{\alpha}$-topology to the foliation, $\mathcal{F}=\left\{x_{3}=t\right\}_{t}$, of $\mathbf{R}^{3}$.

4. $\sup _{B_{r}(\mathcal{S}(t)) \cap \Sigma_{j}}|A|^{2} \rightarrow \infty$ for all $r>0, t \in \mathbf{R}$. (The curvatures blow up along S.)

In (2), (3) that $\Sigma_{j} \backslash \mathcal{S}$ are multi-valued graphs and converges to $\mathcal{F}$ means that for each compact subset $K \subset \mathbf{R}^{3} \backslash \mathcal{S}$ and $j$ sufficiently large $K \cap \Sigma_{j}$ consists of multi-valued graphs over (part of) $\left\{x_{3}=0\right\}$ and $K \cap \Sigma_{j} \rightarrow K \cap \mathcal{F}$ in the sense of graphs. 
Theorem 18.1 (as many of the other results discussed below) is modelled by the helicoid and its rescalings. Take a sequence $\Sigma_{i}=a_{i} \Sigma$ of rescaled helicoids where $a_{i} \rightarrow 0$. The curvatures of this sequence are blowing up along the vertical axis. The sequence converges (away from the vertical axis) to a foliation by flat parallel planes. The singular set $\mathcal{S}$ (the axis) then consists of removable singularities.

We now come to our key results for embedded minimal disks. These are some of the main ingredients in the proof of Theorem 18.1. The first says that if the curvature of such a disk $\Sigma$ is large at some point $x \in \Sigma$, then nearby $x$ a multi-valued graph forms (in $\Sigma$ ) and this extends (in $\Sigma$ ) almost all the way to the boundary. Precisely this is:

Theorem 18.2. (Theorem 0.2 in [CM4]). See fig. 4 and fig. 5. Given $N \in$ $\mathbf{Z}_{+}, \epsilon>0$, there exist $C_{1}, C_{2}>0$ so that the following holds:

Let $0 \in \Sigma^{2} \subset B_{R} \subset \mathbf{R}^{3}$ be an embedded minimal disk with $\partial \Sigma \subset \partial B_{R}$. If for some $R>r_{0}>0$ we have

$$
\max _{B_{r_{0}} \cap \Sigma}|A|^{2} \geq 4 C_{1}^{2} r_{0}^{-2}
$$

then there exists (after a rotation of $\mathbf{R}^{3}$ ) an $N$-valued graph $\Sigma_{g}$ over $D_{R / C_{2}} \backslash D_{2 r_{0}}$ with gradient $\leq \epsilon$ and contained in $\Sigma \cap\left\{x_{3}^{2} \leq \epsilon^{2}\left(x_{1}^{2}+x_{2}^{2}\right)\right\}$.

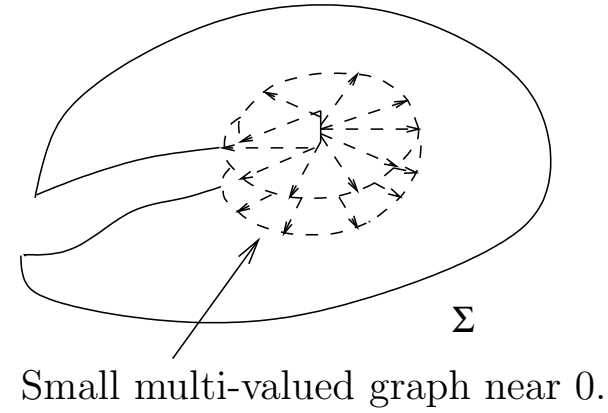

Figure 4. Part 1 of the proof of Theorem 18.2; finding a small multi-valued graph in a disk near a point of large curvature.

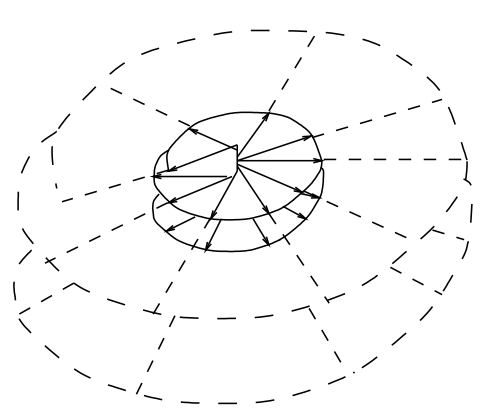

Figure 5. Part 2 of the proof of Theorem 18.2; extending a small multi-valued graph in a disk.

As a consequence of Theorem 18.2, one easily gets that if $|A|^{2}$ is blowing up near 0 for a sequence of embedded minimal disks $\Sigma_{i}$, then there is a sequence of 2-valued graphs $\Sigma_{i, d} \subset \Sigma_{i}$, where the 2-valued graphs start off on a smaller and smaller scale (namely, $r_{0}$ in Theorem 18.2 can be taken to be smaller as the curvature gets larger). Consequently, by the sublinear separation growth, such $2-$ valued graphs collapse and, hence, a subsequence converges to a smooth minimal graph through 0 . To be precise, given any fixed $\rho>0,(11.4)$ bounds the separation $w$ at $(\rho, 0)$ by

$$
|w(\rho, 0)| \leq\left(\frac{\rho}{r_{0}}\right)^{\alpha}\left|w\left(r_{0}, 0\right)\right| \leq 2 \pi \epsilon \rho^{\alpha} r_{0}^{1-\alpha},
$$

and this goes to 0 as $r_{0} \rightarrow 0$ since $\alpha<1$. The bound

$$
\left|w\left(r_{0}, 0\right)\right| \leq 2 \pi \epsilon r_{0}
$$


in (18.2) came from integrating the gradient bound on the graph around the circle of radius $r_{0}$. (Here 0 is a removable singularity for the limit.) Moreover, if the sequence of such disks is as in Theorem 18.1, i.e., if $R_{i} \rightarrow \infty$, then the minimal graph in the limit is entire and hence, by Bernstein's theorem (theorem 1.16 in [CM1]), is a plane.

The second key result is the curvature estimate for embedded minimal disks in a half-space. This theorem says roughly that if an embedded minimal disk lies in a half-space above a plane and comes close to the plane, then it is a graph over the plane. Precisely, this is the following theorem:

Theorem 18.3. (Theorem 0.2 in [CM6]). See fig. 6. There exists $\epsilon>0$, so that if

$$
\Sigma \subset B_{2 r_{0}} \cap\left\{x_{3}>0\right\} \subset \mathbf{R}^{3}
$$

is an embedded minimal disk with $\partial \Sigma \subset \partial B_{2 r_{0}}$, then for all components $\Sigma^{\prime}$ of $B_{r_{0}} \cap \Sigma$ which intersect $B_{\epsilon r_{0}}$ we have

$$
\sup _{\Sigma^{\prime}}\left|A_{\Sigma}\right|^{2} \leq r_{0}^{-2}
$$

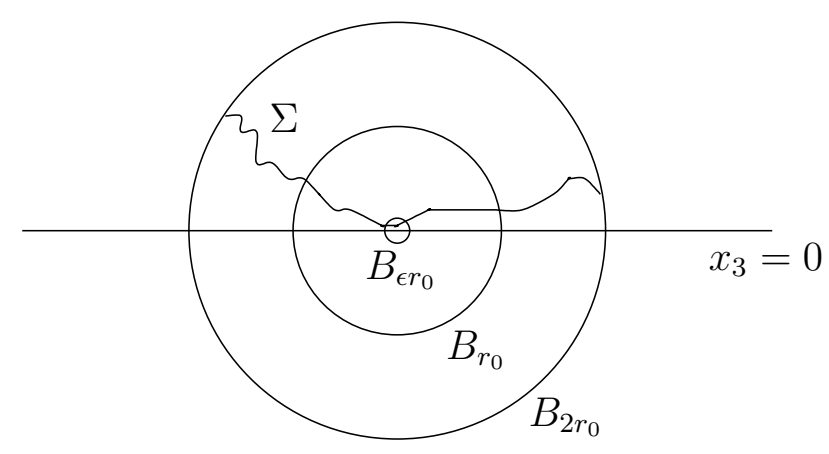

Figure 6. Theorem 18.3 - the onesided curvature estimate for an embedded minimal disk $\Sigma$ in a half-space with $\partial \Sigma \subset \partial B_{2 r_{0}}$ : The components of $B_{r_{0}} \cap \Sigma$ intersecting $B_{\epsilon r_{0}}$ are graphs.

Using the minimal surface equation and that $\Sigma^{\prime}$ has points close to a plane, it is not hard to see that, for $\epsilon>0$ sufficiently small, (18.4) is equivalent to the statement that $\Sigma^{\prime}$ is a graph over the plane $\left\{x_{3}=0\right\}$.

An embedded minimal surface $\Sigma$ which is as in Theorem 18.3 is said to satisfy the $\left(\epsilon, r_{0}\right)$-effective one-sided Reifenberg condition; cf. appendix A of [CM6] and the appendix of $[\mathbf{C h C 1}]$. We will often refer to Theorem 18.3 as the one-sided curvature estimate.

Note that the assumption in Theorem 18.3 that $\Sigma$ is simply connected is crucial as can be seen from the example of a rescaled catenoid. The catenoid is the minimal surface in $\mathbf{R}^{3}$ given by

$$
(\cosh s \cos t, \cosh s \sin t, s)
$$




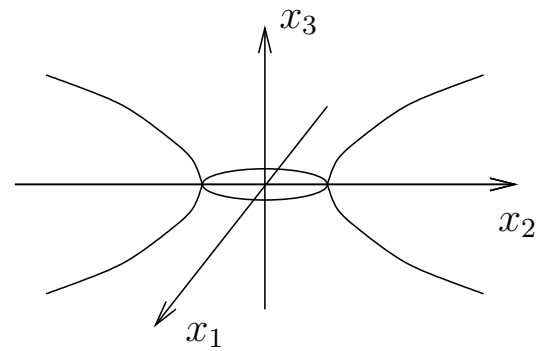

Figure 7. The catenoid given by revolving $x_{1}=\cosh x_{3}$ around the $x_{3}$-axis.

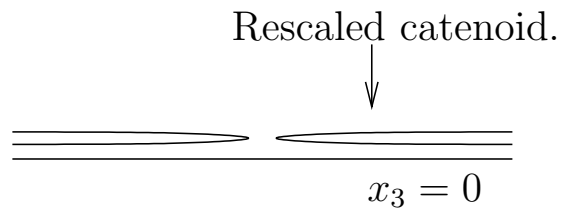

Figure 8. Rescaling the catenoid shows that simply connected (and embedded) is needed in the one-sided curvature estimate.

where $s, t \in \mathbf{R}$; see fig. 7. Under rescalings this converges (with multiplicity two) to the flat plane; see fig. 8. Likewise, by considering the universal cover of the catenoid, one sees that embedded, and not just immersed, is needed in Theorem 18.3 .

As an almost immediate consequence of Theorem 18.3 and a simple barrier argument we get that if in a ball two embedded minimal disks come close to each other near the center of the ball then each of the disks are graphs. Precisely, this is the following:

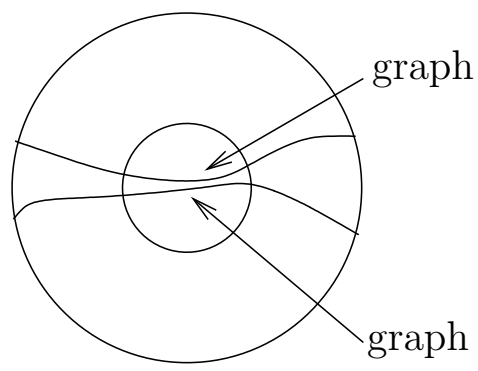

Figure 9. Corollary 18.4: Two sufficiently close components of an embedded minimal disk must each be a graph.

Corollary 18.4. (Corollary 0.4 in [CM6]). See fig. 9. There exist $c>1$, $\epsilon>0$ so that the following holds:

Let $\Sigma_{1}$ and $\Sigma_{2} \subset B_{c r_{0}} \subset \mathbf{R}^{3}$ be disjoint embedded minimal surfaces with $\partial \Sigma_{i} \subset$ $\partial B_{c r_{0}}$ and $B_{\epsilon r_{0}} \cap \Sigma_{i} \neq \emptyset$. If $\Sigma_{1}$ is a disk, then for all components $\Sigma_{1}^{\prime}$ of $B_{r_{0}} \cap \Sigma_{1}$ which intersect $B_{\epsilon r_{0}}$

$$
\sup _{\Sigma_{1}^{\prime}}|A|^{2} \leq r_{0}^{-2}
$$

This estimate has also been useful in the global theory of minimal surfaces, cf. [CM9], [CM22], and [MeRo]. It would be very interesting to find an intrinsic version of it (i.e., for intrinsic balls on one side of a plane): 
QUESTION 3. It would be very useful to prove an intrinsic version of the onesided curvature estimate. Namely, does Theorem 18.3 hold when $\Sigma$ is an intrinsic ball (in an embedded minimal disk)? If true, then this would likely have important consequences for proving properness of embedded minimal disks.

One of the topics that we have suppressed is what we call "properness" of the limit. Basically, this is proving that we get a foliation in the limit or, equivalently, that the points of $\mathcal{S}$ cannot be isolated. This is the one place where the assumption $R_{i} \rightarrow \infty$ is used in an essential way; see [CM18] for a nonproper limit when $R_{i}$ does not go to $\infty$.

QUESTION 4. Suppose that a sequence of embedded minimal planar domains $\Sigma_{i} \subset B_{1} \subset \mathbf{R}^{3}$ with $\partial \Sigma_{i} \subset \partial B_{1}$ converges away from 0 to a minimal lamination $\mathcal{L}^{\prime}$ of $B_{1} \backslash\{0\}$. Does $\mathcal{L}^{\prime}$ extend to a smooth lamination of $B_{1}$ ? In other words, is 0 a removable singularity? An example constructed in [CM18] shows that this need not be the case when the $\Sigma_{i}$ 's are disks. It would be interesting to find non-disk examples (cf. $[\mathbf{K a}],[\mathbf{T r}])$.

\section{Global theory of minimal surfaces in $\mathbf{R}^{3}$}

Recent years have seen breakthroughs on many long-standing problems in the global theory of minimal surfaces in $\mathbf{R}^{3}$. This is an enormous subject and, rather than give a comprehensive treatment, we will mention a few important results which fit well with the theme of this survey. Throughout this section, $\Sigma$ will be a complete properly embedded minimal surface in $\mathbf{R}^{3}$ (recall that properness here means that the intersection of $\Sigma$ with any compact subset of $\mathbf{R}^{3}$ is compact).

We say that $\Sigma$ has finite topology if it is homeomorphic to a closed Riemann surface with a finite number of punctures; the genus of $\Sigma$ is then the genus of this Riemann surface and the number of punctures is the number of ends. It follows that a neighborhood of each puncture corresponds to a properly embedded annular end of $\Sigma$. Perhaps surprisingly at first, the more restrictive case is when $\Sigma$ has more than one end. The reason for this is that a barrier argument gives a stable minimal surface between any pair of ends. This stable surface is then asymptotic to a plane (or catenoid), essentially forcing each end to live in a half-space. Using this restriction, P. Collin proved:

THEOREM 19.1. [Co] Each end of a complete properly embedded minimal surface with finite topology and at least two ends is asymptotic to a plane or catenoid.

In particular, outside some compact set, $\Sigma$ is given by a finite collection of disjoint graphs over a common plane (and has finite total curvature). See [CM21] for a proof of Theorem 19.1 using the one-sided curvature estimate.

When $\Sigma$ has only one end (e.g., for the helicoid), it need not have finite total curvature so the situation is more delicate. However, the regularity results of the previous section can be applied. For example, if $\Sigma$ is a (non-planar) embedded minimal disk, then we get a multi-valued graph structure away from a "one-dimensional singular set." Using Theorems 18.1 and 18.3, W. Meeks and H. Rosenberg proved the uniqueness of the helicoid:

THEOREM 19.2. [MeRo] The plane and helicoid are the only complete properly embedded simply-connected minimal surfaces in $\mathbf{R}^{3}$. 
This uniqueness should have many applications. Recall that if we take a sequence of rescalings of the helicoid, then the singular set $\mathcal{S}$ for the convergence is the vertical axis perpendicular to the leaves of the foliation. In [Me1], W. Meeks used this fact together with the uniqueness of the helicoid to prove that the singular set $\mathcal{S}$ in Theorem 18.1 is always a straight line perpendicular to the foliation. Recently, W. Meeks and M. Weber have constructed a local example (i.e., a sequence of embedded minimal disks in a unit ball) where $\mathcal{S}$ is a circle.

Properness is an important ingredient in many of these results and it is not known to what extent this assumption can be relaxed. In [Na], N. Nadirashvili constructed complete non-proper minimal immersions (in fact, contained in a ball). It would be interesting to know whether this is possible for embeddings:

QUESTION 5. Suppose that $\Sigma \subset \mathbf{R}^{3}$ is a complete embedded minimal surface with finite topology. Does $\Sigma$ have to be proper?

We have not even touched on the case where $\Sigma$ has infinite topology (e.g., when $\Sigma$ is one of the Riemann examples). This is an area of much current research, see [CM5], the work of Meeks, J. Perez and A. Ros, [MePRs1], [MePRs2], and [MePRs3], the survey $[\mathbf{M e P}]$ and references therein.

We close this section with a local analog of the two-ended case. Namely, in [CM9], we proved that any embedded minimal annulus in a ball (with boundary in the boundary of the ball and) with a small neck can be decomposed by a simple closed geodesic into two graphical sub-annuli. Moreover, we gave a sharp bound for the length of this closed geodesic in terms of the separation (or height) between the graphical sub-annuli. This serves to illustrate our "pair of pants" decomposition from [CM5] in the special case where the embedded minimal planar domain is an annulus (we will not touch on this further here). The catenoid

$$
\left\{x_{1}^{2}+x_{2}^{2}=\cosh ^{2} x_{3}\right\}
$$

is the prime example of an embedded minimal annulus.

The precise statement of this decomposition for annuli is:

Theorem 19.3. [CM9] There exist $\epsilon>0, C_{1}, C_{2}, C_{3}>1$ so: If $\Sigma \subset B_{R} \subset \mathbf{R}^{3}$ is an embedded minimal annulus with $\partial \Sigma \subset \partial B_{R}$ and $\pi_{1}\left(B_{\epsilon R} \cap \Sigma\right) \neq 0$, then there exists a simple closed geodesic $\gamma \subset \Sigma$ of length $\ell$ so that:

- The curve $\gamma$ splits the connected component of $B_{R / C_{1}} \cap \Sigma$ containing it into two annuli $\Sigma^{+}, \Sigma^{-}$each with $\int|A|^{2} \leq 5 \pi$.

- Furthermore, $\Sigma^{ \pm} \backslash \mathcal{T}_{C_{2} \ell}(\gamma)$ are graphs with gradient $\leq 1$.

- Finally, $\ell \log (R / \ell) \leq C_{3} h$ where the separation $h$ is given by

$$
h=\min _{x_{ \pm} \in \partial B_{R / C_{1}} \cap \Sigma^{ \pm}}\left|x_{+}-x_{-}\right| .
$$

Here $\mathcal{T}_{s}(S) \subset \Sigma$ denotes the intrinsic $s$-tubular neighborhood of a subset $S \subset \Sigma$.

\section{Part 4. Constructing minimal surfaces and applications}

Thus far, we have mainly dealt with regularity and a priori estimates but have ignored questions of existence. In this part we surveys some of the most useful existence results for minimal surfaces and gives an application to Ricci flow. Section 20 recalls the classical Weierstrass representation, including a few modern applications, 
and the Kapouleas desingularization method. Section 21 deals with producing area minimizing surfaces (whether for fixed boundary, fixed homotopy class, etc.) and questions of embeddedness. The next section discusses unstable (hence not minimizing) surfaces and the corresponding questions for geodesics, concentrating on whether the Morse index can be bounded uniformly. Section 23 recalls the minmax construction for producing unstable minimal surfaces and, in particular, doing so while controlling the topology and guaranteeing embeddedness. Finally, Section 24 discusses a recent application of min-max surfaces to bound the extinction time for Ricci flow, answering a question of Perelman.

\section{The Weierstrass representation}

The classical Weierstrass representation (see $[\mathbf{H o K}]$ or $[\mathbf{O s}]$ ) takes holomorphic data (a Riemann surface, a meromorphic function, and a holomorphic one-form) and associates a minimal surface in $\mathbf{R}^{3}$. To be precise, given a Riemann surface $\Omega$, a meromorphic function $g$ on $\Omega$, and a holomorphic one-form $\phi$ on $\Omega$, then we get a (branched) conformal minimal immersion $F: \Omega \rightarrow \mathbf{R}^{3}$ by

$$
F(z)=\operatorname{Re} \int_{\zeta \in \gamma_{z_{0}, z}}\left(\frac{1}{2}\left(g^{-1}(\zeta)-g(\zeta)\right), \frac{i}{2}\left(g^{-1}(\zeta)+g(\zeta)\right), 1\right) \phi(\zeta) .
$$

Here $z_{0} \in \Omega$ is a fixed base point and the integration is along a path $\gamma_{z_{0}, z}$ from $z_{0}$ to $z$. The choice of $z_{0}$ changes $F$ by adding a constant. In general, the map $F$ may depend on the choice of path (and hence may not be well-defined); this is known as "the period problem" (see M. Weber and M. Wolf, [WeWo], for the latest developments). However, when $g$ has no zeros or poles and $\Omega$ is simply connected, then $F(z)$ does not depend on the choice of path $\gamma_{z_{0}, z}$.

Two standard constructions of minimal surfaces from Weierstrass data are

$$
\begin{aligned}
& g(z)=z, \phi(z)=d z / z, \Omega=\mathbf{C} \backslash\{0\} \text { giving a catenoid } \\
& g(z)=\mathrm{e}^{i z}, \phi(z)=d z, \Omega=\mathbf{C} \text { giving a helicoid }
\end{aligned}
$$

The Weierstrass representation is particularly useful for constructing immersed minimal surfaces. For example, in $[\mathbf{N a}]$, Nadirashvili used it to construct a complete immersed minimal surface in the unit ball in $\mathbf{R}^{3}$ (see also [JXa] for the case of a slab). In particular, Nadirashvili's surface is not proper, i.e., the intersections with compact sets are not necessarily compact.

Typically, it is rather difficult to prove that the resulting immersion is an embedding (i.e., is 1-1), although there are some interesting cases where this can be done. The first modern example was [HoMe] where D. Hoffman and Meeks proved that the surface constructed by Costa was embedded; this was the first new complete finite topology properly embedded minimal surface discovered since the classical catenoid, helicoid, and plane. This led to the discovery of many more such surfaces (see $[\mathbf{H o K}]$ and $[\mathbf{R o}]$ for more discussion).

Very recently in [HoWeWo], Hoffman, Weber, and Wolf have used the Weierstrass representation to construct a genus one properly embedded minimal surface asymptotic to the helicoid. They construct this "genus one helicoid" as the limit of a continuous one-parameter family of screw-motion invariant minimal surfaces-also asymptotic to the helicoid-that have genus equal to one in the quotient. 
In [CM18], we used the Weierstrass representation to construct a sequence of embedded minimal disks

$$
\Sigma_{i} \subset B_{1}=B_{1}(0) \subset \mathbf{R}^{3}
$$

with $\partial \Sigma_{i} \subset \partial B_{1}$ where the curvatures blow up only at 0 and $\Sigma_{i} \backslash\left\{x_{3}\right.$-axis $\}$ consists of two multi-valued graphs for each $i$. Furthermore, $\Sigma_{i} \backslash\left\{x_{3}=0\right\}$ converges to two embedded minimal disks $\Sigma^{-} \subset\left\{x_{3}<0\right\}$ and $\Sigma^{+} \subset\left\{x_{3}>0\right\}$ each of which spirals into $\left\{x_{3}=0\right\}$ and thus is not proper. (This should be contrasted with Theorem 18.1 where the complete limits are planes and hence proper.)

N. Kapouleas has developed another method to construct complete embedded minimal surfaces with finite total curvature. For instance, in $[\mathbf{K a}]$, he shows that (most) collections of coaxial catenoids and planes can be desingularized to get complete embedded minimal surfaces with finite total curvature. The Costa surface above had genus one and three ends (that is to say, it is homeomorphic to a torus with three punctures). In the Kapouleas construction, one could start with a plane and catenoid intersecting in a circle and then desingularize this circle using suitably scaled and bent Scherk surfaces to get a finite genus embedded surface with three ends. (This desingularization process adds handles, i.e., increases the genus.) In this manner, Kapouleas gets an enormous number of new examples; see also the gluing construction of S.D. Yang, $[\mathbf{Y}]$, which uses catenoid necks to glue together nearby minimal surfaces.

\section{Area-minimizing surfaces}

Perhaps the most natural way to construct minimal surfaces is to look for ones which minimize area, e.g., with fixed boundary, or in a homotopy class, etc. This has the advantage that often it is possible to show that the resulting surface is embedded. We mention a few results along these lines.

The first embeddedness result, due to Meeks and Yau, shows that if the boundary curve is embedded and lies on the boundary of a smooth mean convex set (and it is null-homotopic in this set), then it bounds an embedded least area disk.

TheOREM 21.1. [MeYa1] Let $M^{3}$ be a compact Riemannian three-manifold whose boundary is mean convex and let $\gamma$ be a simple closed curve in $\partial M$ which is null-homotopic in $M$; then $\gamma$ is bounded by a least area disk and any such least area disk is properly embedded.

Note that some restriction on the boundary curve $\gamma$ is certainly necessary. For instance, if the boundary curve was knotted (e.g., the trefoil), then it could not be spanned by any embedded disk (minimal or otherwise). Prior to the work of Meeks and Yau, embeddedness was known for extremal boundary curves in $\mathbf{R}^{3}$ with small total curvature by the work of R. Gulliver and J. Spruck [GuSp]; see chapter 4 in [CM1] for other results and further discussion.

If we instead fix a homotopy class of maps, then the two fundamental existence results are due to Sacks-Uhlenbeck and Schoen-Yau (with embeddedness proven by Meeks-Yau and Freedman-Hass-Scott, respectively):

Theorem 21.2. [SaUh], [MeYa2] Given $M^{3}$, there exist conformal (stable) minimal immersions $u_{1}, \ldots, u_{m}: \mathbf{S}^{2} \rightarrow M$ which generate $\pi_{2}(M)$ as a $\mathbf{Z}\left[\pi_{1}(M)\right]$ module. Furthermore,

- If $u: \mathbf{S}^{2} \rightarrow M$ and $[u]_{\pi_{2}} \neq 0$, then Area $(u) \geq \min _{i} \operatorname{Area}\left(u_{i}\right)$. 
- Each $u_{i}$ is either an embedding or a 2-1 map onto an embedded 2-sided $\mathbf{R} P^{2}$.

TheOREM 21.3. [ScYa2], [FHS] If $\Sigma^{2}$ is a closed surface with genus $g>0$ and $i_{0}: \Sigma \rightarrow M^{3}$ is an embedding which induces an injective map on $\pi_{1}$, then there is a least area embedding with the same action on $\pi_{1}$.

In [MeSiYa], Meeks, Simon, and Yau find an embedded sphere minimizing area in an isotopy class in a closed 3-manifold.

We end this section by mentioning two applications of Theorem 21.3. First, in [CM20], we showed that any topological 3-manifold $M$ had an open set of metrics so that, for each such metric, there was a sequence of embedded minimal tori whose area went to infinity. In $[\mathbf{D e}], \mathrm{B}$. Dean showed that this was true for every genus $g \geq 1$. This leaves an obvious interesting question:

Question 6. Given a topological 3-manifold $M$, does there exist an open set of metrics which have embedded minimal spheres with arbitrarily large area?

It would be interesting to answer this question even when the minimal spheres are stable (the examples constructed in [CM20] and [De] were all locally minimizing and hence also stable)

\section{Index bounds for geodesics and minimal surfaces}

The minimal surfaces discussed in the previous section were all stable and in fact locally area minimizing. This is a very special and strong property of a minimal surface. In general, like, for instance the helicoid and the catenoid, most minimal surfaces are not stable but have non-zero index. In this section we will discuss the Morse index of simple closed geodesics on surfaces and of embedded minimal surfaces in 3-manifolds. First let us discuss the situation of simple closed geodesics in surfaces.

Let $M^{2}$ be a closed orientable surface with curvature $K$ and $\gamma \subset M$ a closed geodesic. The Morse index of $\gamma$ is the index of the critical point $\gamma$ for the length functional, i.e., the number of negative eigenvalues (counted with multiplicity) of the second derivative of length (throughout curves will always be in $H^{1}$ ). Since the second derivative of length at $\gamma$ in the direction of a normal variation $u \mathrm{n}$ is

$$
-\int_{\gamma} u L_{\gamma} u
$$

where

$$
L_{\gamma} u=u^{\prime \prime}+K u,
$$

the Morse index is the number of negative eigenvalues of $L_{\gamma}$. (By convention, an eigenfunction $\phi$ with eigenvalue $\lambda$ of $L_{\gamma}$ is a solution of $L_{\gamma} \phi+\lambda \phi=0$.) Note that if $\lambda=0$, then $\phi$ (or $\phi \mathrm{n}$ ) is a (normal) Jacobi field. $\gamma$ is stable if the index is zero. The index of a noncompact geodesic is the dimension of a maximal vector space of compactly supported variations for which the second derivative of length is negative definite. We also say that such a geodesic is stable if the index is 0 .

As the following result shows then it turns out that in general there are no Morse index bounds for simple closed geodesics on surfaces. 
THEOREM 22.1. [CH1]. On any $M^{2}$, there exists a metric with a geodesic lamination with infinitely many unstable leaves. Moreover, there is such a metric with simple closed geodesics of arbitrary high Morse index.

A codimension one lamination on a surface $M^{2}$ is a collection $\mathcal{L}$ of smooth disjoint curves (called leaves) such that $\cup_{\ell \in \mathcal{L}} \ell$ is closed. Moreover, for each $x \in M$ there exists an open neighborhood $U$ of $x$ and a $C^{0}$ coordinate chart, $(U, \Phi)$, with $\Phi(U) \subset \mathbf{R}^{2}$ so that in these coordinates the leaves in $\mathcal{L}$ pass through $\Phi(U)$ in slices of the form $(\mathbf{R} \times\{t\}) \cap \Phi(U)$. A foliation is a lamination for which the union of the leaves is all of $M$ and a geodesic lamination is a lamination whose leaves are geodesics.

Similarly, to the geodesic case, for an immersed minimal surface $\Sigma$ in a $3-$ manifold $M$, we set

$$
L_{\Sigma} \phi=\Delta_{\Sigma} \phi+|A|^{2} \phi+\operatorname{Ric}_{M}(\mathrm{n}, \mathrm{n}) \phi .
$$

(Note that by the second variational formula (see, for instance, section 1.7 of [CM1]), then

$$
\frac{\partial^{2}}{\partial r^{2}} \underset{r=0}{\operatorname{Area}}\left(\Sigma_{r}\right)=-\int_{\Sigma} \phi L_{\Sigma} \phi,
$$

where $\Sigma_{r}=\left\{x+r \phi(x) \mathrm{n}_{\Sigma}(x) \mid x \in \Sigma\right\}$.) Recall also that by definition the index of a minimal surface $\Sigma$ is the number of negative eigenvalues (counted with multiplicity) of $L_{\Sigma}$. (A function $\eta$ is an eigenfunction of $L_{\Sigma}$ with eigenvalue $\lambda$ if $L_{\Sigma} \eta+\lambda \eta=0$.) Thus in particular, since $\Sigma$ is assumed to be closed, the index is always finite.

Theorem 22.1 was proven by first constructing a metric on the disk with convex boundary having no Morse index bounds and then completing the metric to a metric on the given $M^{2}$. By taking the product of this metric on the disk with a circle we get, on a solid torus, a metric with convex boundary and without Morse index bounds for embedded minimal tori, and with a minimal lamination with infinitely many unstable leaves. By completing this metric we get:

THEOREM 22.2. [CH2] On any $M^{3}$, there exists a metric with a minimal lamination with infinitely many unstable leaves. Moreover, there is such a metric with embedded minimal tori of arbitrary high Morse index.

By construction the embedded minimal tori in Theorem 22.2 and the leaves of the lamination can be taken to be totally geodesic.

We will equip the space of metrics on a given manifold with the $C^{\infty}$-topology. A subset of the set of metrics on the manifold is said to be residual if it is a countable intersection of open dense subsets. A metric on a surface is bumpy if each closed geodesic is a nondegenerate critical point, i.e., $L_{\gamma} u=0$ implies $u \equiv 0$. It follows from results of Abraham and Anosov that bumpy metrics are generic; that is the set of bumpy metrics contain a residual set.

To check that any given metric is bumpy is virtually impossible; however it seems that the metric in Theorem 22.1 can be chosen to be bumpy. Thus it seems unlikely that a bumpy metric is enough to ensure a bound for the Morse index of simple closed geodesics on $M^{2}$. What is needed is a nondegeneracy condition for noncompact simple geodesics, rather than one for closed geodesics; cf. [CH2], [CH3].

In $[\mathbf{H a N o R u}]$ examples were given of metrics on any $M^{3}$ that have embedded minimal spheres without bounds and in [CD2] the following was shown: For any 
3-manifold $M^{3}$ and any nonnegative integer $\mathbf{g}$, there are examples of metrics on $M$ each of which has a sequence of embedded minimal surfaces of genus $\mathbf{g}$ and without Morse index bounds. On any spherical space form $\mathbf{S}^{3} / \Gamma[\mathbf{C D 2}]$ constructed such a metric with positive scalar curvature. More generally [CD2] constructed such a metric with Scal $>0$ (and such surfaces) on any 3-manifold which carries a metric with Scal $>0$. In all but one of the examples in [CD2] the Hausdorff limit is a singular minimal lamination. The singularities being in each case exactly two points lying on a closed leaf (the leaf is a strictly stable sphere).

[CD2] used in part ideas of Hass-Norbury-Rubinstein [HaNoRu]. As in $[$ HaNoRu], but unlike the examples in [CH1], the surfaces in [CD2] have no uniform curvature bounds. In fact, it follows easily (see appendix B of [CM4]) that if $\Sigma_{i} \subset M^{3}$ is a sequence of embedded minimal surfaces with uniformly bounded curvatures, then a subsequence converges to a smooth lamination. Moreover, with the right notion of being generic, the following seems likely (by [CH1] bumpy is not the right generic notion):

Question 7. Let $M^{3}$ be a closed 3-manifold with a generic metric and $\Sigma_{i} \subset M$ a sequence of embedded minimal surfaces of a given genus. If any limit of the $\Sigma_{i}$ 's is a smooth (minimal) lamination, then the sequence $\Sigma_{i}$ has a uniform Morse index bound.

A codimension one lamination of $M^{3}$ is a collection $\mathcal{L}$ of smooth disjoint connected surfaces (called leaves) such that $\cup_{\Lambda \in \mathcal{L}} \Lambda$ is closed. Moreover, for each $x \in M$ there exists an open neighborhood $U$ of $x$ and a local coordinate chart, $(U, \Phi)$, with $\Phi(U) \subset \mathbf{R}^{3}$ such that in these coordinates the leaves in $\mathcal{L}$ pass through the chart in slices of the form $\left(\mathbf{R}^{2} \times\{t\}\right) \cap \Phi(U)$.

A lamination is said to be minimal if the leaves are (smooth) minimal surfaces. If the union of the leaves is all of $M$, then it is a foliation.

There are two results that support this question. The first concerns the corresponding question in one dimension less (that is for geodesics on surfaces); see [CH2], [CH3]. The second concerns the question for 3-manifolds with positive scalar curvature. However, there are examples where the limit is not smooth; see [CD2].

Finally, we refer to $[\mathbf{C H 2}]$ and [CH3] for further discussion of Morse index bounds for geodesics including some positive results about when one has such bounds.

\section{The min-max construction of minimal surfaces}

Variational arguments can also be used to construct higher index (i.e., nonminimizing) minimal surfaces using the topology of the space of surfaces. There are two basic approaches:

- Applying Morse theory to the energy functional on the space of maps from a fixed surface $\Sigma$ to $M$.

- Doing a min-max argument over families of (topologically non-trivial) sweepouts of $M$.

The first approach has the advantage that the topological type of the minimal surface is easily fixed; however, the second approach has been more successful at producing embedded minimal surfaces. We will highlight a few key results below but refer to [CD1] for a thorough treatment. 
Unfortunately, one cannot directly apply Morse theory to the energy functional on the space of maps from a fixed surface because of a lack of compactness (the Palais-Smale Condition $\mathrm{C}$ does not hold). To get around this difficulty, [SaUh] introduce a family of perturbed energy functionals which do satisfy Condition $\mathrm{C}$ and then obtain minimal surfaces as limits of critical points for the perturbed problems:

TheOREM 23.1. [SaUh] If $\pi_{k}(M) \neq 0$ for some $k>1$, then there exists a branched immersed minimal 2-sphere in $M$ (for any metric).

This was sharpened somewhat by [MiMo] (showing that the index of the minimal sphere was at most $k-2$ ), who used it to prove a generalization of the sphere theorem. See $[\mathbf{J o}]$ and $[\mathbf{S t}]$ for approaches which avoid using the perturbed functionals and $[\mathbf{F r}]$ for a generalization to a free boundary problem.

The basic idea of constructing minimal surfaces via min-max arguments and sweep-outs goes back to Birkhoff, who developed it to construct simple closed geodesics on spheres. In particular, when $M$ is a topological 2-sphere, we can find a 1-parameter family of curves starting and ending at point curves so that the induced map $F: \mathbf{S}^{2} \rightarrow \mathbf{S}^{2}$ (see fig. 10) has nonzero degree. The min-max argument produces a nontrivial closed geodesic of length less than or equal to the longest curve in the initial one-parameter family. A curve shortening argument gives that the geodesic obtained in this way is simple.

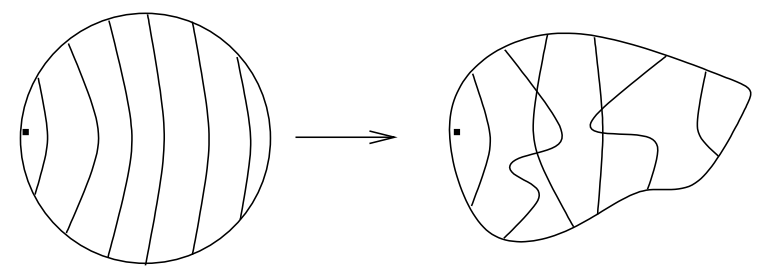

Figure 10. A 1-parameter family of curves on a 2 -sphere which induces a $\operatorname{map} F: \mathbf{S}^{2} \rightarrow \mathbf{S}^{2}$ of degree 1 .

In $[\mathbf{P i}]$, J. Pitts applied a similar argument and geometric measure theory to get that every closed Riemannian three manifold has an embedded minimal surface (his argument was for dimensions up to seven), but he did not estimate the genus of the resulting surface. Finally, F. Smith (under the direction of L. Simon) proved (see $[$ CD1]):

THEOREM 23.2. [Sm] Every metric on a topological 3-sphere $M$ admits an embedded minimal 2-sphere.

The main new contribution of Smith was to control the topological type of the resulting minimal surface while keeping it embedded; see also Pitts and Rubinstein, [PiRu], for some generalizations.

\section{An application of min-max surfaces to Ricci flow}

We will in this section give an application of the min-max construction of minimal surfaces to the Ricci flow. This application is that on $\mathbf{S}^{3}$ starting at any 
given metric the Ricci flow becomes extinct in finite time. The treatment here follows [CM19] and was inspired by a question of Perelman; see [CM19], [Pe3]. (See also paragraph 4.4 of $[\mathbf{P e 2}]$ for the precise definition of extinction time in the case that surgery occurs.)

Throughout this section we let $M^{3}$ be the 3 -sphere and let $g(t)$ be a oneparameter family of metrics on $M$ evolving by the Ricci flow, so

$$
\partial_{t} g=-2 \operatorname{Ric}_{M_{t}} .
$$

Since $\pi_{3}(M)=\mathbf{Z}$, it follows from suspension, as in lemma 3 of [MiMo], that the space of maps from $\mathbf{S}^{2}$ to $M$ is not simply connected.

Fix a continuous map

$$
\beta:[0,1] \rightarrow C^{0} \cap L_{1}^{2}\left(\mathbf{S}^{2}, M\right)
$$

where $\beta(0)$ and $\beta(1)$ are constant maps so that $\beta$ is in the nontrivial homotopy class $[\beta]$. We define the width $W=W(g,[\beta])$ by

$$
W(g)=\min _{\gamma \in[\beta]} \max _{s \in[0,1]} \operatorname{Energy}(\gamma(s)) .
$$

One could equivalently define the width using the area rather than the energy, but the energy is somewhat easier to work with. As for the Plateau problem, this equivalence follows using the uniformization theorem and the inequality $\operatorname{Area}(u) \leq$ $\operatorname{Energy}(u)$ (with equality when $u$ is a branched conformal map); cf. lemma 4.12 in [CM1].

The next theorem gives an upper bound for the derivative of $W(g(t))$ under the Ricci flow which forces the solution $g(t)$ to become extinct in finite time.

TheOREm 24.1. ([CM19] and cf. [Pe3]). Let $M^{3}$ be the 3-sphere equipped with a Riemannian metric $g=g(0)$. Under the Ricci flow, the width $W(g(t))$ satisfies

$$
\frac{d}{d t} W(g(t)) \leq-4 \pi+\frac{3}{4(t+C)} W(g(t)),
$$

in the sense of the limsup of forward difference quotients. Hence, $g(t)$ must become extinct in finite time.

Suppose that $\Sigma \subset M$ is a closed immersed surface (not necessarily minimal), then using (24.1) an easy calculation gives (cf. page 38-41 of [Ha3])

$$
\frac{d}{d t} \operatorname{Area}_{t=0}(\Sigma)=-\int_{\Sigma}\left[R-\operatorname{Ric}_{M}(\mathrm{n}, \mathrm{n})\right] .
$$

If $\Sigma$ is also minimal, then

$$
\begin{aligned}
\frac{d}{d t} \operatorname{Area}_{t=0}(\Sigma) & =-2 \int_{\Sigma} \mathrm{K}_{\Sigma}-\int_{\Sigma}\left[|A|^{2}+\operatorname{Ric}_{M}(\mathrm{n}, \mathrm{n})\right] \\
& =-\int_{\Sigma} K_{\Sigma}-\frac{1}{2} \int_{\Sigma}\left[|A|^{2}+R\right]
\end{aligned}
$$

Here $\mathrm{K}_{\Sigma}$ is the (intrinsic) curvature of $\Sigma, \mathrm{n}$ is a unit normal for $\Sigma$ (our $\Sigma$ 's below will be $\mathbf{S}^{2}$ 's and hence have a well-defined unit normal), $A$ is the second fundamental form of $\Sigma$ so that $|A|^{2}$ is the sum of the squares of the principal curvatures, $\operatorname{Ric}_{M}$ is the Ricci curvature of $M$, and $R$ is the scalar curvature of $M$. (The curvature is 
normalized so that on the unit $\mathbf{S}^{3}$ the Ricci curvature is 2 and the scalar curvature is 6.) To get (24.6), we used that by the Gauss equations and minimality of $\Sigma$

$$
\mathrm{K}_{\Sigma}=\mathrm{K}_{M}-\frac{1}{2}|A|^{2},
$$

where $\mathrm{K}_{M}$ is the sectional curvature of $M$ on the two-plane tangent to $\Sigma$.

Our first lemma gives an upper bound for the rate of change of area of minimal 2-spheres.

LEMma 24.2. If $\Sigma \subset M^{3}$ is a branched minimal immersion of the 2-sphere, then

$$
\frac{d}{d t}{ }_{t=0} \operatorname{Area}_{g(t)}(\Sigma) \leq-4 \pi-\frac{\operatorname{Area}_{g(0)}(\Sigma)}{2} \min _{M} R(0) .
$$

Proof. Let $\left\{p_{i}\right\}$ be the set of branch points of $\Sigma$ and $b_{i}>0$ the order of branching at $p_{i}$. By $(24.6)$

$$
\frac{d}{d t}{ }_{t=0} \operatorname{Area}_{g(t)}(\Sigma) \leq-\int_{\Sigma} K_{\Sigma}-\frac{1}{2} \int_{\Sigma} R=-4 \pi-2 \pi \sum b_{i}-\frac{1}{2} \int_{\Sigma} R,
$$

where the equality used the Gauss-Bonnet theorem with branch points.

We need to recall a result on harmonic maps which gives the existence of minimal spheres realizing the width $W(g)$. The results of Sacks and Uhlenbeck give the harmonic maps but potentially allow some loss of energy. This energy loss was ruled out by Siu and Yau (using also arguments of Meeks and Yau), see Chapter VIII in [ScYa3]. For our purposes, the most convenient statement of this is given in theorem 4.2.1 of [Jo]. (The bound for the index is not stated explicitly in $[\mathbf{J o}]$ but follows immediately as in [MiMo].)

Proposition 24.3. Given a metric $g$ on $M$ and a nontrivial $[\beta] \in \pi_{1}\left(C^{0} \cap\right.$ $\left.L_{1}^{2}\left(\mathbf{S}^{2}, M\right)\right)$, there exists a sequence of sweep-outs $\gamma^{j}:[0,1] \rightarrow C^{0} \cap L_{1}^{2}\left(\mathbf{S}^{2}, M\right)$ with $\gamma^{j} \in[\beta]$ so that

$$
W(g)=\lim _{j \rightarrow \infty} \max _{s \in[0,1]} \operatorname{Energy}\left(\gamma_{s}^{j}\right) .
$$

Furthermore, there exist $s_{j} \in[0,1]$ and branched conformal minimal immersions $u_{0}, \ldots, u_{m}: \mathbf{S}^{2} \rightarrow M$ with index at most one so that, as $j \rightarrow \infty$, the maps $\gamma_{s_{j}}^{j}$ converge to $u_{0}$ weakly in $L_{1}^{2}$ and uniformly on compact subsets of $\mathbf{S}^{2} \backslash\left\{x_{1}, \ldots, x_{k}\right\}$, and

$$
W(g)=\sum_{i=0}^{m} \operatorname{Energy}\left(u_{i}\right)=\lim _{j \rightarrow \infty} \operatorname{Energy}\left(\gamma_{s_{j}}^{j}\right) .
$$

Finally, for each $i>0$, there exists a point $x_{k_{i}}$ and a sequence of conformal dilations $D_{i, j}: \mathbf{S}^{2} \rightarrow \mathbf{S}^{2}$ about $x_{k_{i}}$ so that the maps $\gamma_{s_{j}}^{j} \circ D_{i, j}$ converge to $u_{i}$.

We will also need a standard additional property for the min-max sequence of sweep-outs $\gamma^{j}$ of Proposition 24.3 which can be achieved by modifying the sequence as in section 4 of [CD1] (cf. proposition 4.1 on page 85 in [CD1]). Loosely speaking this is the property that any subsequence $\gamma_{s_{k}}^{k}$ with energy converging to $W(g)$ converges (after possibly going to a further subsequence) to the union of branched immersed minimal 2-spheres each with index at most one. Precisely this is that we 
can choose $\gamma^{j}$ so that: Given $\epsilon>0$, there exist $J$ and $\delta>0$ (both depending on $g$ and $\gamma^{j}$ ) so that if $j>J$ and

$$
\operatorname{Energy}\left(\gamma_{s}^{j}\right)>W(g)-\delta,
$$

then there is a collection of branched minimal 2-spheres $\left\{\Sigma_{i}\right\}$ each of index at most one and with

$$
\operatorname{dist}\left(\gamma_{s}^{j}, \cup_{i} \Sigma_{i}\right)<\epsilon .
$$

Here, the distance means varifold distance (see, for instance, section 4 of [CD1]). Below we will use that, as an immediate consequence of (24.13), if $F$ is a quadratic form on $M$ and $\Gamma$ denotes $\gamma_{s}^{j}$, then

$$
\left|\int_{\Gamma}\left[\operatorname{Tr}(F)-F\left(\mathrm{n}_{\Gamma}, \mathrm{n}_{\Gamma}\right)\right]-\sum_{i} \int_{\Sigma_{i}}\left[\operatorname{Tr}(F)-F\left(\mathrm{n}_{\Sigma_{i}}, \mathrm{n}_{\Sigma_{i}}\right)\right]\right|<C \epsilon\|F\|_{C^{1}} \operatorname{Area}(\Gamma) .
$$

Proof. (of Theorem 24.1) Fix a time $\tau$. Below $\tilde{C}$ denotes a constant depending only on $\tau$ but will be allowed to change from line to line. Let $\gamma^{j}(\tau)$ be the sequence of sweep-outs for the metric $g(\tau)$ given by Proposition 24.3. We will use the sweepout at time $\tau$ as a comparison to get an upper bound for the width at times $t>\tau$. The key for this is the following claim:

Given $\epsilon>0$, there exist $J$ and $\bar{h}>0$ so that if $j>J$ and $0<h<\bar{h}$, then

$$
\text { Area }_{g(\tau+h)}\left(\gamma_{s}^{j}(\tau)\right)-\max _{s} \operatorname{Energy}_{g(\tau)}\left(\gamma_{s}^{j}(\tau)\right)
$$

$$
\leq\left[-4 \pi+\tilde{C} \epsilon+\frac{3}{4(\tau+C)} \max _{s} \operatorname{Energy}_{g(\tau)}\left(\gamma_{s}^{j}(\tau)\right)\right] h+\tilde{C} h^{2} .
$$

To see why (24.15) implies (24.4), we use the definition of the width to get

$$
W(g(\tau+h)) \leq \max _{s \in[0,1]} \operatorname{Area}_{g(\tau+h)}\left(\gamma_{s}^{j}(\tau)\right),
$$

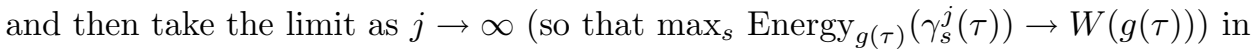
(24.15) to get

$$
\frac{W(g(\tau+h))-W(g(\tau))}{h} \leq-4 \pi+\tilde{C} \epsilon+\frac{3}{4(\tau+C)} W(g(\tau))+\tilde{C} h .
$$

Taking $\epsilon \rightarrow 0$ in (24.17) gives (24.4).

It remains to prove (24.15). First, let $\delta>0$ and $J$, depending on $\epsilon$ (and on $\tau$ ), be given by (24.12)-(24.14). If $j>J$ and

$$
\operatorname{Energy}_{g(\tau)}\left(\gamma_{s}^{j}(\tau)\right)>W(g)-\delta,
$$

then let $\cup_{i} \Sigma_{s, i}^{j}(\tau)$ be the collection of minimal spheres in (24.14). Combining (24.5), (24.14) with $F=\operatorname{Ric}_{M}$, and Lemma 24.2 gives

$$
\begin{aligned}
\frac{d}{d t} \operatorname{Area}_{t=\tau}\left(\gamma_{s}^{j}(\tau)\right) & \leq \frac{d}{d t} \operatorname{Area}_{t=\tau}\left(\cup_{i} \Sigma_{s, i}^{j}(\tau)\right)+\tilde{C} \epsilon\left\|\operatorname{Ric}_{M}\right\|_{C^{1}} \operatorname{Area}_{g(t)}\left(\gamma_{s}^{j}(\tau)\right) \\
& \leq-4 \pi-\frac{\operatorname{Energy}_{g(\tau)}\left(\gamma_{s}^{j}(\tau)\right)}{2} \min _{M} R(\tau)+\tilde{C} \epsilon \\
& \leq-4 \pi+\frac{3}{4(\tau+C)} \max _{s} \operatorname{Energy}_{g(\tau)}\left(\gamma_{s}^{j}(\tau)\right)+\tilde{C} \epsilon,
\end{aligned}
$$


where the last inequality used the lower bound (7.3) for $R(\tau)$. Since the metrics $g(t)$ vary smoothly and every sweep-out $\gamma^{j}$ has uniformly bounded energy, it is easy to see that

$$
\operatorname{Energy~}_{g(\tau+h)}\left(\gamma_{s}^{j}(\tau)\right)
$$

is a smooth function of $h$ with a uniform $C^{2}$ bound independent of both $j$ and $s$ near $h=0$ (cf. (24.5)). In particular, (24.19) and Taylor expansion gives $\bar{h}>0$ (independent of $j$ ) so that (24.15) holds for $s$ with

$$
\operatorname{Energy~}_{g(\tau)}\left(\gamma_{s}^{j}(\tau)\right)>W(g)-\delta .
$$

In the remaining case, we have

$$
\operatorname{Energy}\left(\gamma_{s}^{j}(\tau)\right) \leq W(g)-\delta
$$

so the continuity of $g(t)$ implies that (24.15) automatically holds after possibly shrinking $\bar{h}>0$.

Finally, we claim that (24.4) implies finite extinction time. Namely, rewriting $(24.4)$ as $\frac{d}{d t}\left(W(g(t))(t+C)^{-3 / 4}\right) \leq-4 \pi(t+C)^{-3 / 4}$ and integrating gives

$$
(T+C)^{-3 / 4} W(g(T)) \leq C^{-3 / 4} W(g(0))-16 \pi\left[(T+C)^{1 / 4}-C^{1 / 4}\right] .
$$

Since $W \geq 0$ by definition and the right hand side of (24.23) would become negative for $T$ sufficiently large, the theorem follows.

\section{Part 5. Growth of harmonic functions}

We next discuss some global results for harmonic functions and a few applications of function theory. We will focus on the function theory of manifolds with non-negative Ricci curvature, with the exception of Section 28 where we discuss two estimates related to nodal sets of eigenfunctions.

Recall that the classical Liouville theorem states that any bounded (or even just positive) harmonic function is constant on Euclidean space. In fact, the Euclidean gradient estimate shows that a nonconstant harmonic function must grow at least linearly. Since the partial derivatives of a Euclidean harmonic function are again harmonic, iterating this gives that on Euclidean space any harmonic function of polynomial growth is a harmonic polynomial. In particular, the dimensions of these spaces are finite on Euclidean space.

The picture gets quite a bit more complicated when we look at more general manifolds. For example, one can prescribe asymptotic values at infinity on hyperbolic space (cf. [An3]), so that even the space of bounded harmonic functions is infinite dimensional in this case.

However, [CM14] proved that each space of harmonic functions of polynomial growth is finite dimensional for manifolds with non-negative Ricci curvature. (This had been conjectured by S.T. Yau; see [Ya1], [Ya2]. The case of surfaces was settled in $[\mathbf{L i T a 2}]$.). An interesting feature of $[\mathbf{C M 1 4}]$ was that only two properties were used: a volume doubling and a Neumann Poincaré inequality, cf. 8.2.

Given an open manifold $M$ and $d>0$, we define the spaces of harmonic functions of polynomial growth of order at most $d, \mathcal{H}_{d}(M)$, using the distance function from a fixed point $p$ : 
Definition 24.4. A function $u$ is in $\mathcal{H}_{d}(M)$ if $u$ is harmonic on $M$ and

$$
|u(x)| \leq C\left(1+\operatorname{dist}_{M}(p, x)^{d}\right),
$$

for some constant $C$ and point $p \in M$.

\section{Harmonic functions and spherical harmonics}

It is worthwhile to recall the Euclidean case using polar coordinates $(\rho, \theta)$, where $\theta \in \mathbf{S}^{k-1}$. In these coordinates, the Laplacian is

$$
\Delta_{\mathbf{R}^{k}}=\rho^{-2} \Delta_{\theta}+(k-1) \rho^{-1} \frac{\partial}{\partial \rho}+\frac{\partial^{2}}{\partial \rho^{2}} .
$$

In particular, the restriction of a homogeneous harmonic polynomial of degree $d$ to $\mathbf{S}^{k-1}$ gives an eigenfunction with eigenvalue

$$
d^{2}+(k-2) d \text {. }
$$

It is then not hard to see that understanding $\mathcal{H}_{d}\left(\mathbf{R}^{k}\right)$ is a spectral problem on the compact manifold $\mathbf{S}^{k-1}$.

A similar "cone construction" holds more generally. Given a manifold $N^{k-1}$, the cone over $N$ is the manifold $C(N)=N \times[0, \infty)$ with the metric

$$
d s_{C(N)}^{2}=d r^{2}+r^{2} d s_{N}^{2} .
$$

Usually we identify $N \times\{0\}$ and refer to this point as the vertex. A direct computation shows that the Laplacians of $N$ and $C(N)$ are related by the following simple formula at $(x, r) \in N \times(0, \infty)$ :

$$
\Delta_{C(N)} u=r^{-2} \Delta_{N} u+(k-1) r^{-1} \frac{\partial}{\partial r} u+\frac{\partial^{2}}{\partial r^{2}} u .
$$

Using (25.4), we can now reinterpret the spaces $\mathcal{H}_{d}(C(N))$ :

Lemma 25.1. If $\Delta_{N} g=-\lambda g$ on $N^{k-1}$, then $r^{p} g \in \mathcal{H}_{p}(C(N))$ where

$$
p^{2}+(k-2) p=\lambda \text {. }
$$

As a consequence of Lemma 25.1, the spectral properties of $N$ are equivalent to properties of harmonic functions of polynomial growth on $C(N)$.

When $N^{k-1}$ is a submanifold of $\mathbf{S}^{n-1} \subset \mathbf{R}^{n}$, the cone over $N$ can be isometrically embedded in $\mathbf{R}^{n}$ as

$$
C(N)=\left\{x \in \mathbf{R}^{n}|x /| x \mid \in N\right\} .
$$

Note that $C(N)$ is then invariant under dilations about the origin. We get the following simple lemma whose proof is left for the reader:

Lemma 25.2. Suppose that $N^{k-1} \subset \mathbf{S}^{n-1}$. The following are equivalent:

- $N$ is minimal.

- The Euclidean mean curvature of $N \subset \mathbf{R}^{n}$ is normal to $\mathbf{S}^{n-1} \subset \mathbf{R}^{n}$.

- The coordinate functions are eigenfunctions on $N$ with eigenvalue $k-1$.

- The cone $C(N)$ is minimal. 


\section{Manifolds with non-negative Ricci curvature}

In [Ya3], Yau extended the classical Liouville theorem to complete manifolds $M$ with non-negative Ricci curvature:

A positive (or bounded) harmonic function on $M$ must be constant.

In fact, by the gradient estimate of Cheng and Yau (Theorem 8.1), any harmonic function with sublinear growth must be constant:

Corollary 26.1. [CgYa] If $M$ is complete with Ric $_{M} \geq 0$ and $d<1$, then $\mathcal{H}_{d}(M)=\{$ Constant functions $\}$.

Since $\mathbf{R}^{n}$ has non-negative Ricci curvature and the coordinate functions are harmonic, this is obviously sharp. Therefore, when $d \geq 1$ a different approach is needed. Namely, instead of showing a Liouville theorem, the point is to control the size of the space of solutions. Over the years, there were many interesting partial results (including two proofs when $M$ is a surface with non-negative sectional curvature, $[\mathbf{L i T a 2}]$ and $[\mathbf{D F}])$. For example, in [LiTa1], Li and L.F. Tam obtained the borderline case $d=1$, showing that

$$
\operatorname{dim}\left(\mathcal{H}_{1}(M)\right) \leq n+1,
$$

for an $n$-dimensional manifold with $\operatorname{Ric}_{M} \geq 0$. This is similar in spirit to the classical comparison theorems since $\operatorname{dim}\left(\mathcal{H}_{1}\left(\mathbf{R}^{n}\right)\right)=n+1$ (the $n$ coordinate functions plus the constant functions). This corresponding rigidity theorem was proven in $[\mathbf{C h C M}]$ (see $[\mathbf{L i}]$ for the special case where $M$ is Kähler):

THEOREM 26.2. [ChCM] If $M$ is complete with Ric I $_{M} \geq 0$, then every tangent cone at infinity $M_{\infty}$ splits isometrically as

$$
M_{\infty}=N \times \mathbf{R}^{\operatorname{dim}\left(\mathcal{H}_{1}(M)\right)-1} .
$$

Hence, if $\operatorname{dim}\left(\mathcal{H}_{1}(M)\right)=n+1$, then $[\mathbf{C 1}]$ implies that $M=\mathbf{R}^{n}$.

Finally, in [CM14], the spaces of polynomial growth harmonic functions were shown to be finite dimensional:

Theorem 26.3. [CM14] If $M$ is complete with Ric $_{M} \geq 0$, then $\mathcal{H}_{d}(M)$ is finite dimensional for each $d$.

The proof of Theorem 26.3 consists of two independent steps (the first does not use harmonicity):

- Given a $2 k$-dimensional subspace $H \subset \mathcal{H}_{d}(M)$ and $h \in(0,1]$, there exists a $k$-dimensional subspace $K \subset H$ and $R>0$ so that

$$
\sup _{v \in K \backslash\{0\}} \frac{\int_{B_{(1+h)^{2} R}} v^{2}}{\int_{B_{R}} v^{2}} \leq C_{1}(1+h)^{8 d} .
$$

- We bound the dimension of a subspace $K$ of harmonic functions satisfying (26.3) in terms of $h$ and $d$.

To give some feel for the argument, we will sketch a proof of the second step.

Proof. (Sketch of second step) For simplicity, suppose that $R=1$ and $h=1$. Fix a scale $r \in(0,1)$ to be chosen small. We will use two properties of manifolds with $\operatorname{Ric}_{M} \geq 0$ : 
First, we can find $N \leq C_{n} r^{-n}$ balls $B_{r}\left(x_{i}\right)$ with

$$
\chi_{B_{1}} \leq \sum_{i} \chi_{B_{r}\left(x_{i}\right)} \leq C_{n} \chi_{B_{2}}
$$

where $\chi_{E}$ is the characteristic function of a set $E$. (To do this, choose a maximal disjoint collection of balls of radius $r / 2$ and then use the volume comparison to get the second inequality in (26.4) and bound $N$.)

Second, there is a uniform Neumann Poincaré inequality: If $\int_{B_{s}(x)} f=0$, then

$$
\int_{B_{s}(x)} f^{2} \leq C_{N} s^{2} \int_{B_{s}(x)}|\nabla f|^{2} .
$$

To bound the dimension of $K$, we will construct a linear map $\mathcal{M}: K \rightarrow \mathbf{R}^{N}$ and show that $\mathcal{M}$ is injective for $r>0$ sufficiently small. We define $\mathcal{M}$ by

$$
\mathcal{M}(v)=\left(\int_{B_{r}\left(x_{1}\right)} v, \cdots, \int_{B_{r}\left(x_{N}\right)} v\right) .
$$

We will deduce a contradiction if $v \in K \backslash\{0\}$ is in the kernel of $\mathcal{M}$. In particular, (26.5) gives that for each $i$

$$
\int_{B_{r}\left(x_{i}\right)} v^{2} \leq C_{N} r^{2} \int_{B_{r}\left(x_{i}\right)}|\nabla v|^{2} .
$$

Combining this with (26.4) gives

$$
\int_{B_{1}} v^{2} \leq \sum_{i=1}^{N} \int_{B_{r}\left(x_{i}\right)} v^{2} \leq C_{N} r^{2} \sum_{i=1}^{N} \int_{B_{r}\left(x_{i}\right)}|\nabla v|^{2} \leq C_{n} C_{N} r^{2} \int_{B_{2}}|\nabla v|^{2} .
$$

We now (for the only time) use that $v$ is harmonic. Namely, the Caccioppoli inequality (or reverse Poincaré inequality) for harmonic functions gives

$$
\int_{B_{2}}|\nabla v|^{2} \leq \int_{B_{4}} v^{2}
$$

Combining (26.8) and (26.9), we get

$$
\int_{B_{1}} v^{2} \leq C_{n} C_{N} r^{2} \int_{B_{4}} v^{2}
$$

This contradicts (26.3) if $r$ is sufficiently small, completing the proof.

On Euclidean space $\mathbf{R}^{n}$, the spaces $\mathcal{H}_{d}$ are given by harmonic polynomials of degree at most $d$. In particular, it is not hard to see that

$$
\operatorname{dim}\left(\mathcal{H}_{d}\left(\mathbf{R}^{n}\right)\right) \approx C d^{n-1} .
$$

Using the correspondence between harmonic polynomials and eigenfunctions on $\mathbf{S}^{n-1}$ (see Lemma 25.1), this is closely related to Weyl's asymptotic formula on $\mathbf{S}^{n-1}$. In [CM15], the authors proved a similar sharp polynomial bound for manifolds with non-negative Ricci curvature:

THEOREM 26.4. [CM15] If $M^{n}$ is complete with Ric $_{M} \geq 0$ and $d \geq 1$, then

$$
\operatorname{dim}\left(\mathcal{H}_{d}(M)\right) \leq C d^{n-1} .
$$


Taking $M=\mathbf{R}^{n}$, (26.11) illustrates that the exponent $n-1$ is sharp in (26.12). However, as in Weyl's asymptotic formula, the constant in front of $d^{n-1}$ can be related to the volume. Namely, we actually showed the stronger statement

$$
\operatorname{dim}\left(\mathcal{H}_{d}(M)\right) \leq C_{n} \mathrm{~V}_{M} d^{n-1}+o\left(d^{n-1}\right),
$$

where

- $C_{n}$ depends only on the dimension $n$.

- $\mathrm{V}_{M}$ is the "asymptotic volume ratio" $\lim _{r \rightarrow \infty} \operatorname{Vol}\left(B_{r}\right) / r^{n}$.

- $o\left(d^{n-1}\right)$ is a function of $d$ with $\lim _{d \rightarrow \infty} o\left(d^{n-1}\right) / d^{n-1}=0$.

As noted above, Theorem 26.4 also gives lower bounds for eigenvalues on a manifold $N^{n-1}$ with $\operatorname{Ric}_{N} \geq(n-2)=\operatorname{Ric}_{\mathbf{S}^{n-1}}$. Using the sharper estimate (26.13) introduces the volume of $N$ into these eigenvalue estimates (as predicted by Weyl's asymptotic formula).

An interesting feature of these dimension estimates is that they follow from "rough" properties of $M$ and are therefore surprisingly stable under perturbation. For instance, in [CM14], we actually proved Theorem 26.3 for manifolds with a volume doubling and a Neumann Poincaré inequality; unlike a Ricci curvature bound, these properties are stable under bi-Lipschitz transformations.

This finite dimensionality was not previously known even for manifolds biLipschitz to $\mathbf{R}^{n}$ (except under additional hypotheses, cf. results of Avellenada-Lin, [AvLn], and Moser-Struwe, [MrSt]).

There are two particularly interesting directions which have not been adequately explored. The first is to develop machinery to produce harmonic functions of polynomial growth.

QUESTION 8. Suppose that $M^{n}$ has non-negative Ricci curvature and Euclidean volume growth. When can we produce harmonic functions of polynomial growth on $M$ ? The most interesting would be to solve a "Dirichlet problem at infinity," where polynomially growing harmonic functions on a tangent cone at infinity give rise to harmonic functions on $M$. In complete generality, this is likely to be rather delicate since these tangent cones need not be unique.

The second direction is to get sharper dimension estimates for holomorphic functions of polynomial growth.

QUESTION 9. If $M^{n}$ is Kähler, then each holomorphic function is harmonic so Theorem 26.4 bounds the dimension of the space of polynomial growth holomorphic functions by $C d^{n-1}$. However, on $\mathbf{C}^{n / 2}$, one get only $C d^{n / 2}$ holomorphic functions of degree d. Does the sharper bound $C d^{n / 2}$ hold? A stronger curvature condition may be necessary (cf. [Ni] for one such result).

This is just a very brief overview (omitting many interesting results), but we hope that it gives something of the flavor of the subject; the interested reader may consult $[\mathbf{C M 1 3}]$ and references therein for more information.

\section{Minimal surfaces and a generalized Bernstein theorem}

We next describe a similar finite dimensionality result for minimal submanifolds and an application of this - a "generalized Bernstein theorem" - proven in [CM16]. Recall that the Bernstein theorem implies that, through dimension seven, areaminimizing hypersurfaces are affine. A weaker form of this is true in all dimensions by the Allard regularity theorem $[\mathbf{A l}]$ : 
There exists $\delta=\delta(k, n)>0$ such that if $\Sigma^{k} \subset \mathbf{R}^{n}$ is a complete immersed minimal submanifold with

$$
\frac{\operatorname{Vol}\left(B_{r} \cap \Sigma\right)}{\operatorname{Vol}\left(B_{r} \subset \mathbf{R}^{k}\right)} \leq(1+\delta)
$$

for all $r$, then $\Sigma$ is affine.

The generalized Bernstein theorem, which should perhaps be called a generalized Allard theorem instead, shows that any upper bound on the density gives a corresponding upper bound for the dimension of the smallest affine subspace containing the minimal surface.

The results of this section apply to a large class of generalized minimal submanifolds $\Sigma^{k} \subset \mathbf{R}^{n}$ : stationary rectifiable $k$-varifolds with density at least 1 a.e. on the support. This includes the case of embedded minimal submanifolds and, for simplicity, we will focus on this case below.

Theorem 27.1. [CM16] If $\Sigma^{k} \subset \mathbf{R}^{n}$ has density bounded by $V_{\Sigma}$, then $\Sigma$ must be contained in an affine subspace of dimension at most $C_{k} V_{\Sigma}$.

Another way to think of Theorem 27.1 is that it bounds the number of linearly independent coordinate functions on $\Sigma$ in terms of its volume. The linear dependence on $\mathrm{V}_{\Sigma}$ in Theorem 27.1 is sharp; namely, any bound of the form $C_{k} \mathrm{~V}_{\Sigma}^{\alpha}$ must have $\alpha \geq 1$.

For a submanifold $\Sigma \subset \mathbf{R}^{n}$, we will define the spaces of harmonic functions of polynomial growth using the extrinsic distance; it will be clear from the context which definition we are using. Namely, given $\Sigma \subset \mathbf{R}^{n}$ and $d>0$, we define the vector spaces $\mathcal{H}_{d}(\Sigma)$ of harmonic functions of polynomial growth by:

Definition 27.2. A function $u$ is in $\mathcal{H}_{d}(\Sigma)$ if $u$ is harmonic on $\Sigma$ and

$$
|u(x)| \leq C\left(1+|x|^{d}\right),
$$

for some $C$. Thus, the coordinate functions $x_{i}$ are in $\mathcal{H}_{1}(\Sigma)$.

Since the coordinate functions are harmonic on $\Sigma$ (cf. Proposition 2.1), Theorem 27.1 follows from a bound for the dimensions of the spaces of harmonic functions on $\Sigma$ of polynomial growth:

Theorem 27.3. [CM16] Let $\Sigma^{k} \subset \mathbf{R}^{n}$ have density bounded by $V_{\Sigma}$. For any $d \geq 1$,

$$
\operatorname{dim} \mathcal{H}_{d}(\Sigma) \leq C_{k} V_{\Sigma} d^{k-1}
$$

(The spectral properties of spherical minimal submanifolds have been studied in their own right; see, for instance, Cheng-Li-Yau $[\mathbf{C g L i Y a}]$ or Choi-Wang $[\mathbf{C i W a}]$.)

27.1. Other applications of function theory. We have just seen an application of function theory to describe the geometry of the underlying space (in this case, a bound on the dimension of the space of linear growth harmonic functions controlled the complexity of the minimal submanifold). Another example is the Bochner technique and resulting topological restrictions of curvature. There are many other examples and, indeed, often these sorts of applications motivate developments in function theory. This is perhaps most evident in (one variable) complex analysis, where function theory has played a major role. 
Function theory has also played an important role in the theory of quasi-regular maps, see $[\mathbf{G}]$. Recall that a map $F: \mathbf{R}^{n} \rightarrow \mathbf{R}^{n}$ is $K$-quasi-regular if $F$ and $d F$ are in $L^{n}$ and at almost every point we have

$$
|d F| \leq K \operatorname{det}(d F) .
$$

For instance, M. Bonk and J. Heinonen used function theoretic arguments to prove:

THEOREM 27.4. [BoHj] If $M$ is a compact $n$-dimensional manifold and $F$ : $\mathbf{R}^{n} \rightarrow M$ is a (non-trivial) $K$-quasi-regular map, then the dimension of the de Rham cohomology ring of $M$ is at most $C=C(n, K)$.

Finally, we note that in the theory of quasi-regular maps, the most natural functions to study are no longer the harmonic ones. Rather, one is interested in $\mathcal{A}$-harmonic functions, i.e., functions $u$ satisfying

$$
\operatorname{div}(\mathcal{A}(\nabla u))=0,
$$

where $\mathcal{A}$ is a nonlinear map on the tangent space satisfying several natural conditions (e.g., taking $\mathcal{A}(x)=|x|^{p-2} x$ gives the so-called $p$-Laplacian).

\section{Volumes for eigensections}

Let $M^{n}$ be a closed $n$-dimensional Riemannian manifold and $V$ a vector bundle over $M$. Suppose that $\left\{f_{i}\right\}$ is an $L^{2}$-orthonormal set of eigensections of $V$ of the Laplacian with eigenvalues $\lambda_{i}$ (where $0=\lambda_{0} \leq \lambda_{1} \leq \lambda_{2} \leq \cdots$ ), that is

$$
\int_{M} f_{i} f_{j}=\delta_{i, j} \text { and } \Delta f_{i}+\lambda_{i} f_{i}=0 .
$$

Given $a=\left(a_{1}, \cdots, a_{i}\right) \in \mathbf{S}^{i-1}$, we define a function $F_{i}^{a}(\epsilon)$ by

$$
F_{i}^{a}(\epsilon)=\operatorname{Vol}\left(\left\{x \in M|| \sum_{j=1}^{i} a_{j} f_{j}(x) \mid<\epsilon\right\}\right) .
$$

Set $F_{i}(\epsilon)=F_{i}^{e_{i}}(\epsilon)$ where $e_{i}=\left(\delta_{i, j}\right)_{j}$ and define $F_{i}^{A}(\epsilon)$ by averaging $F_{i}^{a}(\epsilon)$ over $a \in \mathbf{S}^{i-1}$

$$
F_{i}^{A}(\epsilon)=f_{a \in \mathbf{S}^{i-1}} F_{i}^{a}(\epsilon)
$$

In this section we will discuss the answer to the following question of S.T. Yau:

Let $M^{n}$ be closed and $V=\Omega^{1}(M)$ the bundle of one forms on $M$. Then $\limsup _{i \rightarrow \infty} F_{i}(\epsilon)$ and $\liminf _{i \rightarrow \infty} F_{i}(\epsilon)$ are interesting functions of $\epsilon$. Are they positive? Can one estimate the behavior of $\epsilon^{-n} \liminf _{i \rightarrow \infty} F_{i}(\epsilon)$ as $\epsilon \rightarrow 0$ ? The problem may be easier if we replace $F_{i}(\epsilon)$ by $F_{i}^{A}(\epsilon)$. We can of course consider problems for $p$-forms with $p>1$.

It turns out that on a flat square torus $\liminf _{i \rightarrow \infty} F_{i}=0$ on $\Omega^{1}$. However as the next theorem shows then for eigenfunctions on any manifold $F_{i}$ is positive. We will also see below in Theorem 28.2 that the average $F_{i}^{A}$ for $p$-forms is positive and we give a sharp lower bound.

TheOrem 28.1. [CM23] Let $M^{n}$ be closed with

$$
\operatorname{Ric}_{M} \geq-(n-1) \text {. }
$$


There exists $C=C(n)>0$ and $\Lambda=\Lambda(n)>0$ such that if $f$ is a eigenfunction of $\Delta$ on $M$ with eigenvalue $\lambda \geq \Lambda$ and $0<\epsilon \leq 1$, then

$$
\operatorname{Vol}\left(\left\{\left.x \in M|| f\right|^{2}<\epsilon^{2} f_{M}|f|^{2}\right\}\right) \geq C \epsilon^{n} \operatorname{Vol}(M) .
$$

It is possible to generalize Theorem 28.1 to the case where $M$ is assumed to have the doubling property and satisfy the Neumann Poincaré inequality for $r \leq 1$. In this case, however, the exponent in $\epsilon$ may not be $n$ but rather will depend on the doubling constant $C_{D}$ and the constant $C_{p}$ in the Poincaré inequality.

In contrast to $F_{i}$ for eigenforms, then the next theorem shows that the average $F_{i}^{A}$ has always a positive lower bound.

TheOREM 28.2. [CM23] There exists $C=C(q)$ so that if $M^{n}$ is closed and $V^{q}$ is a rank $q$ vector bundle over $M$ with Laplace-type operator $\Delta_{V}$, then for $0<\epsilon \leq 1$ and $i>q$

$$
F_{i}^{A}(\epsilon) \geq C \epsilon^{q}[\operatorname{Vol}(M)]^{1+q / 2} .
$$

In contrast to eigenfunctions, $F_{i}(\epsilon)$ need not be positive for eigenforms:

Let $T^{2}$ be a flat square torus with side lengths $2 \pi$ and define one forms by

$$
\sigma_{m}=\cos \left(m x_{1}\right) d x_{1}+\sin \left(m x_{1}\right) d x_{2},
$$

then for all $m$

$$
\left\{x \in T^{2}|| \sigma_{m} \mid<1\right\}=\emptyset \text { and hence } \liminf _{i \rightarrow \infty} F_{i}(\epsilon)=0 \text { for } \epsilon<1 /\left(4 \pi^{2}\right) .
$$

\section{References}

[Al] W. Allard, On the first variation of a varifold, Ann. of Math. (2) 95 (1972) 417-491.

[Am1] F. J. Almgren, Jr., Some interior regularity theorems for minimal surfaces and an extension of Bernstein's theorem, Ann. of Math. (2) 84 (1966) 277-292.

[Am2] F. J. Almgren, Jr., Q-valued functions minimizing Dirichlet's integral and the regularity of area minimizing rectifiable currents up to codimension two, preprint.

[An1] M. T. Anderson, Ricci curvature bounds and Einstein metrics on compact manifolds, J. Amer. Math. Soc. 2 (1989) 455-490.

[An2] M. T. Anderson, Convergence and rigidity of manifolds under Ricci curvature bounds, Invent. Math. 102 (1990) 429-445.

[An3] M. T. Anderson, The Dirichlet problem at infinity for manifolds of negative curvature, J. Diff. Geom. 18 (1983) 701-721.

[AvLn] M. Avellanada and F.H. Lin, Un theoreme de Liouville pour des equations elliptiques a coefficients periodiques, C. R. Acad. Sci. Paris 309 (1989) 245-250.

[Be] S. Bernstein, Über ein geometrisches Theorem und seine Anwendung auf die partiellen Differentialgleichungen vom ellipschen Typos. Math. Zeit. 26 (1927) 551-558 (translation of the original version in Comm. Soc. Math. Kharkov 2-ème sér. 15 (1915-17) 38-45).

[Ber] L. Bers, Isolated singularities of minimal surfaces, Ann. of Math. (2) 53 (1951) 364-386.

[BDGG] E. Bombieri, E. De Giorgi, and E. Giusti, Minimal cones and the Bernstein problem, Invent. Math. 7 (1969) 243-268.

[BDM] E. Bombieri, E. De Giorgi, and M. Miranda, Una maggiorazione a priori relativa alla ipersuperfici minimali non parametriche, Arch. Rational Mech. Anal., 32 (1969) 255-267.

[Bc] S. Bochner, Curvature and Betti numbers, I, Ann. of Math. (2) 49 (1948) 379-390.

[BoHj] M. Bonk and J. Heinonen, Quasi-regular maps and cohomology, Acta Math. 86 (2001) 219-238.

[Bu] P. Buser, A note on the isoperimetric constant, Ann. Scient. Ec. Norm. 15 (1982) 213-230.

[Ca] E. Calabi, An extension of E. Hopf's maximum principle with an application to Riemannian geometry, Duke Math. Jour. 25 (1958) 45-56.

[ChC1] J. Cheeger and T.H. Colding, On the Structure of Spaces with Ricci Curvature Bounded Below; I, J. Diff. Geom. 46 (1997) 406-480. 
[ChC2] J. Cheeger and T.H. Colding, On the structure of spaces with Ricci curvature bounded below. II, J. Differential Geom. 54 (2000), no. 1, 13-35.

[ChC3] J. Cheeger and T.H. Colding, On the structure of spaces with Ricci curvature bounded below. III, J. Differential Geom. 54 (2000), no. 1, 37-74.

[ChC4] J. Cheeger and T.H. Colding, Lower bounds on Ricci curvature and the almost rigidity of warped products. Ann. of Math. (2) 144 (1996), no. 1, 189-237.

$[\mathrm{ChCM}]$ J. Cheeger, T.H. Colding, and W.P. Minicozzi II, Linear growth harmonic functions on complete manifolds with nonnegative Ricci curvature, Geom. Funct. Anal. 5 (1995), no. 6, 948-954.

[ChCTi] J. Cheeger, T.H. Colding, and G. Tian, On the singularities of spaces with bounded Ricci curvature, Geom. Funct. Anal. 12 (2002), no. 5, 873-914.

[CgLiYa] S.Y. Cheng, P. Li, and S.T. Yau, Heat equations on minimal submanifolds and their applications, Amer. J. Math. 106 (1984) 1033-1065.

[CgYa] S.Y. Cheng and S.T. Yau, Differential equations on Riemannian manifolds and their geometric applications, Comm. Pure Appl. Math. 28 (1975) 333-354.

[CiSc] H.I. Choi and R. Schoen, The space of minimal embeddings of a surface into a threedimensional manifold of positive Ricci curvature, Invent. Math. 81 (1985) 387-394.

[CiWa] H.I. Choi and A.N. Wang, A first eigenvalue estimate for minimal hypersurfaces, J. Differential Geom. 18 (1983) 559-562.

[C1] T.H. Colding, Ricci curvature and volume convergence. Ann. of Math. (2) 145 (1997), no. 3, $477-501$.

[C2] T.H. Colding, Spaces with Ricci curvature bounds. Proceedings of the International Congress of Mathematicians, Vol. II (Berlin, 1998). Doc. Math. 1998, Extra Vol. II, 299-308.

[CD1] T.H. Colding and C. De Lellis, The min-max construction of minimal surfaces, Surveys in differential geometry, Vol. 8, Lectures on Geometry and Topology held in honor of Calabi, Lawson, Siu, and Uhlenbeck at Harvard University, May 3-5, 2002, Sponsored by the Journal of Differential Geometry, (2003) 75-107, math.AP/0303305.

[CD2] T.H. Colding and C. De Lellis, Singular limit laminations, Morse index, and positive scalar curvature, math.DG/0208100.

[CH1] T.H. Colding and N. Hingston, Metrics without Morse index bounds, Duke Math. Jour., vol. 119, issue 2. (2003) 345-365.

[CH2] T.H. Colding and N. Hingston, Geodesic laminations with closed ends on surfaces and Morse index; Kupka-Smale metrics, math.DG/0208133.

[CH3] T.H. Colding and N. Hingston, in preparation.

[CM1] T.H. Colding and W.P. Minicozzi II, Minimal surfaces, Courant Lecture Notes in Math., v. 4, 1999.

[CM2] T.H. Colding and W.P. Minicozzi II, Estimates for parametric elliptic integrands, International Mathematics Research Notices, no. 6 (2002) 291-297.

[CM3] T.H. Colding and W.P. Minicozzi II, The space of embedded minimal surfaces of fixed genus in a 3-manifold I; Estimates off the axis for disks, Ann. of Math., to appear, math.AP/0210106.

[CM4] T.H. Colding and W.P. Minicozzi II, The space of embedded minimal surfaces of fixed genus in a 3-manifold II; Multi-valued graphs in disks, Ann. of Math., to appear, math.AP/0210086.

[CM5] T.H. Colding and W.P. Minicozzi II, The space of embedded minimal surfaces of fixed genus in a 3-manifold III; Planar domains, Ann. of Math., to appear, math.AP/0210141.

[CM6] T.H. Colding and W.P. Minicozzi II, The space of embedded minimal surfaces of fixed genus in a 3-manifold IV; Locally simply connected, Ann. of Math., to appear, math.AP/0210119.

[CM7] T.H. Colding and W.P. Minicozzi II, The space of embedded minimal surfaces of fixed genus in a 3-manifold $V$; Fixed genus, in preparation.

[CM8] T.H. Colding and W.P. Minicozzi II, Multi-valued minimal graphs and properness of disks, International Mathematics Research Notices, no. 21 (2002) 1111-1127.

[CM9] T.H. Colding and W.P. Minicozzi II, On the structure of embedded minimal annuli, International Mathematics Research Notices, no. 29 (2002) 1539-1552.

[CM10] T.H. Colding and W.P. Minicozzi II, Minimal annuli with and without slits, Jour. of Symplectic Geometry, vol. 1, issue 1 (2001) 47-61.

[CM11] T.H. Colding and W.P. Minicozzi II, Harmonic functions with polynomial growth, J. Diff. Geom., v. 46, no. 1 (1997) 1-77. 
[CM12] T.H. Colding and W.P. Minicozzi II, Sharp estimates for mean curvature flow of graphs, Crelle's Journal, to appear, math.AP/0305099.

[CM13] T.H. Colding and W.P. Minicozzi II, Generalized Liouville properties of manifolds, Math. Res. Lett., 3 (1996) 723-729.

[CM14] T.H. Colding and W.P. Minicozzi II, Harmonic functions on manifolds, Ann. of Math. (2), 146, no. 3 (1997) 725-747.

[CM15] T.H. Colding and W.P. Minicozzi II, Weyl type bounds for harmonic functions, Inventiones Math., 131 (1998) 257-298.

[CM16] T.H. Colding and W.P. Minicozzi II, Liouville theorems for harmonic sections and applications, Comm. Pure Appl. Math., 52 (1998) 113-138.

[CM17] T.H. Colding and W.P. Minicozzi II, Disks that are double spiral staircases, Notices of the AMS, Vol. 50, no. 3, March (2003) 327-339.

[CM18] T.H. Colding and W.P. Minicozzi II, Embedded minimal disks: Proper versus nonproper - global versus local, Transactions of the AMS, to appear. math.DG/0210328.

[CM19] T.H. Colding and W.P. Minicozzi II, Estimates for the extinction time for the Ricci flow on certain 3-manifolds and a question of Perelman, math.AP/0308090.

[CM20] T.H. Colding and W.P. Minicozzi II, Examples of embedded minimal tori without area bounds, International Mathematics Research Notices, 99 no. 20 (1999) 1097-1100.

[CM21] T.H. Colding and W.P. Minicozzi II, Complete properly embedded minimal surfaces in $\mathbf{R}^{3}$, Duke Math. J. 107 (2001) 421-426.

[CM22] T.H. Colding and W.P. Minicozzi II, Embedded minimal disks, Minimal surfaces (MSRI, 2001), ed. D. Hoffman, Clay Mathematics Proceedings, AMS, Providence (2004), 405-438, math.DG/0206146.

[CM23] T.H. Colding and W.P. Minicozzi II, Volumes for eigensections, Geom. Ded. 102 (2003) 19-24.

[Co] P. Collin, Topologie et courbure des surfaces minimales proprement plonges de $\mathbf{R}^{3}$, Ann. of Math. (2) 145 (1997) 1-31.

[DaKeTo] G. David, C. Kenig, and T. Toro, Asymptotically optimally doubling measures and Reifenberg flat sets with vanishing constant. Comm. Pure Appl. Math. 54 (2001), no. 4, 385449.

[DaSe] G. David and S. Semmes, Uniform rectifiability and quasiminimizing sets of arbitrary codimension, Mem. Amer. Math. Soc. 144 (2000), no. 687.

[DaTo] G. David, and T. Toro, Reifenberg flat metric spaces, snowballs, and embeddings. Math. Ann. 315 (1999), no. 4, 641-710.

[DG] E. De Giorgi, Frontiere orientate di misura minima, Sem. Mat. Scuola Norm. Sup. Pisa (1961) 1-56.

[De] B. Dean, Compact Embedded Minimal Surfaces of Positive Genus Without Area Bounds, Geom. Ded. 102 (2003), 45-52.

[DF] H. Donnelly and C. Fefferman, Nodal domains and growth of harmonic functions on noncompact manifolds, J. Geom. Anal. 2 (1992) 79-93.

[Do] R.T. Dong, Nodal sets of eigenfunctions on Riemann surfaces, J. Diff. Geom., 36 (1992) 493-506.

[E1] K. Ecker, Lectures on regularity for mean curvature flow, preprint.

[E2] K. Ecker, A local monotonicity formula for mean curvature flow, Ann. of Math. (2), 154 (2001) 503-525.

[EH1] K. Ecker and G. Huisken, Mean curvature evolution of entire graphs, Ann. of Math. (2), 130 (1989) 453-471.

[EH2] K. Ecker and G. Huisken, Interior estimates for hypersurfaces moving by mean curvature, Invent. Math., 105 (1991) 547-569.

[EvSp] L.C. Evans and J. Spruck, Motion of level sets by mean curvature I, J. Diff. Geom., 33 (1991) 635-681.

[Fe] H. Federer, Geometric measure theory, Springer-Verlag, Berlin-Heidelberg-New York, 1969.

[Fi] R. Finn, On equations of minimal surface type, Ann. of Math. (2), 60 (1954) 397-416.

[FHS] M.H. Freedman, J. Hass, and P. Scott, Least area incompressible surfaces in 3-manifolds, Invent. Math. 71 (1983), no. 3, 609-642.

[Fr] A. Fraser, On the free boundary variational problem for minimal disks, Comm. Pure Appl. Math. 53 (2000) 931-971. 
[Ga] S. Gallot, Volumes, courbure de Ricci et convergence des varits (d'aprs T. H. Colding et Cheeger-Colding). Sminaire Bourbaki. Vol. 1997/98. Astrisque No. 252, (1998), Exp. No. 835, 3, 7-32.

[GiTr] D. Gilbarg and N. Trudinger, Elliptic Partial Differential Equations of Second Order, Springer Verlag, 2nd Ed., (1983).

[Gr] A. Grigor'yan, The heat equation on noncompact Riemannian manifolds, English translation in Math. USSR Sb. 72 (1) (1992) 47-77.

[G] M. Gromov, Metric structures for Riemannian and non-Riemannian spaces (With appendices by M. Katz, P. Pansu and S. Semmes) Progress in Mathematics, 152. Birkhuser Boston, Inc., Boston, MA (1999).

[GLPa] M. Gromov, J. Lafontaine, and P. Pansu, Structures métriques pour les variétés riemanniennes, Cedid/Fernand Nathan, Paris (1981).

[GuSp] R. Gulliver and J. Spruck, On embedded minimal surfaces, Ann. of Math. (2) 103 (1976) 331-347; Ann. of Math. (2) 109 (1979) 407-412.

[HzKo] P. Hajlasz and P. Koskela, Sobolev meets Poincare, C. R. Acad. Sci. Paris S. I Math. 320 (1995), no. 10, 1211-1215.

[HnLn] Q. Han and F.H. Lin, Elliptic Partial Differential Equations, Courant Lecture Notes in Math., v. 1, 1997.

[Ha1] R. Hamilton, A matrix Harnack estimate for the heat equation, Comm. Anal. Geom., 1 (1993) 113-126.

[Ha2] R. Hamilton, Three-manifolds with positive Ricci curvature, J. Diff. Geom. 17 (1982) 255306.

[Ha3] R. Hamilton, The formation of singularities in the Ricci flow, Surveys in differential geometry, Vol. II (Cambridge, MA, 1993), International Press, Cambridge, MA, 1995, 7-136.

[HaNoRu] J. Hass, P. Norbury, and J.H. Rubinstein, Minimal spheres of arbitrary high Morse index, Comm. Anal. Geometry, to appear.

[Hj] J. Heinonen, Lectures on analysis on metric spaces, Springer-Verlag (2001).

[He] E. Heinz, Über die Lösungen der Minimalflächengleichung, Nachr. Akad. Wiss. Göttingen Math.-Phys. Kl, II (1952) 51-56.

[HoK] D. Hoffman and H. Karcher, Complete embedded minimal surfaces with finite total curvature, Geometry V (R. Osserman, ed.) Encyclopaedia Math. Sci. 90, Springer-Verlag, New York (1997) 5-93.

[HoMe] D. Hoffman and W. Meeks III, A complete embedded minimal surface in $\mathbf{R}^{3}$ with genus one and three ends, J. Diff. Geom. 21 (1985) 109-127.

[HoWeWo] D. Hoffman, M. Weber, and M. Wolf, An embedded genus-one helicoid, math.DG/0401080.

[H] G. Huisken, Asymptotic behavior for singularities of the mean curvature flow, J. Diff. Geom., 31 (1990) 285-299.

[I] T. Ilmanen, Elliptic regularization and partial regularity for motion by mean curvature, Mem. Amer. Math. Soc. 108 (1994), no. 520.

[Je] D. Jerison, The Poincaré inequality for vector fields satisfying Hörmander's condition, Duke Math. J. 53 (1986) 503-523.

[JXa] L. Jorge and F. Xavier, A complete minimal surface in $\mathbf{R}^{3}$ between two parallel planes, Ann. of Math. (2) 112 (1980) 203-206.

[Jo] J. Jost, Two-dimensional geometric variational problems, J. Wiley and Sons, Chichester, N.Y. (1991).

[Ka] N. Kapouleas, Complete embedded minimal surfaces of finite total curvature, J. Diff. Geom., 47 (1997) 95-169.

[K] N. Korevaar, An easy proof of the interior gradient bound for solutions to the prescribed mean curvature equation, Nonlinear functional analysis and its applications, Part 2, Proc. of Symposia in Pure Math. 45 (1986) 81-89.

[Li] P. Li, Linear growth harmonic functions on Kähler manifolds with non-negative Ricci curvature, Math. Res. Lett. 2 (1995) 79-94.

[LiTa1] P. Li and L.F. Tam, Linear growth harmonic functions on a complete manifold, J. Diff. Geom. 29 (1989) 421-425.

[LiTa2] P. Li and L.F. Tam, Complete surfaces with finite total curvature, J. Diff. Geom. 33 (1991) $139-168$. 
[LiYa] P. Li and S.T. Yau, On the parabolic kernel of the Schrödinger operator, Acta Math. 156 (1986) 153-201.

[Lc] A. Lichnerowicz, Geometrie des Groupes des Transformationes, Dunod, Paris (1958).

[Ln] F.H. Lin, Gradient estimates and blow-up analysis for stationary harmonic maps, Ann. of Math. (2) 149 (1999), no. 3, 785-829.

[Me1] W. Meeks III, The regularity of the singular set in the Colding and Minicozzi lamination theorem, Duke Math. Jour., to appear.

[Me2] W. Meeks III, The lamination metric for the Colding-Minicozzi minimal lamination, preprint.

$[\mathrm{MeP}]$ W. Meeks III and J. Perez, Conformal properties in classical minimal surface theory, preprint.

[MePRs1] W. Meeks III, J. Perez, and A. Ros, The geometry of minimal surfaces of finite genus I; Curvature estimates and quasiperiodicity, preprint.

[MePRs2] W. Meeks III, J. Perez, and A. Ros, The geometry of minimal surfaces of finite genus II; Nonexistence of one limit end examples, preprint.

[MePRs3] W. Meeks III, J. Perez, and A. Ros, The geometry of minimal surfaces of finite genus III; bounds on the topology and index of classical minimal surfaces, preprint.

[MeRo] W. Meeks III and H. Rosenberg, The uniqueness of the helicoid and the asymptotic geometry of properly embedded minimal surfaces with finite topology, preprint.

[MeSiYa] W. Meeks III, L. Simon, and S.T. Yau, Embedded minimal surfaces, exotic spheres and manifolds with positive Ricci curvature, Ann. of Math. (2) 116 (1982) 621-659.

[MeWe] W. Meeks III and M. Weber, Existence of bent helicoids and the regularity of the singular set in the Colding-Minicozzi lamination theorem, in preparation.

[MeYa1] W. Meeks III and S.T. Yau, The classical Plateau problem and the topology of three dimensional manifolds, Topology 21 (1982) 409-442.

[MeYa2] W.H. Meeks and S.T. Yau, Topology of three-dimensional manifolds and the embedding problems in minimal surface theory, Ann. of Math. (2) 112 (1980), no. 3, 441-484.

[MiMo] M.J. Micallef and J.D. Moore, Minimal two-spheres and the topology of manifolds with positive curvature on totally isotropic two-planes, Ann. of Math. (2) 127 (1988) no. 1 199-227.

[MrSt] J. Moser and M. Struwe, On a Liouville-type theorem for linear and nonlinear elliptic differential equations on a torus, Bol. Soc. Bra. Mat. 23 (1992) 1-20.

[Na] N. Nadirashvili, Hadamard's and Calabi-Yau's conjectures on negatively curved and minimal surfaces, Invent. Math. 126 (1996) 457-465.

[Ni] L. Ni, A monotonicity formula on complete Kähler manifolds with nonnegative bisectional curvature math, DG/0307275.

[Ob] M. Obata, Certain conditions for a Riemannian manifold to be isometric to a sphere, J. Math. Soc. Japan 14 (1960) 333-340.

[Os] R. Osserman, A survey of minimal surfaces, Dover, 2nd. edition (1986).

[Pe1] G. Perelman, The entropy formula for the Ricci flow and its geometric applications, math.DG/0211159.

[Pe2] G. Perelman, Ricci flow with surgery on three-manifolds, math.DG/0303109.

[Pe3] G. Perelman, Finite extinction time for the solutions to the Ricci flow on certain threemanifolds, math.DG/0307245.

[Pe4] G. Perelman, A complete Riemannian manifold of positive Ricci curvature with Euclidean volume growth and nonunique asymptotic cone. Comparison geometry (Berkeley, CA, 199394), 165-166, Math. Sci. Res. Inst. Publ., 30, Cambridge Univ. Press, Cambridge, 1997.

[Pi] J.T. Pitts, Existence and regularity of minimal surfaces on Riemannian manifolds, Princeton University Press, Princeton, N.J.; University of Tokyo Press, Tokyo (1981).

[PiRu] J.T. Pitts and J.H. Rubinstein, Applications of minmax to minimal surfaces and the topology of 3-manifolds, Miniconference on geometry and partial differential equations, Proceedings of the CMA, Australia National University (1986).

[Pr] P. Price, A monotonicity formula for Yang-Mills fields. Manuscripta Math, 43 (1983), no. 2-3, 131-166.

[Re] E.R. Reifenberg, Solution of the Plateau Problem for $m$-dimensional surfaces of varying topological type. Acta Math. 1041960 1-92.

[Ro] H. Rosenberg, Some recent developments in the theory of properly embedded minimal surfaces in $\mathbf{R}^{3}$, Seminare Bourbaki 1991/92, Asterisque No. 206 (1992) 463-535. 
[SaUh] J. Sacks and K. Uhlenbeck, The existence of minimal immersions of 2-spheres, Ann. of Math. (2) 113 (1981) no. 1, 1-24.

[SC] L. Saloff-Coste, Uniformly elliptic operators on Riemannian manifolds, J. Diff. Geom. 36 (1992) 417-450.

[Sc1] R. Schoen, Estimates for stable minimal surfaces in three-dimensional manifolds, In Seminar on Minimal Submanifolds, Ann. of Math. Studies, vol. 103, Princeton University Press, Princeton, N.J., (1983) 111-126.

[Sc2] R. Schoen, Analytic aspects of the harmonic map problem, MSRI publ. 2, Springer, New York-Berlin, (1984) 321-358.

[ScSi] R. Schoen and L. Simon, Regularity of simply connected surfaces with quasi-conformal Gauss map, In Seminar on Minimal Submanifolds, Annals of Math. Studies, vol. 103, Princeton University Press, Princeton, N.J., (1983) 127-145.

[ScSiYa] R. Schoen, L. Simon, and S.T. Yau, Curvature estimates for minimal hypersurfaces, Acta Math. 134 (1975) 275-288.

[ScUh] R. Schoen and K. Uhlenbeck, A regularity theory for harmonic maps, J. Diff. Geom. 17 (1982) 307-335.

[ScYa1] R. Schoen and S.T. Yau, Lectures on differential geometry, International Press 1994.

[ScYa2] R. Schoen and S.T. Yau, Existence of incompressible minimal surfaces and the topology of three dimensional manifolds with nonnegative scalar curvature, Ann. of Math. (2) 110 (1979) $127-142$.

[ScYa3] R. Schoen and S.T. Yau, Lectures on harmonic maps, International Press 1997.

[Se] S. Semmes, Chord-arc surfaces with small constant II: Good parameterizations, Adv. Math. 88 (1991) 170-199.

[Si1] L. Simon, Asymptotic behaviour of minimal graphs over exterior domains, Ann. Inst. H. Poincar Anal. Non Linaire 4 (1987) 231-242.

[Si2] L. Simon, Remarks on curvature estimates for minimal hypersurfaces, Duke Math. J. 43 (1976) 545-553.

[Si3] L. Simon, Singularities of Geometric Variational Problems, In Nonlinear Partial Differential Equations in Differential Geometry (R. Hardt and M. Wolf, Ed.), American Mathematical Society, Providence (1996) 185-223.

[Sim] J. Simons, Minimal varieties in Riemannian manifolds, Ann. of Math. (2) 88 (1968) 62-105.

[Sm] F. Smith, On the existence of embedded minimal 2-spheres in the 3-sphere, endowed with an arbitrary Riemannian metric, supervisor L. Simon, University of Melbourne (1982).

[St] M. Struwe, Geometric evolution problems, In Nonlinear Partial Differential Equations in Differential Geometry (R. Hardt and M. Wolf, Ed.), American Mathematical Society, Providence (1996) 259-339.

[To] T. Toro, Geometric conditions and existence of bi-Lipschitz parameterizations. Duke Math. J. 77 (1995), no. 1, 193-227.

[Tr] M. Traizet, Adding handles to Riemann's minimal surfaces, J. Inst. Math. Jussieu 1 (2002) $145-174$.

[WeWo] M. Weber and M. Wolf, Teichmüller theory and handle addition for minimal surfaces, Ann. of Math. (2), 156 (2002) 713-795.

[W] B. White, Evolution of curves and surfaces by mean curvature, Proceedings of the ICM (2002).

[Y] S.D. Yang, A connected sum construction for complete minimal surfaces of finite total curvature, Comm. Anal. Geom. 9 (2001), no. 1, 115-167.

[Ya1] S.T. Yau, Nonlinear analysis in geometry, L'Eseignement Mathematique (2) 33 (1987) 109158.

[Ya2] S.T. Yau, Open problems in geometry, Proc. Sympos. Pure Math., 54, Part 1, American Mathematical Society, Providence, 1993.

[Ya3] S.T. Yau, Harmonic functions on complete Riemannian manifolds, Comm. Pure Appl. Math. 28 (1975) 201-228.

Courant Institute of Mathematical Sciences, 251 Mercer Street, New York, Ny 10012

Department of Mathematics, Johns Hopkins University, 3400 N. Charles St., BaltiMORE, MD 21218

E-mail address: colding@cims.nyu.edu and minicozz@math.jhu.edu 